Pontifícia U Miversidade $C_{\text {atólica }}$

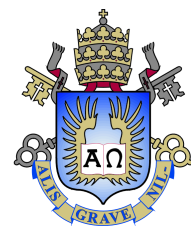

Renato Ricardo de Paula

\title{
Modelo em meios porosos em contato com reservatórios
}

Dissertação apresentada como requisito parcial para obtenção do grau de Mestre pelo Programa de Pós-graduação em Matemática do Departamento de Matemática do Centro Técnico Científico da PUC-Rio.

Orientador

Prof. Simon Richard Griffiths

Co-orientadora: Profa. Ana Patrícia Carvalho Gonçalves 


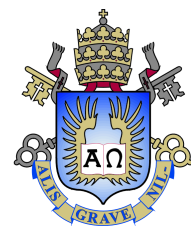

\section{Renato Ricardo de Paula}

\section{Modelo em meios porosos em contato com reservatórios}

Dissertação apresentada como requisito parcial para obtenção do grau de Mestre pelo Programa de Pós-graduação em Matemática do Departamento de Matemática do Centro Técnico Científico da PUC-Rio. Aprovada pela Comissão Examinadora abaixo assinada.

Prof. Simon Richard Griffiths

Orientador

Departamento de Matemática - PUC-Rio

Profa. Ana Patrícia Carvalho Gonçalves

Coorientadora

Instituto Superior Técnico - Universidade de Lisboa

Profa. Adriana Neumann de Oliveira Instituto de Matemática - UFRGS

Prof. Freddy Rolando Hernandez Romero Instituto de Matemática - UFF

Prof. Glauco Valle da Silva Coelho Instituto de Matemática - UFRJ

Prof. Márcio da Silveira Carvalho

Coordenador Setorial do Centro Técnico Científico - PUC-Rio 
Todos os direitos reservados. É proibida a reprodução total ou parcial do trabalho sem autorização da universidade, do autor e do orientador.

\section{Renato Ricardo de Paula}

Graduou-se em Bacharelado de Matemática com Ênfase em Matemática Computacional na Universidade Federal Fluminense (Volta Redonda).

Ficha Catalográfica

Paula, Renato Ricardo de

Modelo em meios porosos em contato com reservatórios / Renato Ricardo de Paula; orientador: Simon Richard Griffiths; co-orientadora: Ana Patrícia Carvalho Gonçalves. - 2017.

v., $77 \mathrm{f:}$ il. ; $30 \mathrm{~cm}$

Dissertação (mestrado) - Pontifícia Universidade Católica do Rio de Janeiro, Departamento de Matemática.

Inclui bibliografia

1. Processo de Exclusão;. 2. Modelo em Meios porosos;. 3. Equação em Meios porosos;. 4. Limite Hidrodinâmico;. 5. Matriz Ansatz. I. Griffiths, Simon Richard. II. Gonçalves, Ana Patrícia Carvalho. III. Pontifícia Universidade Católica do Rio de Janeiro. Departamento de Matemática. IV. Título. 


\section{Agradecimentos}

Agradeço à minha família pelo carinho e apoio em todos os momentos da minha vida.

Agradeço também os professores, funcionários e amigos do departamento de matemática da PUC-Rio.

Meu grande agradecimento aos meus orientadores Simon Griffiths e Patrícia Gonçalves por todo o apoio, incentivo e dedicação.

À Laís pelo seu carinho, apoio e companheirismo.

Ao CNPq pelo apoio financeiro. 


\section{Resumo}

Paula, Renato Ricardo de; Griffiths, Simon Richard; Gonçalves, Ana Patrícia Carvalho; . Modelo em meios porosos em contato com reservatórios. Rio de Janeiro, 2017. 77p. Dissertação de Mestrado - Departamento de Matemática, Pontifícia Universidade Católica do Rio de Janeiro.

A primeira parte da dissertação é dedicada ao estudo do modelo em meios porosos em contato com reservatórios e à obtenção, heurística, da equação hidrodinâmica para esse modelo, com o intuito de iniciar o estudo do limite hidrodinâmico que garante que a evolução da densidade de partículas desse modelo é descrita pela solução fraca da equação hidrodinâmica, nomeadamente, a equação em meios porosos com condições de Dirichlet. A segunda parte da dissertação é dedicada ao estudo do método da representação matricial, a chamada "matriz ansatz", que será utilizado para caracterizar as medidas estacionárias de sistemas de partículas fora do equilíbrio. Usaremos o processo de exclusão simples simétrico como motivação para apresentar as técnicas utilizadas nesse método. Munido dessas técnicas conseguimos obter pela primeira vez a função de correlação de segunda ordem para o processo de exclusão simples simétrico em contato com reservatórios lentos, e além disso, conseguimos obter informação do estado estacionário do modelo em meios porosos em contato com reservatórios.

\section{Palavras-chave}

Processo de Exclusão; Modelo em Meios porosos; Equação em Meios porosos; Limite Hidrodinâmico; Matriz Ansatz 


\section{Abstract}

Paula, Renato Ricardo de; Griffiths, Simon Richard (Advisor); Gonçalves, Ana Patrícia Carvalho (Co-Advisor). Porous Medium Model in contact with Reservoirs. Rio de Janeiro, 2017. 77p. Dissertação de Mestrado - Departamento de Matemática, Pontifícia Universidade Católica do Rio de Janeiro.

The first part of the dissertation is dedicated to the study of the porous medium model in contact with reservoirs and to, heuristically, obtain the hydrodynamic equation for this model, with the pursuit of starting the study of the hydrodynamic limit which guarantees that the evolution of the density of particles of this model is described by the weak solution of the hydrodynamic equation, namely, the porous medium equation with Dirichlet boundary conditions. The second part of the dissertation is dedicated to the study of the matrix representation method, the so-called "matrix ansatz", which will be used to characterize the stationary measures of particle systems out of equilibrium. For warming up, we will use the symmetric simple exclusion process as a toy model to present the techniques used in this method. With those techniques, for the first time we obtained the second order correlation function for the symmetric simple exclusion process in contact with slow reservoirs, and in addition, we were able to obtain information about the steady state of the porous medium model in contact with reservoirs.

\section{Keywords}

Exclusion Process; $\quad$ Porous Medium Model; Porous Medium Equation; Hydrodynamic Limit; Matrix Ansatz 


\section{Sumário}

1 Introdução 11

2 O modelo em meios porosos em contato com reservatórios $\quad 13$

$\begin{array}{lll}2.1 & \text { Processo de exclusão } & 13\end{array}$

$\begin{array}{ll}2.2 \text { Uma definição informal para o modelo } & 15\end{array}$

$\begin{array}{ll}2.3 \text { Construção de Harris } & 18\end{array}$

2.4 Construção via gerador infinitesimal 22

2.5 Representação matricial 24

$\begin{array}{ll}2.6 & \text { Medidas estacionárias } \\ \end{array}$

3 Heurística da equação hidrodinâmica $\quad 29$

3.1 Corrente microscópica 29

3.2 Limite Hidrodinâmico 32

$\begin{array}{lll}3.3 & \text { Equação em meios porosos } & 38\end{array}$

$4 \quad$ Estados estacionários fora do equilíbrio $\quad 42$

4.1 SSEP em contato com reservatórios $\quad 42$

4.2 Sistemas em equilíbrio e fora do equilíbrio 43

$\begin{array}{lll}5 & \text { Matriz ansatz } & 47\end{array}$

$\begin{array}{lll}5.1 & \text { A master equation } & 47\end{array}$

5.2 A matriz ansatz 48

5.3 Propriedades e exemplos das matrizes 54

$6 \quad$ Utilizando a matriz ansatz $\quad 56$

6.1 A matriz ansatz para SSEP em contato com reservatórios 57

6.2 A matriz ansatz para o PMM em contato com reservatórios 60

6.3 A matriz ansatz para o SSEP em contato com reservatórios lentos 62

$\begin{array}{ll}\text { Referências bibliográficas } & 67\end{array}$

$\begin{array}{lll}\text { A Semigrupos e geradores } & 69\end{array}$

B Martingais e Processos de Markov $\quad 72$

$\begin{array}{ll}\text { C Gerando um processo de Poisson } & 76\end{array}$ 


\section{Lista de figuras}

1.1 Um canal mantido fora do equilíbrio através da aplicação de um potencial químico. Prevê-se que será atingido um estado estacionário fora do equilíbrio, no qual as partículas fluem de um reservatório para outro a uma taxa constante.

2.1 Processo de exclusão simples.

2.2 Processo de exclusão simples simétrico. 14

2.3 Processo de exclusão simples assimétrico. 14

$\begin{array}{lll}2.4 & \text { Salto de } x \text { para } x+1 \text { com taxa } 1 . & 15\end{array}$

2.5 Salto de $x$ para $x+1$ com taxa $1 . \quad 16$

2.6 Salto de $x$ para $x+1$ com taxa $2 . \quad 16$

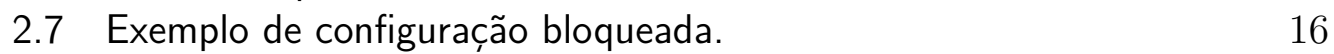

2.8 Configuração com seus possíveis saltos e taxas referentes ao PMM em contato com reservatórios.

2.9 Configuração com seus possíveis saltos e taxas referentes ao SSEP em contato com reservatórios lentos.

2.10 Representação da configuração inicial $\eta_{0}$. 19

2.11 SSEP em contato com reservatórios. 20

$\begin{array}{ll}2.12 \text { PMM em contato com reservatórios. } & 21\end{array}$

2.13 Configuração com seus possíveis saltos e taxas referentes ao PMM em contato com reservatórios.

5.1 O procedimento pelo qual uma configuração de partículas é representada por um produto de matrizes.

6.1 SSEP em contato com reservatórios. 


\section{Lista de tabelas}

2.1 Tempo em que cada relógio de Poisson toca. 
You can't be perfect, but if you don't try, you won't be good enough.

Paul Halmos. 


\section{Introdução}

O estudo do limite hidrodinâmico de sistemas de partículas dá validade a várias equações diferenciais parciais que são estudadas na literatura, e também permite provar a existência de soluções fracas de tais equações. No caso de sistemas de partículas com taxas simétricas, geralmente o estudo tem como equação hidrodinâmica equações parabólicas lineares com condições de fronteira linear, como pode ser visto em [8] e [9]. Mas no caso de equações não lineares, tal estudo se encontra em desenvolvimento, e um dos objetivos desta dissertação será estudar exatamente o modelo em meios porosos em contato com reservatórios, o qual é um sistema não linear.

O estudo do comportamento assintótico de sistemas de partículas interagentes fora do equilíbrio é um dos grandes problemas na área sistema de sistemas de partículas. Para ilustrar, considere um volume finito contendo partículas interagentes e que está acoplado a dois reservatórios de partículas, um em cada fronteira com diferentes potenciais químicos cada um. Nesta situação, prevê-se um fluxo líquido de partículas através do sistema indo do reservatório com maior potencial químico para o reservatório oposto. Após algumas transições iniciais, esperamos que se estabeleça um estado onde haja um fluxo médio (não-nulo) de partículas, que seja constante ao longo do espaço e do tempo, veja Figura 1.1. Este fluxo revela que o sistema está em um estado estacionário fora do equilíbrio, que será o estado de grande interesse dessa dissertação.

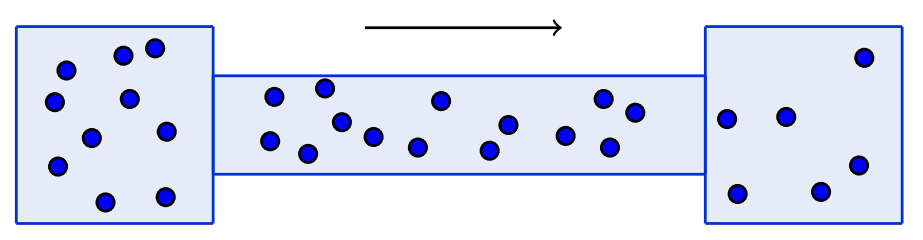

Figura 1.1: Um canal mantido fora do equilíbrio através da aplicação de um potencial químico. Prevê-se que será atingido um estado estacionário fora do equilíbrio, no qual as partículas fluem de um reservatório para outro a uma taxa constante.

Assim, podemos dizer que esta dissertação se concentra em dois grandes objetivos: 
1. Estudar o modelo em meios porosos em contato com reservatórios e apresentar, de forma heurística, a dedução da equação hidrodinâmica para o mesmo;

2. Apresentar o método da representação matricial (matriz ansatz), que será aplicado aos modelos presentes no Capítulo 6, Seções 6.1, 6.2 e 6.3 para obtermos informações a respeito do seu estado estacionário fora do equilíbrio.

Adicionalmente, vale ressaltar que na abordagem 2., toda solução do problema é reduzida a encontrar duas matrizes e dois vetores que satisfazem regras algébricas muito simples. Com isso, obtemos várias formas explícitas para estas matrizes que são, no caso geral, de dimensão infinita. Nossa abordagem permite então, encontrar expressões exatas para os perfis de corrente e densidade para vários modelos. Uma descrição do que se estuda nessa dissertação segue abaixo.

No segundo capítulo, apresentamos uma definição informal para o modelo em meios porosos em contato com reservatórios e em seguida, definimos o modelo formalmente utilizando a construção de Harris e o gerador infinitesimal do processo. Terminamos o capítulo apresentando uma representação matricial para o modelo e obtemos informação sobre sua medida estacionária no estado de equilíbrio.

No terceiro capítulo, apresentamos uma dedução heurística da equação hidrodinâmica para o modelo em meios porosos em contato com reservatórios. Começamos o capítulo mostrando que quando o número de sítios presentes no nosso sistema de partículas for grande, então a densidade de partículas se comportará como a solução da equação em meios porosos com condições de Dirichlet. A segunda parte do capítulo é dedicada a encontrar a formulação fraca para a equação em meios porosos com condições de Dirichlet e mostramos que a mesma é única. Em seguida, o quarto capítulo apresenta o processo de exclusão simples simétrico em contato com reservatórios com o objetivo de analisar o comportamento do mesmo em um estado de equilíbrio e fora do equilíbrio. Sob inspiração das técnicas usadas em $[3,5,6]$, o quinto capítulo apresenta o método da representação matricial (matriz ansatz) para caracterizar as medidas estacionárias de sistemas de partículas fora do equilíbrio.

Finalmente, o sexto e último capítulo dedica-se à aplicação do método da matriz ansatz para o processo de exclusão simples simétrico em contato com reservatórios, para o modelo em meios porosos em contato com reservatórios, e para o processo de exclusão simples simétrico em contato com reservatórios lentos. 


\section{2}

\section{O modelo em meios porosos em contato com reservatórios}

Neste capítulo faremos a descrição do modelo em meios porosos $(\mathrm{PMM})^{1}$ em contato com reservatórios. Antes de descrever tal modelo, na Seção 2.1, começaremos por descrever o processo de exclusão, uma vez que o PMM em contato com reservatórios consiste em um processo de exclusão cujas taxas de salto dependem do número de partículas na vizinhança do salto [19]. Na Seção 2.2, apresentaremos uma definição informal para o PMM em contato em reservatórios. Em seguida, na Seção 2.3, faremos a construção de Harris para o PMM em contato com reservatórios, e ilustraremos a diferença do mesmo com o processo de exclusão simples simétrico (SSEP) em contato com reservatórios. $\mathrm{Na}$ Seção 2.4, apresentaremos a construção do modelo via gerador infinitesimal, de modo que na Seção 2.5, somos capazes de representar a ação deste gerador infinitesimal matricialmente. Por fim, na Seção 2.6, mostraremos que a medida estacionária do PMM em contato com reservatórios quando $\alpha=\beta$ é a medida Bernoulli produto.

\section{1}

Processo de exclusão

Informalmente, o processo de exclusão consiste em um conjunto de partículas que ocupam um certo conjunto discreto de sítios; cada elo (ou seja, cada par de sítios) tem associado um relógio que toca aleatoriamente, de acordo com uma certa regra probabilística, e quando um relógio de um elo toca, as partículas nos sítios associados a esse elo trocam de posição, isto é, se houver no máximo uma partícula em um dos sítios correspondentes, então ela executa um salto para o sítio vazio com uma certa taxa. No entanto, se ambos os sítios estão vazios ou ambos estão ocupados, então nada acontece e os relógios reiniciam a sua contagem do tempo.

Assumimos que os relógios acima são relógios de Poisson, isto é, são processos de Poisson, e mais detalhes serão dados na Seção 2.2 quando formos fazer a descrição do modelo em meio porosos. Assim, o tempo entre os saltos associados aos elos são variáveis aleatórias exponenciais independentes, e a escolha desta lei de probabilidade para os relógios nos garante que o processo

\footnotetext{
${ }^{1}$ Esta abreviatura vem do inglês "porous medium model".
} 
satisfaz a propriedade de Markov, isto é, o futuro do processo, condicionado ao presente e passado, somente depende do presente. Devido às condições impostas na distribuição dos relógios, notamos também que dois relógios não tocam simultaneamente, e sendo assim, cada vez que um relógio toca, ou um salto é executado ou nada acontece. O processo que acabamos de descrever se chama processo de exclusão, uma vez que uma partícula só salta para um certo sítio caso este esteja vazio, no caso em que o sítio esteja ocupado, a partícula não se mexe e espera um novo toque do relógio.

Um processo de exclusão se diz simples quando o salto de uma partícula no sítio $x$ ocorre apenas para um de seus sítios vizinhos, isto é, $x-1$ ou $x+1$.

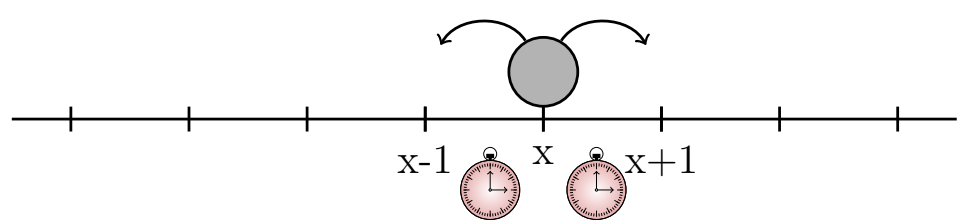

Figura 2.1: Processo de exclusão simples.

Um processo de exclusão simples é dito ser simétrico quando a taxa de salto $p>0$ é igual para todos os vizinhos, ou seja, se o processo estiver definido, por exemplo, em $\mathbb{Z}$, ele vai saltar de $x$ para $x-1$ e de $x$ para $x+1$ com a mesma taxa $p=\frac{1}{2}$.

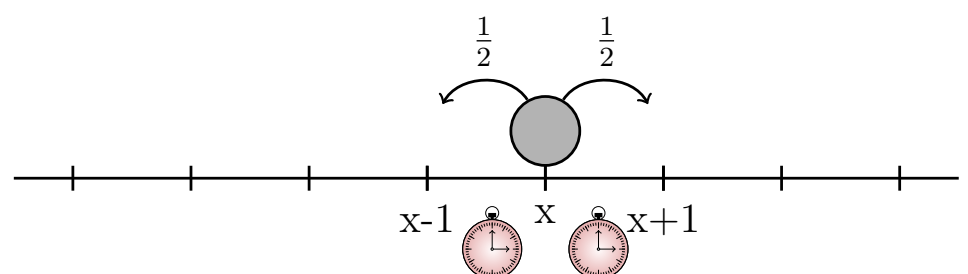

Figura 2.2: Processo de exclusão simples simétrico.

Caso em um processo de exclusão simples a partícula salte de $x$ para $x+1$ com certa taxa $p$, e de $x$ para $x-1$ com taxa $q$, com $p+q=1$, o processo se diz um processo de exclusão simples assimétrico.

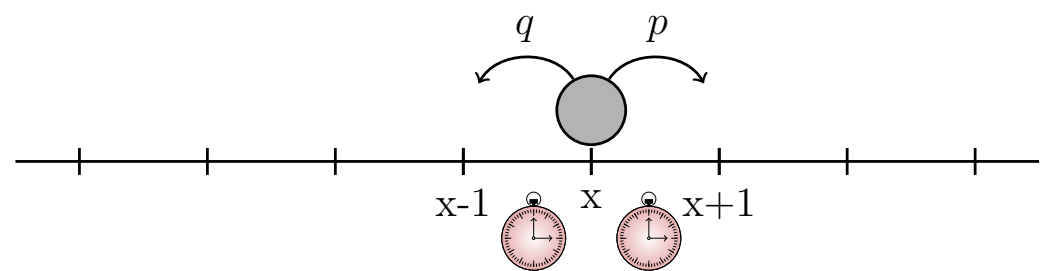

Figura 2.3: Processo de exclusão simples assimétrico. 
Munido dos conceitos de processos de exclusão apresentados anteriormente, vamos considerar agora o nosso primeiro modelo de interesse: o modelo em meios porosos, e depois vamos explicar como colocamos este modelo em contato com reservatórios de partículas.

\section{2}

\section{Uma definição informal para o modelo}

Notamos que o PMM em contato com reservatórios em $\mathbb{Z}$ foi estudado em [11]. Seja agora $N \geq 1$ e suponha que temos $N+1$ sítios, isto é, temos uma malha com os pontos $\{0,1,2, \cdots, N-1, N\}$. No interior da malha, ou seja, em $\Sigma_{N}=\{1, \cdots, N-1\}$, a que chamamos bulk, cada sítio contém no máximo uma partícula. Em cada elo $\{x, x+1\}$ da malha existe um relógio de Poisson de parâmetro 1, e relógios correspondentes a elos diferentes são independentes. O mecanismo de escolha do salto vai ocorrer da seguinte maneira: se um relógio em um elo do interior da malha toca e se nos sítios associados a esse elo há pelo menos uma partícula, esta partícula troca a sua posição nos respectivos sítios, isto é, se a partícula estiver no sítio $x$ e tiver tocado o relógio correspondente ao elo $\{x, x+1\}$, então, a partícula salta para o sítio $x+1$ caso este esteja desocupado. Caso contrário, permanece no sítio $x$ e aguarda um novo toque do relógio. No entanto, a partícula só irá saltar para o sítio $x+1$ dependendo do número de partículas nos sítios "vizinhos". Mais especificamente, uma partícula no sítio $x$ só salta para $x+1$ se houver pelo menos uma partícula no sítio $x-1$ ou no sítio $x+2$, e esse salto ocorre com uma taxa dada pela soma do número de partículas no sítio $x-1$ e o número de partículas no sítio $x+2$. Abaixo vamos fazer uma descrição dos eventos que podem ocorrer para o modelo que estamos descrevendo.

Na configuração abaixo supomos que o relógio associado ao elo $\{x, x+1\}$ toca. Como existe uma partícula no sítio $x$ e nenhuma partícula no sítio $x+1$, então, a regra de exclusão permite que o salto seja executado. Além disso, como existe uma partícula no sítio $x-1$, de acordo com a taxa de salto introduzida acima a partícula salta com taxa 1 .

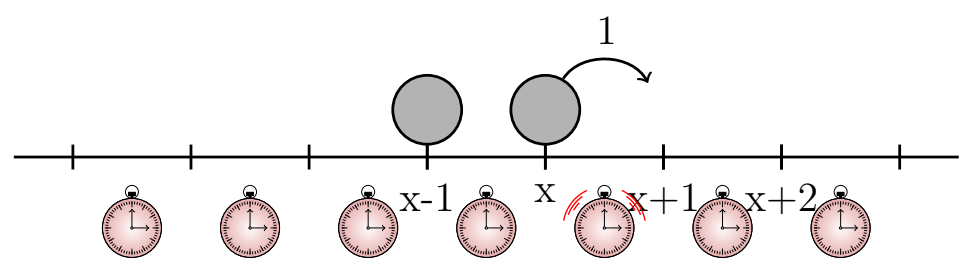

Figura 2.4: Salto de $x$ para $x+1$ com taxa 1 .

Analogamente, na configuração abaixo supomos que o relógio associado 
ao elo $\{x, x+1\}$ toca. Como existe uma partícula no sítio $x$ e nenhuma partícula no sítio $x+1$, então, a regra de exclusão permite que o salto seja executado. Além disso, com a taxa de salto introduzida acima, como existe uma partícula no sítio $x+2$ a partícula salta com taxa 1 .

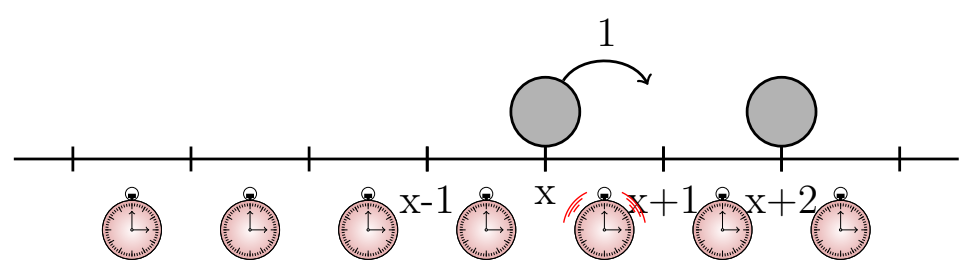

Figura 2.5: Salto de $x$ para $x+1$ com taxa 1 .

Finalmente, na figura abaixo, temos exatamente a mesma situação descrita acima, no entanto, como existe uma partícula no sítio $x-1$ e uma partícula no sítio $x+2$, a taxa do salto é 2 em vez de 1 .

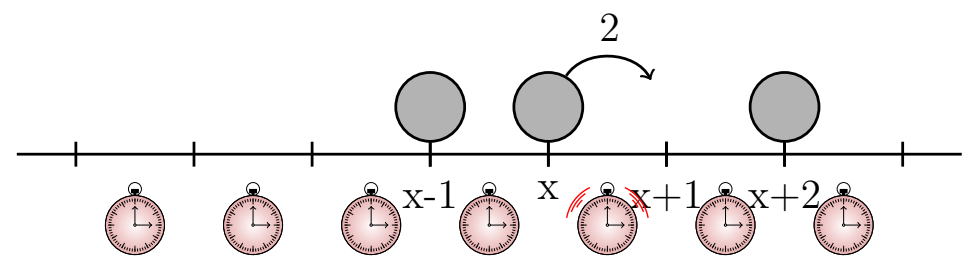

Figura 2.6: Salto de $x$ para $x+1$ com taxa 2 .

A dinâmica que pretendemos definir é simétrica, no sentido em que as taxas de salto de $x$ para $x+1$ são análogas às taxas de salto de $x$ para $x-1$. Esta escolha para as taxas dos saltos faz com que hajam configurações bloqueadas no bulk do nosso sistema. Essas configurações chamadas bloqueadas são configurações onde todo par de partículas está a uma distância maior do que dois, assim, essas configurações não podem evoluir a partir da dinâmica descrita acima.

Abaixo temos uma configuração que não evolui pela dinâmica que acabamos de descrever. Como a distância entre cada uma das partículas é igual a três, elas não se mexem devido às taxas de salto que definimos anteriormente, que exigem que o salto se dê se existir no mínimo uma partícula nos sítios vizinhos.

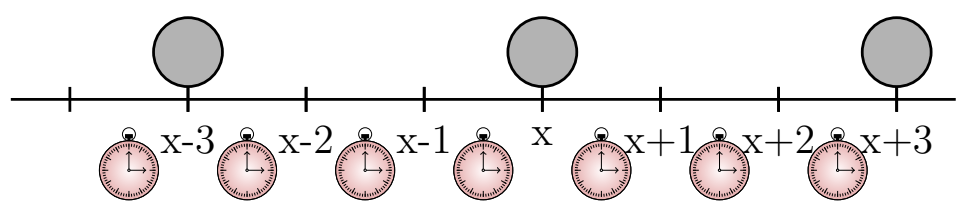

Figura 2.7: Exemplo de configuração bloqueada. 
Para terminar a descrição da dinâmica que iremos considerar, adicionaremos dois reservatórios de partículas nos sítios $x=0$ e $x=N$. Associaremos ao elo $\{0,1\}$ (respectivamente ao elo $\{N-1, N\}$ ) dois relógios de Poisson, um com taxa $\alpha$ (respectivamente com taxa $\beta$ ) associado à $\{0,1\}$ (respectivamente à $\{N, N-1\})$, e outro com taxa $1-\alpha$ (respectivamente com taxa $1-\beta$ ) associado à $\{1,0\}$ (respectivamente à $\{N-1, N\}$ ), onde $\alpha, \beta \in(0,1)$. Assim, as partículas podem entrar no bulk para o sítio 1 com taxa $\alpha$ (respectivamente para o sítio $N-1$ com taxa $\beta$ ) respeitando a regra de exclusão, e podem sair do sítio 1 com taxa $(1-\alpha)$ (respectivamente do sítio $N-1$ com taxa $(1-\beta)$ ).

O caso em que a partícula salta no bulk com taxa $\frac{1}{2}$ para o sítio da direita, e com taxa $\frac{1}{2}$ para o sítio da esquerda, é chamado de processo de exclusão simples simétrico em contato com reservatórios, e foi estudado em [13]. Este processo nos servirá de motivação no Capítulo 4 quando formos trabalhar com o método da matriz ansatz.

Abaixo é apresentado um exemplo de uma configuração com seus possíveis saltos e taxas, onde as partículas no bulk estão pintadas de cinza, e as partículas nos reservatórios estão pintadas de vermelho.

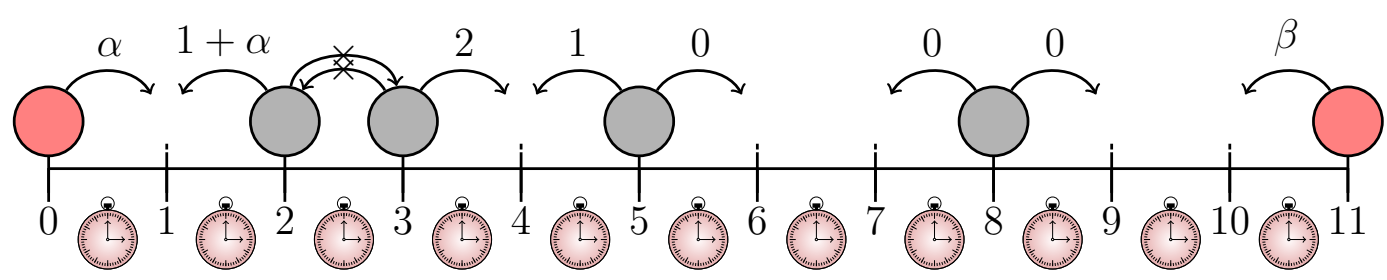

Figura 2.8: Configuração com seus possíveis saltos e taxas referentes ao PMM em contato com reservatórios.

Observação 2.1. Observe que na configuração acima temos $N=11$ mas apenas fixamos a configuração no bulk, isto é, nos sítios $\{1,2, \cdots, 10\}$, uma vez que nos sítios $x=0$ e $x=11$ a configuração não está definida.

O sistema de partículas que introduzimos nesta seção é o modelo em meios porosos colocado em contato com reservatórios de partículas com uma certa densidade, o qual chamaremos de modelos em meios porosos em contato com reservatórios.

Um outro sistema de partículas que será importante nesta dissertação é o processo de exclusão simples simétrico em contato com reservatórios lentos. No bulk este processo tem a mesma dinâmica do processo de exclusão simples simétrico, já nas fronteiras, a dinâmica é um pouco diferente do modelo que vimos acima. Para o SSEP em contato com reservatórios lentos associamos ao elo $\{0,1\}$ (respectivamente ao elo $\{N-1, N\}$ ) dois relógios de Poisson, um com 
taxa $\frac{\alpha}{N^{\theta}}$ (respectivamente com taxa $\frac{\beta}{N^{\theta}}$ ) associado à $\{0,1\}$ (respectivamente à $\{N, N-1\}$ ), e outro com taxa $\frac{1-\alpha}{N^{\theta}}$ (respectivamente com taxa $\left.\frac{1-\beta}{N^{\theta}}\right)$ associado à $\{1,0\}$ (respectivamente à $\{N-1, N\}$ ), onde $\alpha, \beta \in(0,1)$ e $\theta \geq 0$, como pode ser visto abaixo:

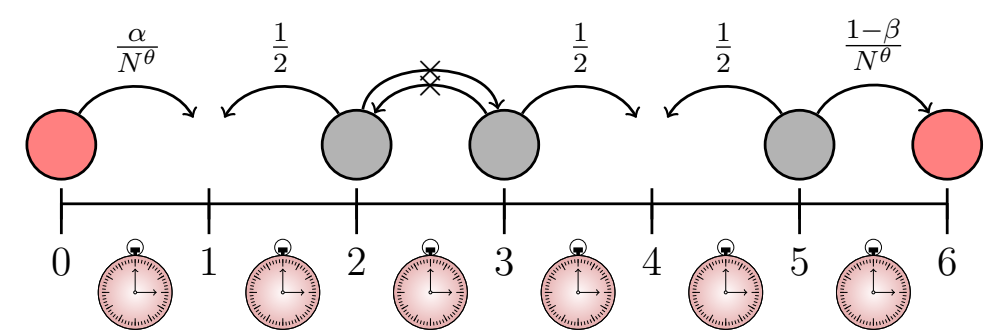

Figura 2.9: Configuração com seus possíveis saltos e taxas referentes ao SSEP em contato com reservatórios lentos.

\section{3}

\section{Construção de Harris}

Nesta seção faremos a construção gráfica ou construção de Harris para o PMM em contato com reservatórios. Tal construção, desenvolvida por Ted Harris em [12], é muito útil para construir processos de exclusão explicitamente em termos de famílias de processos de Poisson independentes.

Como vimos na seção anterior, $\Sigma_{N}=\{1, \cdots, N-1\}$ é o conjunto dos sítios onde distribuiremos as nossas partículas, e o produto cartesiano $\{0,1\}^{\Sigma_{N}}$ é o espaço de estados do nosso processo. Para cada elo $\{x, x+1\}$ com $x=1, \cdots, N-2$ associamos três relógios de Poisson, que serão representados pelos seguintes processos de Poisson: $N_{x, x+1}^{x-1}(t)$ com parâmetro $1, N_{x, x+1}^{x-2}(t)$ com parâmetro 1, e $N_{x, x+1}(t)$ com parâmetro 2. Associamos também processos de Poisson aos elos $\{0,1\}$ e $\{N-1, N\}$, onde em cada um desses elos teremos dois processos de Poisson associados: $N_{0,1}(t)$ com parâmetro $\alpha, N_{1,0}(t)$ com parâmetro $1-\alpha, N_{N-1, N}(t)$ com parâmetro $\beta$ e $N_{N, N-1}(t)$ com parâmetro $1-\beta$. Todos esses processos são independentes, e $\alpha, \beta \in(0,1)$. Faremos agora uma representação bi-dimensional no espaço-tempo $\Sigma_{N} \cup\{0, N\} \times[0, \infty)$, de modo que possamos representar a evolução temporal do sistema graficamente. Para cada $x \in \Sigma_{N} \cup\{0\}$ anexaremos ao ponto $\left(\frac{2 x+1}{2}, 0\right)$ um eixo vertical orientado para baixo, representando os processos de Poisson associados ao elo $\{x, x+1\}$. Assim, pensando no espaço-tempo como sendo $\Sigma_{N} \cup\{0, N\} \times[0, \infty)$, onde o eixo das abscisas está orientado para baixo, para cada tempo de salto $t$ determinado por cada um dos processos de Poisson desenhamos uma certa marca no ponto $\left(\frac{2 x+1}{2}, t\right) \in \Sigma_{N} \cup\{0, N\} \times[0, \infty)$. Essas marcas referentes a 
cada um dos processos são dadas por

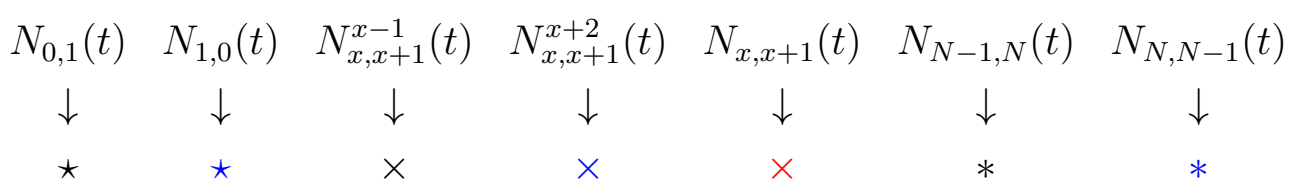

No tempo $t=0$, desenharemos a configuração inicial $\eta_{0} \in\{0,1\}^{\Sigma_{N}}$ em $\Sigma_{N}$, como na Figura 2.10. Desta forma, após o início do processo, cada partícula em um sítio $x \in \Sigma_{N}$ traça uma trajetória no espaço-tempo. A trajetória é descrita da seguinte maneira: se o sistema encontra uma marca vinda de um dos processos nas fronteiras, por exemplo, a marca $\star$, vinda do processo de Poisson $N_{0,1}(t)$, então uma partícula entra para o bulk através do sítio $1 \mathrm{com}$ uma taxa $\alpha$ se não tivermos nenhuma partícula no sítio 1, e as partículas movem-se para baixo com a taxa associada a este relógio. Se o toque do relógio vem do processo de Poisson $N_{1,0}(t)$ e existe uma partícula no sítio 1 , então a partícula sai do bulk através do sítio 0 com uma taxa $1-\alpha$ e movem-se para baixo com a taxa associada a este relógio. Para a outra fronteira a descrição é análoga. No bulk, se um dos relógios associados ao elo $\{x, x+1\}$ tocar e o elo não se encontrar entre a marca do referido relógio, as partículas da nossa configuração vão mover-se para baixo com a taxa associada a esse relógio, e quando o elo $\{x, x+1\}$ encontrar-se entre a marca do referido relógio no ponto $\left(\frac{2 x+1}{2}, t\right)$, as partículas do elo trocarão de posição e vão mover-se para baixo com a taxa associada a este relógio com as seguintes condições:

- Processo de Poisson $N_{x, x+1}^{x-1}(t)$. Se existir uma partícula no sítio $x-1$ e nenhuma partícula no sítio $x+2$.

- Processo de Poisson $N_{x, x+1}^{x+2}(t)$. Se existir uma partícula no sítio $x+2$ e nenhuma partícula no sítio $x-1$.

- Processo de Poisson $N_{x, x+1}(t)$. Se existir uma partícula no sítio $x-1$ e uma partícula no sítio $x+2$.

Utilizando as ideias apresentadas acima, faremos a seguir a construção gráfica para o SSEP e o PMM, ambos em contato com reservatórios, com espaço de estados $\{0,1\}^{\Sigma_{4}}$ e com configuração inicial $\eta_{0} \in\{0,1\}^{\Sigma_{4}}$ dada por

$\eta_{0}$

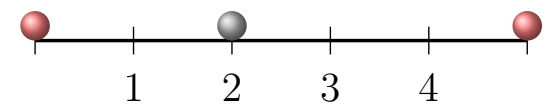

Figura 2.10: Representação da configuração inicial $\eta_{0}$.

Vimos acima a descrição de como construir graficamente o PMM em contato com reservatórios. Para o SSEP em contato com reservatórios teremos 
os mesmos relógios que o PMM associados aos elos $\{0,1\}$ e $\{N-1, N\}$, e para cada um dos elos no bulk associaremos um processo de Poisson de parâmetro 1, cujo toque do relógio associado a este processo será representado pela marca $\times$. O salto das partículas no bulk acontecerão da seguinte maneira: se o relógio associado a um elo $\{x, x+1\}$ toca, as partículas no elo trocam imediatamente de posição com taxa 1 e movem-se para baixo com essa taxa.

Os tempos de salto de cada um dos processos de Poisson associados aos elos são apresentados na tabela abaixo, onde cada coluna representa os tempos de toque dos relógios em cada um dos elos do nosso sistema, e cada um desses processos de Poisson foi gerado utilizando o algoritmo apresentado no Apêndice C.

\begin{tabular}{ccccc}
\hline$\{0,1\}$ & $\{1,2\}$ & $\{2,3\}$ & $\{3,4\}$ & $\{4,5\}$ \\
\hline 1.15 & 0.58 & 0.02 & 1.25 & 0.25 \\
1.23 & 1.54 & 0.82 & 3.05 & 0.32 \\
2.41 & 1.64 & 1.26 & 3.37 & 1.85 \\
4.05 & 4.30 & 4.43 & 3.85 & 4.15 \\
\hline
\end{tabular}

Tabela 2.1: Tempo em que cada relógio de Poisson toca.
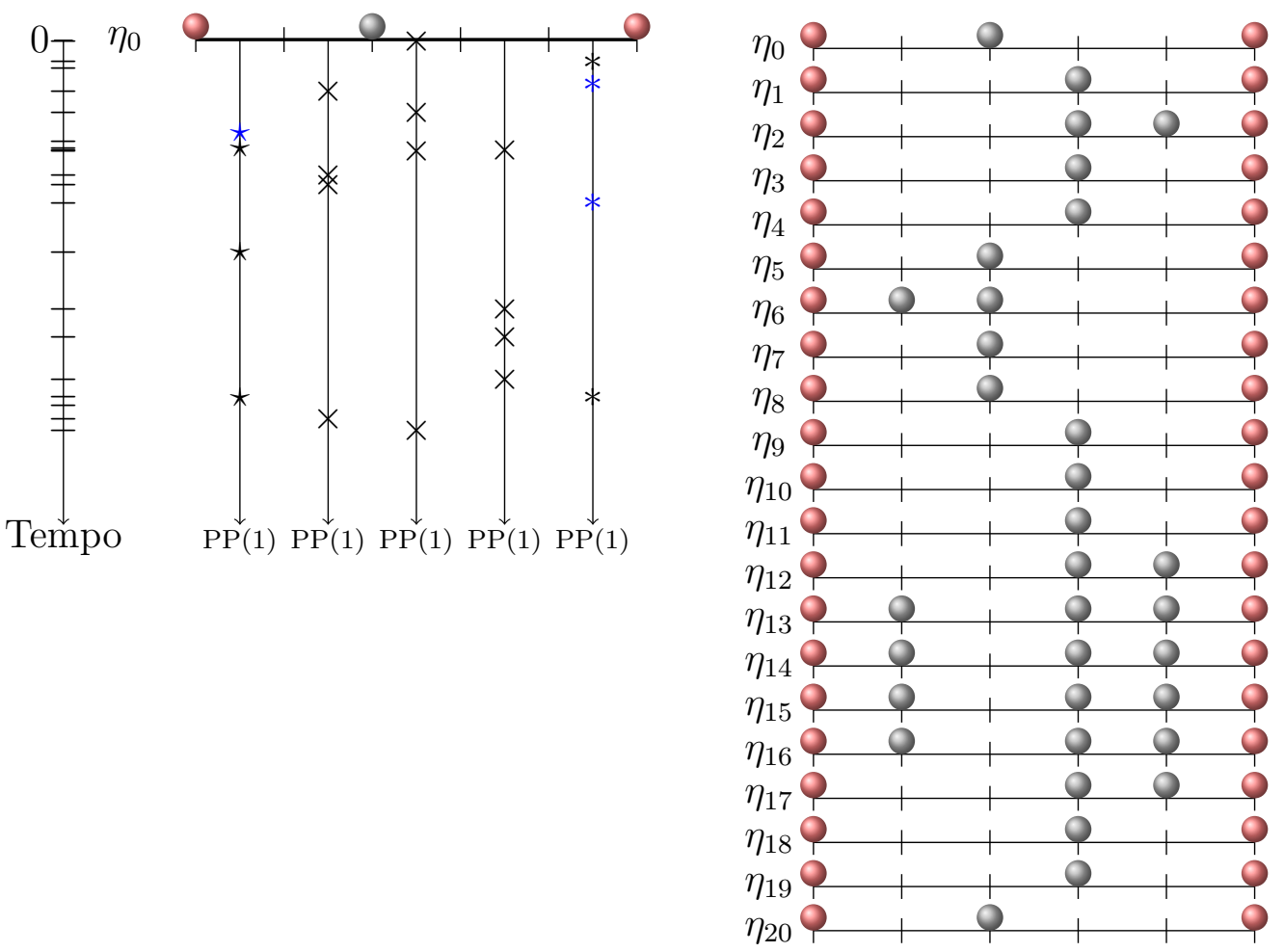

Figura 2.11: SSEP em contato com reservatórios. 

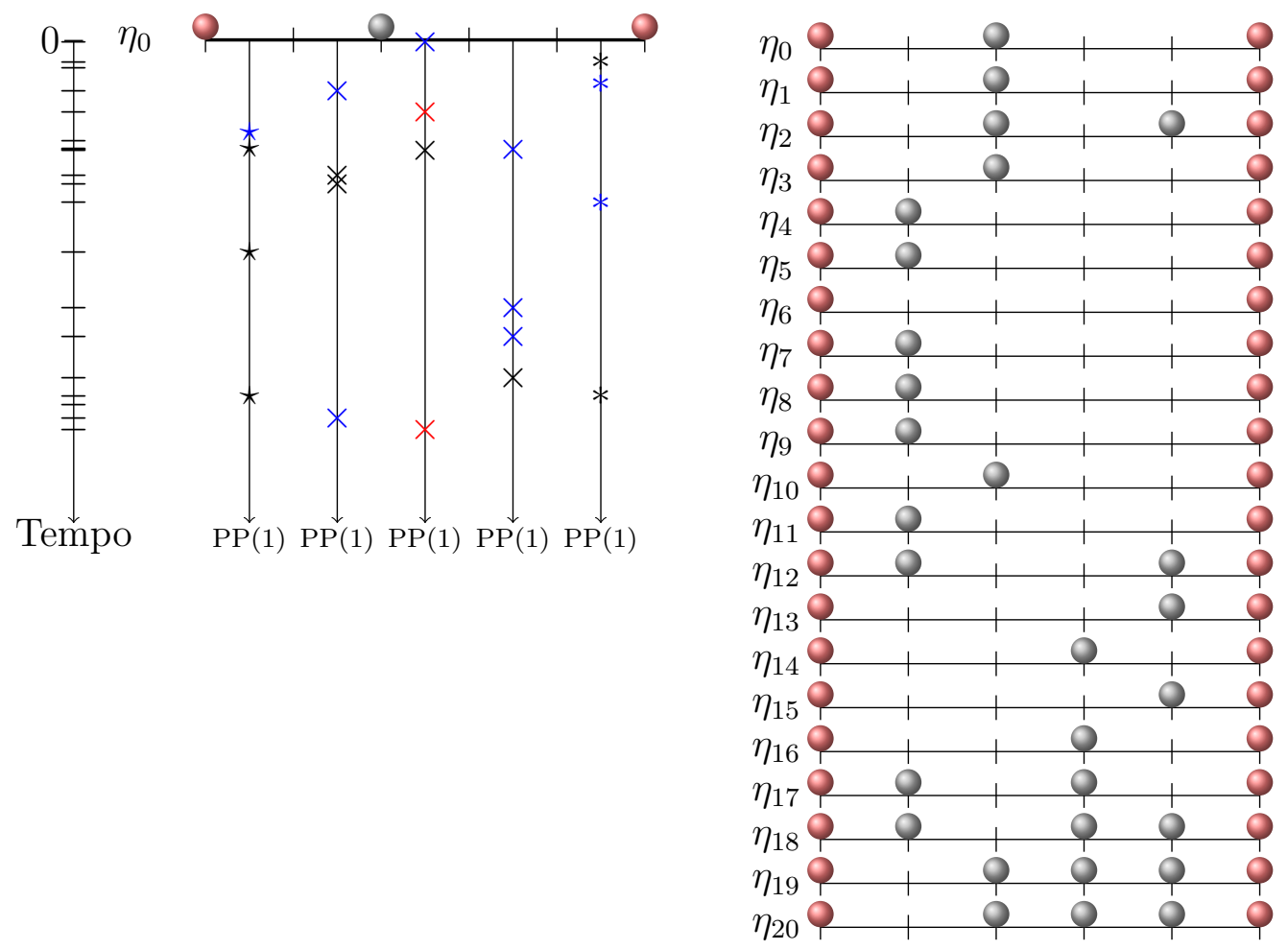

Figura 2.12: PMM em contato com reservatórios.

Agora vamos analisar as figuras acima. A configuração inicial para cada um dos processos é dada por $\eta_{0}$, e o primeiro toque do relógio está associado ao elo $\{2,3\}$. Por um lado, como a configuração inicial $\eta_{0}$ tem uma partícula no sítio 2 e nenhuma partícula no sítio 3 , as partículas trocam de posição no elo, como pode ser observado na Figura 2.11, e chamamos esta nova configuração do SSEP em contato com reservatórios de $\eta_{1}$. Por outro lado, como em $\eta_{0}$ não temos partículas no sítio 1 nem no sítio 4, o salto não acontece no PMM em contato com reservatórios, como vemos na Figura 2.12, e dessa forma a nova configuração $\eta_{1}$ coincide com $\eta_{0}$. O segundo toque do relógio está associado ao elo $\{4,5\}$. Uma vez que o sítio 4 de ambas as configurações $\eta_{1}$ dos processos está vazio, como podemos ver na Figura 2.11 e Figura 2.12, uma partícula entra para este sítio e assim obtemos uma nova configuração $\eta_{2}$ para cada um dos processos.

Vale ressaltar neste ponto uma diferença simples entre os dois processos. No SSEP em contato com reservatórios, sempre que um relógio associado ao elo $\{x, x+1\}$ tocar, e simultaneamente uma única partícula estiver em um dos sítios do elo, o salto ocorre. Já no PMM em contato com reservatórios isso não acontece, uma vez que o salto das partículas no elo $\{x, x+1\}$ está condicionado à existência de partículas nos sítios $x-1$ e $x+2$. Essa diferença pode ser observada na transição da configuração $\eta_{0}$ para a configuração $\eta_{1}$ 
apresentada nas figuras acima.

\section{4}

\section{Construção via gerador infinitesimal}

A descrição mais natural de um processo de Markov com espaço de estados finito é dada pelas probabilidades de transição $P_{t}(\eta, \xi)$, que representam a probabilidade do processo estar no estado $\xi$ no tempo $t$, dado que estava no estado $\eta$ no tempo 0 . Tendo em vista a grande dificuldade para o cálculo explícito dessas probabilidades de transição, uma boa descrição para este processo de Markov é dada pelo gerador infinitesimal, que é um operador linear que codifica toda a informação do processo. Para mais detalhes o leitor pode consultar [17], Capítulo 3, e o Apêndice A.

A dinâmica retratada anteriormente na Seção 2.2 será descrita agora por meio do gerador infinitesimal associado ao processo de Markov exposto acima. Para tal, relembre que $N \geq 1$ e $\Sigma_{N}=\{1, \cdots, N-1\}$. Os elementos de $\Sigma_{N}$ são chamados sítios. O espaço $\Omega_{N}:=\{0,1\}^{\Sigma_{N}}$ chamado espaço de configurações, é o espaço microscópico, cujos elementos são vetores com $N-1$ coordenadas, compostos somente de zeros e uns e chamados de configurações. Este espaço consiste no espaço de estados do processo de Markov. Assim, uma configuração $\eta \in\{0,1\}^{\Sigma_{N}}$ é uma função $\eta: \Sigma_{N} \rightarrow\{0,1\}$, onde $\eta(x) \in\{0,1\}$ representa o número de partículas no sítio $x$ para a configuração $\eta$, isto é, $\eta(x)=0$ quer dizer que o sítio $x$ da configuração $\eta$ está vazio e $\eta(x)=1$ diz que o sítio $x$ está ocupado.

Fixamos $\alpha, \beta \in(0,1)$. O PMM em contato com reservatórios é um processo de Markov $\left\{\eta_{t}\right\}_{t \geq 0}$ com espaço de estados $\Omega_{N}:=\{0,1\}^{\Sigma_{N}}$. Este processo pode ser caracterizado em termos do seu gerador infinitesimal $L_{N}$, o qual definimos como segue. Dada uma função $f: \Omega_{N} \rightarrow \mathbb{R}$, e com a convenção

$$
\eta(0)=\alpha \text { e } \eta(N)=\beta,
$$

seja

$$
\left(L_{N} f\right)(\eta)=\left(L_{N, b u l k} f\right)(\eta)+\left(L_{N, b} f\right)(\eta)
$$

onde,

$$
\begin{gathered}
\left(L_{N, b u l k} f\right)(\eta)=\sum_{x=1}^{N-2} c_{x}\left(\eta, \eta^{x, x+1}\right)\left[f\left(\eta^{x, x+1}\right)-f(\eta)\right], \\
\left(L_{N, b} f\right)(\eta)=l_{\alpha}(\eta)\left[f\left(\eta^{1}\right)-f(\eta)\right]+l_{\beta}(\eta)\left[f\left(\eta^{N-1}\right)-f(\eta)\right], \\
l_{\alpha}(\eta)=\alpha(1-\eta(1))+(1-\alpha) \eta(1), \\
l_{\beta}(\eta)=\beta(1-\eta(N-1))+(1-\beta) \eta(N-1),
\end{gathered}
$$




$$
c_{x}\left(\eta, \eta^{x, x+1}\right)=[\eta(x)(1-\eta(x+1))+\eta(x+1)(1-\eta(x))][\eta(x-1)+\eta(x+2)],
$$

$\eta^{x, x+1}$ é a configuração obtida de $\eta$ trocando os seus valores nos sítios $x$ e $x+1$ :

$$
\left(\eta^{x, x+1}\right)(y)=\left\{\begin{array}{cl}
\eta(x+1), & \text { se } y=x \\
\eta(x), & \text { se } y=x+1 \\
\eta(y), & \text { caso contrário }
\end{array}\right.
$$

e para $x=1$ ou $x=N-1, \eta^{x}$ é a configuração obtida de $\eta$ trocando o seu valor no sítio $x$, isto é

$$
\left(\eta^{x}\right)(y)=\left\{\begin{array}{cl}
1-\eta(x), & \text { se } y=x \\
\eta(y), & \text { caso contrário. }
\end{array}\right.
$$

Para ilustrar a ação do gerador infinitesimal definido acima, consideremos $N=6$ e $\eta \in \Sigma_{6}$ uma configuração representada na figura abaixo, onde explicitamos os possíveis saltos e as taxas referentes a cada um deles.

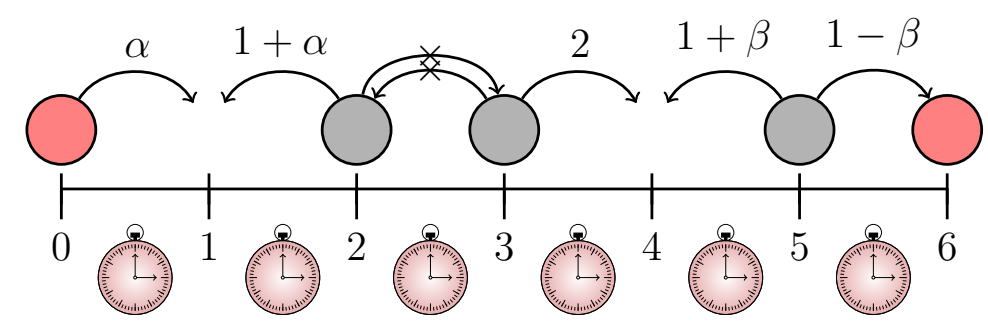

Figura 2.13: Configuração com seus possíveis saltos e taxas referentes ao PMM em contato com reservatórios.

Fazendo uma caracterização de $\eta$ utilizando o gerador infinitesimal definido em (2.3), temos

$$
\begin{aligned}
\left(L_{N, b u l k} f\right)(\eta) & =\sum_{x=1}^{4} c_{x}\left(\eta, \eta^{x, x+1}\right)\left[f\left(\eta^{x, x+1}\right)-f(\eta)\right] \\
& =(\alpha+1)\left[f\left(\eta^{1,2}\right)-f(\eta)\right]+2\left[f\left(\eta^{3,4}\right)-f(\eta)\right] \\
& +(1+\beta)\left[f\left(\eta^{4,5}\right)-f(\eta)\right] \\
& =(\alpha+1) f\left(\eta^{1,2}\right)+2 f\left(\eta^{3,4}\right)+(1+\beta) f\left(\eta^{4,5}\right)-(4+\alpha+\beta) f(\eta), \\
\left(L_{N, b} f\right)(\eta) & =[\alpha(1-\eta(1))+(1-\alpha) \eta(1)]\left[f\left(\eta^{1}\right)-f(\eta)\right] \\
& +[\beta(1-\eta(5))+(1-\beta) \eta(5)]\left[f\left(\eta^{5}\right)-f(\eta)\right] \\
& =\alpha\left[f\left(\eta^{1}\right)-f(\eta)\right]+(1-\beta)\left[f\left(\eta^{5}\right)-f(\eta)\right] \\
& =\alpha f\left(\eta^{1}\right)+(1-\beta) f\left(\eta^{5}\right)-(1+\alpha-\beta) f(\eta) .
\end{aligned}
$$

Com os resultados apresentados nessa seção, será que somos capazes de 
representar a ação do gerador infinitesimal matricialmente? A próxima Seção mostrará que sim, uma vez que $N$ é finito.

\section{5}

\section{Representação matricial}

Dado o gerador infinitesimal $L_{N}$ definido em (2.3), podemos expressar sua ação através de uma matriz $Q_{N}:=L_{N, \text { bulk }}+L_{N, b}$, chamada matriz de taxas, que consiste em uma matriz quadrada $2^{N-1} \times 2^{N-1}$ com as seguintes entradas

$$
Q_{N}(\eta, \xi)=\left[L_{N, b u l k}\right](\eta, \xi)+\left[L_{N, b}\right](\eta, \xi)
$$

onde, utilizando a convenção (2.1), temos

$$
\left[L_{N, \text { bulk }}\right](\eta, \xi)= \begin{cases}\eta(x-1)+\eta(x+2), & \text { se } \xi=\eta^{x, x+1}, 1 \leq x \leq N-2, \xi \neq \eta \\ 0, & \text { se } \xi \neq \eta^{x, x+1}, 1 \leq x \leq N-2, \\ -\sum_{\zeta: \zeta \neq \eta}\left(L_{N, b u l k}\right)(\eta, \zeta), & \text { se } \xi=\eta\end{cases}
$$

$\mathrm{e}$

$$
\left[L_{N, b}\right](\eta, \xi)= \begin{cases}l_{\alpha}(\eta), & \text { se } \xi=\eta^{1} \\ l_{\beta}(\eta), & \text { se } \xi=\eta^{N-1} \\ -\left(l_{\alpha}+l_{\beta}\right)(\eta), & \text { se } \xi=\eta \\ 0, & \text { caso contrário }\end{cases}
$$

onde $l_{\alpha}(\eta)$ e $l_{\beta}(\eta)$ são dadas em (2.4) e (2.5) respectivamente. Assim, considerando $f=(f(\eta))_{\eta \in\{0,1\}^{\Sigma_{N}}}$ como um vetor coluna, temos que Notamos que esta matriz não é uma matriz de transição, visto que há entradas negativas e a soma de cada linha é zero. Para maiores detalhes sobre essas matrizes vide [18], página 58. Ilustraremos a representação matricial acima com o próximo exemplo.

Exemplo 2.2. Sejam $\Sigma_{4}=\{1,2,3\}$ e $f: \Omega_{4} \rightarrow \mathbb{R}$. De (2.3) o gerador infinitesimal para uma configuração $\eta \in\{0,1\}^{\Sigma_{4}}$ é dado por

$$
\begin{aligned}
\left(L_{4, b u l k} f\right)(\eta) & =[\eta(1)(1-\eta(2))+\eta(2)(1-\eta(1))][\alpha+\eta(3)]\left[f\left(\eta^{1,2}\right)-f(\eta)\right] \\
& +[\eta(2)(1-\eta(3))+\eta(3)(1-\eta(2))][\eta(1)+\beta]\left[f\left(\eta^{2,3}\right)-f(\eta)\right], \\
\left(L_{4, b} f\right)(\eta) & =[\alpha(1-\eta(1))+(1-\alpha) \eta(1)]\left[f\left(\eta^{1}\right)-f(\eta)\right] \\
& +[\beta(1-\eta(3))+(1-\beta) \eta(3)]\left[f\left(\eta^{3}\right)-f(\eta)\right] .
\end{aligned}
$$


Todas as possíveis configurações $\eta_{1}, \cdots, \eta_{8}$ com as indicações das taxas de transição podem ser vistas abaixo
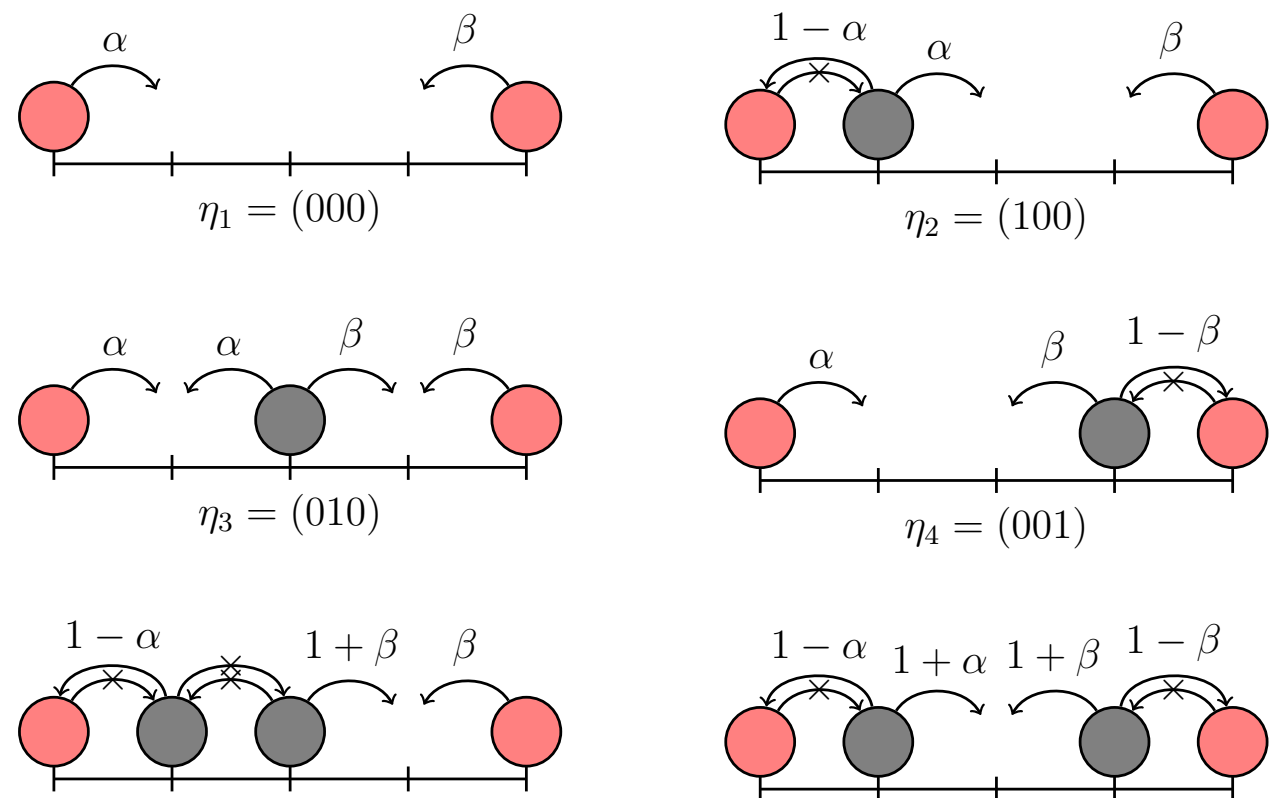

$\eta_{5}=(110)$
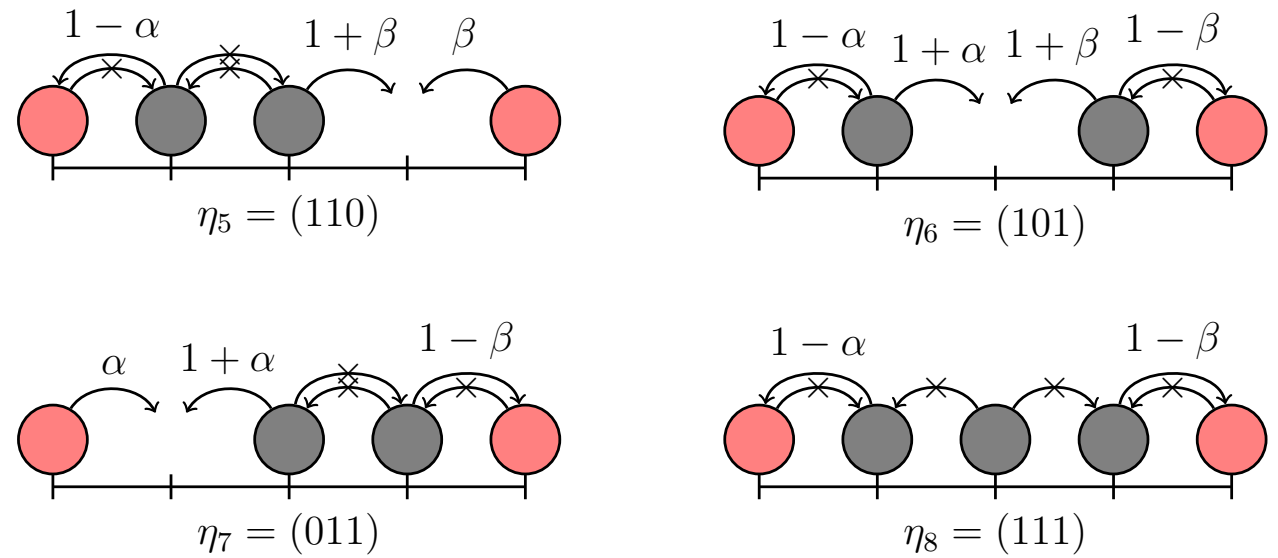

$\eta_{8}=(111)$

Observação 2.3. Os índices das configurações presentes na figura acima não dizem respeito ao tempo, fazemos este abuso de notação aqui para distinguirmos uma configuração da outra e tornar a apresentação mais simples. Nos capítulos seguintes, sempre que usarmos a notação $\eta_{t}$, estaremos fazendo referência ao valor da configuração $\eta$ no tempo t.

Com as informações acima, vamos construir nossa matriz de taxas. Começamos estudando como obter os valores da primeira e segunda linha da matriz $\left[L_{4, b u l k}\right]$, e depois repetiremos o processo para as linhas remanescentes. O cálculo de $\left[L_{4, b}\right]$ será feito em seguida.

De fato, $\left[L_{4, \text { bulk }}\right]\left(\eta_{1}, \eta_{2}\right)=\cdots=\left[L_{4, \text { bulk }}\right]\left(\eta_{1}, \eta_{8}\right)=0$, pois $\eta_{m} \neq \eta_{1} \mathrm{e}$ $\eta_{m} \neq \eta_{1}^{x, x+1}$ para $1 \leq x \leq 2$ e $2 \leq m \leq 8$, isto é, $\eta_{1}$ é uma configuração bloqueada, como visto na Seção 2.2. Vemos também que $\left[L_{4, b u l k}\right]\left(\eta_{1}, \eta_{1}\right)=0$, pois é a soma das entradas obtidas anteriormente com o sinal trocado. Portanto a primeira linha da nossa matriz é constituída por zeros. Fazendo uma análise 
na segunda linha da matriz observamos que $\eta_{3}=\eta_{2}^{1,2}$ e $\eta_{3} \neq \eta_{2}$, então $\left[L_{4, \text { bulk }}\right]\left(\eta_{2}, \eta_{3}\right)=1+\alpha$. Como a partir de qualquer configuração diferente de $\eta_{2}$ não conseguimos obter a configuração $\eta_{2}$ trocando somente partículas no bulk, segue que $\left[L_{4, \text { bulk }}\right]\left(\eta_{2}, \eta_{m}\right)=0$ para $m \in\{1,4,5,6,7,8\}$ e $\left[L_{4, \text { bulk }}\right]\left(\eta_{2}, \eta_{2}\right)=$ $-1-\alpha$. Logo, utilizando um raciocínio análogo ao anterior, resulta

$$
\left[\begin{array}{ccccccccc}
\eta_{1} & \eta_{2} & \eta_{3} & \eta_{4} & \eta_{5} & \eta_{6} & \eta_{7} & \eta_{8} \\
\eta_{1} & \eta_{2} & 0 & 0 & 0 & 0 & 0 & 0 & 0 \\
\eta_{3} & 0 & -1-\alpha & 1+\alpha & 0 & 0 & 0 & 0 & 0 \\
& 0 & 1+\alpha & -2-\alpha-\beta & 1+\beta & 0 & 0 & 0 & 0 \\
\eta_{4} & 0 & 0 & 1+\beta & -1-\beta & 0 & 0 & 0 & 0 \\
\eta_{5} & 0 & 0 & 0 & 0 & -1-\beta & 1+\beta & 0 & 0 \\
\eta_{6} & 0 & 0 & 0 & 0 & 1+\beta & -2-\alpha-\beta & 1+\alpha & 0 \\
\eta_{7} & 0 & 0 & 0 & 0 & 0 & 1+\alpha & -1-\alpha & 0 \\
\eta_{8} & 0 & 0 & 0 & 0 & 0 & 0 & 0
\end{array}\right) .
$$

Faremos agora uma análise para a primeira linha de $\left[L_{4, b}\right]$. Primeiro observemos que $l_{\alpha}\left(\eta_{1}\right)=\alpha$ e $l_{\beta}\left(\eta_{1}\right)=\beta$. De fato, $\left[L_{4, b}\right]\left(\eta_{1}, \eta_{m}\right)=0$, pois $\eta_{m} \neq \eta_{1}$ para $m \in\{3,5,6,7,8\}$. Como $\eta_{1}=\eta_{2}^{1}$ e $\eta_{1}=\eta_{4}^{1}$, segue que $\left[L_{4, b}\right]\left(\eta_{1}, \eta_{2}\right)=\alpha$ e $\left[L_{4, b}\right]\left(\eta_{1}, \eta_{4}\right)=\beta$ respectivamente. Por fim, $\left[L_{4, b}\right]\left(\eta_{1}, \eta_{1}\right)=-(\alpha+\beta)$. Utilizando um raciocínio análogo ao anterior temos a seguinte matriz

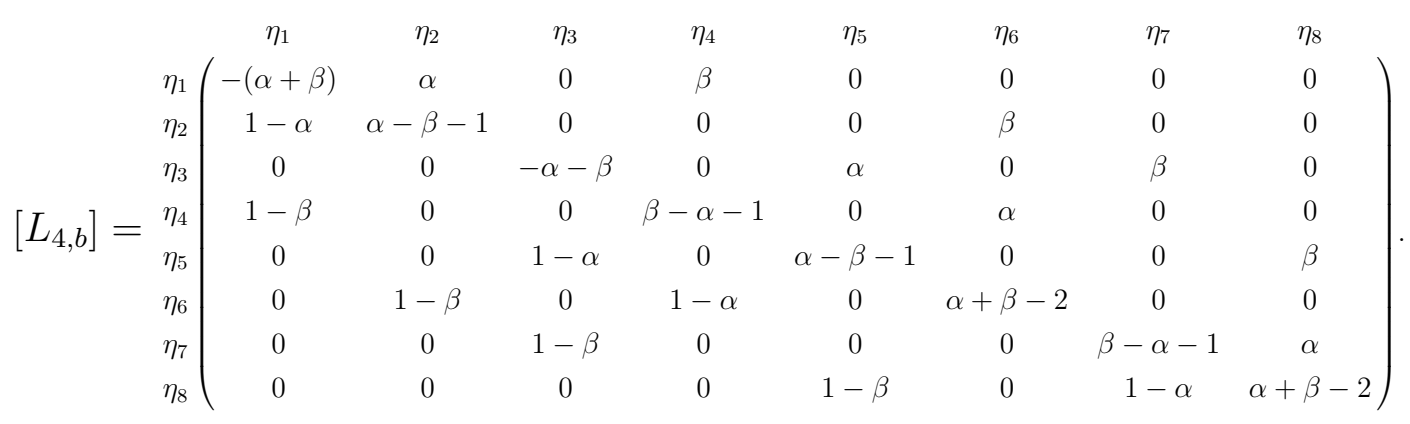

A matriz $Q_{4}$ é obtida fazendo a soma das duas matrizes acima, e portanto, é dada por 


\begin{tabular}{|c|c|c|c|c|c|c|c|}
\hline$\eta_{1}$ & $\eta_{2}$ & $\eta_{3}$ & $\eta_{4}$ & $\eta_{5}$ & $\eta_{6}$ & $\eta_{7}$ & $\eta_{8}$ \\
\hline$\eta_{1}(-(\alpha+\beta)$ & $\alpha$ & 0 & $\beta$ & 0 & 0 & 0 & 0 \\
\hline $1-\alpha$ & $-2-\beta$ & $1+\alpha$ & 0 & 0 & $\beta$ & 0 & 0 \\
\hline$\eta_{3}$ & $1+\alpha$ & $-2-2 \alpha-2 \beta$ & $1+\beta$ & $\alpha$ & 0 & $\beta$ & 0 \\
\hline $1-\beta$ & 0 & $1+\beta$ & $-2-\alpha$ & 0 & $\alpha$ & 0 & 0 \\
\hline 0 & 0 & $1-\alpha$ & 0 & $-2-2 \beta+\alpha$ & $1+\beta$ & 0 & $\beta$ \\
\hline$\eta_{6}$ & $1-\beta$ & 0 & $1-\alpha$ & $1+\beta$ & -4 & $1+\alpha$ & 0 \\
\hline 0 & 0 & $1-\beta$ & 0 & 0 & $1+\alpha$ & $-2 \beta-2 \alpha$ & $\alpha$ \\
\hline$\eta_{8}$ & 0 & 0 & 0 & $1-\beta$ & 0 & $1-\alpha$ & $\alpha+\beta-2$ \\
\hline
\end{tabular}

\section{6}

\section{Medidas estacionárias}

Começaremos agora nossa procura pelas medidas estacionárias do PMM em contato com reservatórios. Mostraremos que quando $\alpha=\beta$ a medida estacionária do modelo é a medida Bernoulli produto.

Definição 2.4. Um processo de Markov $\left(X_{t}\right)_{t \geq 0}$ com probabilidades de transição $\left(P_{t}\right)_{t \geq 0}$ é dito reversível com respeito à medida $\mu$ no seu espaço de estados se a equação de balanço $\mu(\eta) P_{t}(\eta, \xi)=\mu(\xi) P_{t}(\xi, \eta)$ for satisfeita para quaisquer estados $\eta, \xi$ e $t>0$.

Segue da definição acima que qualquer medida de probabilidade $\mu$ satisfazendo a equação de balanço é uma medida estacionária. Mais detalhes podem ser vistos, por exemplo, em [18], página 79.

Definição 2.5. Seja $0<\alpha<1$. Chamamos de medida Bernoulli produto de parâmetro $\alpha$ à medida $\nu_{\alpha}^{N}$ em $\{0,1\}^{\Sigma_{N}}$ tal que fixado $\eta \in\{0,1\}^{\Sigma_{N}}$, as variáveis aleatórias $\{\eta(x)\}_{x \in \Sigma_{N}}$ são independentes e

$$
\nu_{\alpha}^{N}\left\{\eta \in\{0,1\}^{\Sigma^{N}}: \eta(x)=1\right\}=\alpha .
$$

Assim, para uma configuração $\eta$ se tem

$$
\nu_{\alpha}^{N}(\eta)=\alpha^{\sum_{x=1}^{N-1} \eta(x)}(1-\alpha)^{\sum_{x=1}^{N-1}(1-\eta(x))} .
$$

Proposição 2.6. Se $\alpha=\beta$ então $\nu_{\alpha}^{N}$ é estacionária e reversível.

Demonstração. Basta verificarmos que $\nu_{\alpha}^{N}(\eta) Q_{N}(\eta, \xi)=\nu_{\alpha}^{N}(\xi) Q_{N}(\xi, \eta)$ para quaisquer configurações $\eta, \xi \in\{0,1\}^{\Sigma_{N}}$.

- No caso em que $\xi=\eta$ a igualdade é clara.

- Se $\xi \notin\left\{\eta, \eta^{1}, \eta^{N-1}, \eta^{x, x+1}\right\}$ para $x \in \Sigma_{N}$, então $Q_{N}(\eta, \xi)=Q_{N}(\xi, \eta)=0$, o que verifica a condição. 
- Se $\xi=\eta^{x, x+1}$ para algum $x \in \Sigma_{N}$, temos $Q_{N}(\eta, \xi)=Q_{N}(\xi, \eta)=$ $\eta(x-1)+\eta(x+2)$ e $\nu_{\alpha}^{N}(\eta)=\nu_{\alpha}^{N}\left(\eta^{x, x+1}\right)$, uma vez que $\nu_{\alpha}^{N}$ não depende das posições das partículas e sim da quantidade de partículas.

- Quando $\xi=\eta^{1}$ e $\eta(1)=0$ temos $Q_{N}(\eta, \xi)=\alpha, Q_{N}(\xi, \eta)=1-\alpha$ e $\nu_{\alpha}^{N}(\xi)=\frac{\alpha}{1-\alpha} \nu_{\alpha}^{N}(\eta)$, os quais cumprem a equação de balanço. Se $\eta(1)=1$ temos $Q_{N}(\eta, \xi)=1-\alpha, Q_{N}(\xi, \eta)=\alpha$ e $\nu_{\alpha}^{N}(\xi)=\frac{1-\alpha}{\alpha} \nu_{\alpha}^{N}(\eta)$, que satisfaz mais uma vez a equação de balanço.

- O caso $\xi=\eta^{N-1}$ pode ser verificado procedendo-se de maneira análoga à anterior.

Quando $\alpha \neq \beta$, como temos um processo de Markov definido em um espaço de estados finito, por [15] sabemos que existe uma medida estacionária que denotaremos por $\mu_{s s}$ que já não é uma medida produto como no caso em que $\alpha=\beta$. Dessa forma, no Capítulo 5 , utilizando o método da matriz ansatz, conseguiremos obter informações sobre essa medida. 


\section{Heurística da equação hidrodinâmica}

O objetivo deste capítulo consiste em obtermos heuristicamente a equação hidrodinâmica para o PMM em contato com reservatórios. Neste sentido, na Seção 3.1, calcularemos a corrente microscópica para o PMM em contato com reservatórios. Depois, na Seção 3.2, apresentaremos a dedução heurística da equação hidrodinâmica para este modelo. Finalmente, na Seção 3.3, encontraremos a formulação da solução fraca para a equação em meios porosos com condições de Dirichlet e mostraremos que essa solução é única.

\section{1}

\section{Corrente microscópica}

Exemplo 3.1. Seja $z \in \Sigma_{N}$ e $f_{z}:\{0,1\}^{\Sigma_{N}} \rightarrow\{0,1\}$ definida por $f_{z}(\eta)=\eta(z)$.

Calculemos $\left(L_{N} f_{z}\right)(\eta)$.

Aqui vamos utilizar a convenção feita em (2.1), e para simplificar os cálculos também utilizaremos que,

$$
\eta(x)(1-\eta(x+1))+\eta(x+1)(1-\eta(x))=(\eta(x)-\eta(x+1))^{2} .
$$

Assim, para $z \in\{2, \cdots, N-2\}$ temos

$$
\begin{aligned}
L_{N, b u l k} \eta(z) & =\sum_{x=1}^{N-2}(\eta(x)-\eta(x+1))^{2}(\eta(x-1)+\eta(x+2))\left[\eta^{x, x+1}(z)-\eta(z)\right] \\
& =(\eta(z-1)-\eta(z))^{2}(\eta(z-2)+\eta(z+1))\left[\eta^{z-1, z}(z)-\eta(z)\right] \\
& +(\eta(z)-\eta(z+1))^{2}(\eta(z-1)+\eta(z+2))\left[\eta^{z, z+1}(z)-\eta(z)\right] \\
& =(\eta(z-1)-\eta(z))^{2}(\eta(z-2)+\eta(z+1))[\eta(z-1)-\eta(z)] \\
& +(\eta(z)-\eta(z+1))^{2}(\eta(z-1)+\eta(z+2))[\eta(z+1)-\eta(z)] \\
& =(\eta(z-1)-\eta(z))^{3}(\eta(z-2)+\eta(z+1)) \\
& -(\eta(z)-\eta(z+1))^{3}(\eta(z-1)+\eta(z+2)) \\
& =(\eta(z-1)-\eta(z))(\eta(z-2)+\eta(z+1)) \\
& -(\eta(z)-\eta(z+1))(\eta(z-1)+\eta(z+2)),
\end{aligned}
$$




$$
\begin{aligned}
L_{N, b} \eta(z) & =[\alpha(1-\eta(1))+(1-\alpha) \eta(1)]\left[\eta^{1}(z)-\eta(z)\right] \\
& +[\beta(1-\eta(N-1))+(1-\beta) \eta(N-1)]\left[\eta^{N-1}(z)-\eta(z)\right] \\
& =0 .
\end{aligned}
$$

A última expressão é igual a zero pois $z \neq 1$ e $z \neq N-1$, consequentemente $\eta^{1}(z)=\eta^{N-1}(z)=\eta(z)$.

Caso $z=1$ :

$$
\begin{aligned}
L_{N, \text { bulk }} \eta(1) & =(\eta(1)-\eta(2))^{2}(\alpha+\eta(3))\left[\eta^{1,2}(1)-\eta(1)\right] \\
& =(\eta(1)-\eta(2))^{2}(\alpha+\eta(3))[\eta(2)-\eta(1)] \\
& =(\eta(2)-\eta(1))^{3}(\alpha+\eta(3)) \\
& =(\eta(2)-\eta(1))(\alpha+\eta(3)), \\
L_{N, b} \eta(1) & =[\alpha(1-\eta(1))+(1-\alpha) \eta(1)]\left[\eta^{1}(1)-\eta(1)\right] \\
& +[\beta(1-\eta(N-1))+(1-\beta) \eta(N-1)]\left[\eta^{N-1}(1)-\eta(1)\right] \\
& =[\alpha-\alpha \eta(1)+\eta(1)-\alpha \eta(1)][1-\eta(1)-\eta(1)]+0 \\
& =[\alpha+\eta(1)-2 \alpha \eta(1)][1-2 \eta(1)] \\
& =\alpha+\eta(1)-2 \alpha \eta(1)-2 \alpha \eta(1)-2 \eta(1)^{2}+4 \alpha \eta(1) \\
& =\alpha+\eta(1)-2 \eta(1)^{2} \\
& =\alpha-\eta(1) .
\end{aligned}
$$

Caso $z=N-1$ :

$$
\begin{aligned}
L_{N, b u l k} \eta(N-1) & =\sum_{x=1}^{N-2}(\eta(x)-\eta(x+1))^{2}(\eta(x-1)+\eta(x+2))\left[\eta^{x, x+1}(N-1)\right. \\
& -\eta(N-1)] \\
& =(\eta(N-2)-\eta(N-1))^{2}(\eta(N-3) \\
& +\beta)\left[\eta^{N-2, N-1}(N-1)-\eta(N-1)\right] \\
& =(\eta(N-2)-\eta(N-1))^{2}(\eta(N-3)+\beta)[\eta(N-2)-\eta(N-1)] \\
& =(\eta(N-2)-\eta(N-1))^{3}(\eta(N-3)+\beta) \\
& =(\eta(N-2)-\eta(N-1))(\eta(N-3)+\beta),
\end{aligned}
$$

$$
L_{N, b} \eta(N-1)=\beta-\eta(N-1)
$$

Portanto,

$$
L_{N} \eta(1)=\underbrace{(\alpha-\eta(1))}_{=j_{0,1}(\eta)}-\underbrace{(\eta(1)-\eta(2))(\alpha+\eta(3))}_{=j_{1,2}(\eta)},
$$




$$
\begin{aligned}
L_{N} \eta(z)= & \underbrace{(\eta(z-1)-\eta(z))(\eta(z-2)+\eta(z+1))}_{=j_{z-1, z}(\eta)} \\
- & \underbrace{(\eta(z)-\eta(z+1))(\eta(z-1)+\eta(z+2))}_{=j_{z, z+1}(\eta)} \\
& \operatorname{para} z \in\{2, \cdots, N-2\}, \\
L_{N} \eta(N-1)= & \underbrace{(\eta(N-2)-\eta(N-1))(\eta(N-3)+\beta)}_{=j_{N-2, N-1}(\eta)}-\underbrace{(\eta(N-1)-\beta)}_{=j_{N-1, N}(\eta)},
\end{aligned}
$$

onde $j_{z, z+1}(\eta)$ é a diferença entre a taxa de salto de $z$ para $z+1$ e a taxa de salto de $z+1$ para $z$, que representa a corrente microscópica associada ao elo $\{z, z+1\}$. Assim, para $z \in \Sigma_{N}, L_{N} \eta(z)$ pode ser escrito como

$$
L_{N} \eta(z)=j_{z-1, z}(\eta)-j_{z, z+1}(\eta)
$$

Aqui devemos fazer uma observação em relação à corrente calculada no bulk. A equação (3.1) é válida sempre que tivermos um sistema onde o número de partículas é conservado. Como no PMM as partículas no bulk só saltam para os possíveis vizinhos com uma certa taxa, então não se criam e nem se destroem partículas no bulk. Assim, dizemos que o número de partículas é conservado, e conseguimos escrever $L_{N} \eta(z)$ como (3.1).

Olhando mais uma vez para (3.1), observamos que a equação envolve o gradiente discreto da corrente microscópica. Desta forma, nossa ideia agora será escrever a corrente microscópica como o gradiente discreto de uma função $h: \Omega_{N} \rightarrow \mathbb{R}$, e para isso serão necessárias algumas manipulações. De fato, para $z \in\{1, \cdots, N-2\}$

$$
\begin{aligned}
j_{z, z+1}(\eta) & =(\eta(z)-\eta(z+1))(\eta(z-1)+\eta(z+2)) \\
& =\eta(z) \eta(z-1)+\eta(z) \eta(z+2)-\eta(z+1) \eta(z-1)-\eta(z+1) \eta(z+2)
\end{aligned}
$$

representa a corrente microscópica no bulk, e

$$
j_{0,1}(\eta)=\alpha-\eta(1), \quad j_{N-1, N}(\eta)=\eta(N-1)-\beta,
$$

representam a corrente microscópica nas respectivas fronteiras. Agora vamos escrever (3.2) como o gradiente discreto de uma certa função $h$. Somando e subtraindo $\eta(z) \eta(z+1)$ de (3.2) temos,

$$
\begin{aligned}
j_{z, z+1}(\eta) & =\eta(z-1) \eta(z)+\eta(z) \eta(z+1)-\eta(z-1) \eta(z+1) \\
& -\eta(z) \eta(z+1)-\eta(z+1) \eta(z+2)+\eta(z) \eta(z+2) .
\end{aligned}
$$

Em particular, utilizando mais uma vez a convenção feita em (2.1), e fazendo 
$h(\eta)=\eta(1) \eta(2)+\eta(2) \eta(3)-\eta(1) \eta(3)$, escrevemos $j_{z, z+1}(\eta)$ da seguinte forma

$$
j_{z, z+1}(\eta)=\tau_{z-2} h(\eta)-\tau_{z-1} h(\eta), \text { para } z \in\{1, \cdots, N-2\},
$$

onde $\tau_{z} h(\eta)=\eta(z+1) \eta(z+2)+\eta(z+2) \eta(z+3)-\eta(z+1) \eta(z+3)$ e a função $\tau_{z} \eta$ definida por

$$
\tau_{x} \eta(y)=\eta(x+y)
$$

é a translação de $\eta$ por $x$. Portanto, a corrente microscópica do PMM em contato com reservatórios é dada por

$$
\begin{aligned}
j_{0,1}(\eta) & =\alpha-\eta(1), \\
j_{z, z+1}(\eta) & =\tau_{z-2} h(\eta)-\tau_{z-1} h(\eta), \text { para } z \in\{1, \cdots, N-2\}, \\
j_{N-1, N}(\eta) & =\eta(N-1)-\beta
\end{aligned}
$$

onde $\tau_{z} h(\eta)=\eta(z+1) \eta(z+2)+\eta(z+2) \eta(z+3)-\eta(z+1) \eta(z+3)$.

\section{2}

\section{Limite Hidrodinâmico}

No capítulo anterior, estudamos o PMM em contato com reservatórios do ponto de vista microscópico, isto é, nosso conjunto de sítios foi definido em um espaço discreto $\{1, \cdots, N-1\}$. Agora, queremos estudar a evolução espaço/tempo da única quantidade conservada no bulk, a densidade de partículas, e para isso precisamos fazer a relação microscópica/macroscópica, isto é, queremos definir nosso conjunto de sítios no espaço contínuo $[0,1]$. Para isso, vamos fazer a identificação do espaço discreto $\{1, \cdots, N-1\}$ com o espaço contínuo $[0,1]$ através da aplicação que leva $x$ em $\frac{x}{N}$. Dessa forma, as partículas realizarão seus saltos no conjunto $\{1 / N, 2 / N, \cdots,(N-1) / N\}$. Assim, nosso objetivo será mostrar que quando $N \rightarrow \infty$, a densidade de partículas em $[0,1]$ se comporta como a solução da equação em meios porosos com condições de Dirichlet apresentada mais à frente em (3.21).

Considere o processo $\left\{\eta_{t}\right\}_{t \geq 0}$ com espaço de estados $\{0,1\}^{\Sigma_{N}}$ e com gerador infinitesimal $N^{2} L_{N}$ definido em (2.3). Pelo Lema A.8, sabemos que considerar o gerador $N^{2} L_{N}$ corresponde a tomar o processo $\left\{\eta_{t N^{2}}\right\}_{t \geq 0}$, ou seja, a escala microscópica de tempo é $t N^{2}$. Essa mudança na escala de tempo é importante para conseguirmos a convergência da densidade de partículas. Denotaremos por $C^{1,2}([0, T] \times[0,1])$ o conjunto das funções $H:[0, T] \times$ $[0,1] \longrightarrow \mathbb{R}$ tais que $H$ é de classe $C^{1}$ no tempo e de classe $C^{2}$ no espaço. Vamos escrever o subconjunto $C_{0}^{1,2}([0, T] \times[0,1])$ das funções $H \in C^{1,2}([0, T] \times[0,1])$ tais que $H(t, 0)=H(t, 1)=0$ para todo $t \in[0, T]$, e sempre que nos referirmos a uma função teste, estaremos nos referindo a uma função deste espaço. Agora, 
vamos definir a medida empírica para o processo $\left\{\eta_{t}\right\}_{t \geq 0}$. Esta medida está definida em $\{0,1\}^{\Sigma_{N}}$, e para cada $t \geq 0$ dá peso $1 / N$ a cada sítio ocupado da configuração $\eta_{t}$.

Definição 3.2. Para cada configuração $\eta \in\{0,1\}^{\Sigma_{N}}$ definimos a medida empírica $\pi^{N}(\eta, d u)$ em $[0,1]$ como

$$
\pi^{N}(\eta, d u)=\frac{1}{N} \sum_{x \in \Sigma_{N}} \eta(x) \delta_{\frac{x}{N}}(d u)
$$

onde $\delta_{a}$ é a medida Delta de Dirac em a.

Uma vez que queremos analisar a evolução temporal da medida empírica associada ao processo $\left\{\eta_{t}\right\}_{t \geq 0}$, definimos um processo de medidas empíricas da seguinte forma $\pi_{t}^{N}(\eta, d u):=\pi^{N}\left(\eta_{t N^{2}}, d u\right)$. Com esta notação, se $H \in$ $C_{0}^{1,2}([0, T] \times[0,1])$, então a integral de $H$ com respeito à medida empírica $\pi_{t}^{N}$ será dada por

$$
\left\langle\pi_{t}^{N}, H\right\rangle:=\int H(u) \pi_{t}^{N}(\eta, d u)=\frac{1}{N} \sum_{x=1}^{N-1} H_{x} \eta_{t N^{2}}(x),
$$

onde $H_{x}:=H\left(\frac{x}{N}\right)$.

Definição 3.3. O laplaciano discreto de $H$ em $\frac{x}{N} \operatorname{com} x \in \Sigma_{N}$ é dado por

$$
\Delta_{N} H_{x}=N^{2}\left\{H_{x-1}-2 H_{x}+H_{x+1}\right\} .
$$

Definimos também o gradiente discreto por

$$
\begin{aligned}
& \nabla_{N}^{+} H_{x}=N\left(H_{x+1}-H_{x}\right), \\
& \nabla_{N}^{-} H_{x}=-N\left(H_{x-1}-H_{x}\right) .
\end{aligned}
$$

Assim, calculando o gerador infinitesimal aplicado na medida empírica e utilizando as correntes microscópicas calculadas em (3.5) temos

$$
\begin{aligned}
N^{2} L_{N}\left\langle\pi_{t}^{N}, H\right\rangle & =N^{2} L_{N}\left[\frac{1}{N} \sum_{x=1}^{N-1} H_{x} \eta_{t N^{2}}(x)\right] \\
& =\frac{N^{2}}{N} \sum_{x=1}^{N-1} H_{x} L_{N} \eta_{t N^{2}}(x) \\
& =\frac{N^{2}}{N}\left[H_{1}\left(j_{0,1}\left(\eta_{t N^{2}}\right)-j_{1,2}\left(\eta_{t N^{2}}\right)\right)+H_{2}\left(j_{1,2}\left(\eta_{t N^{2}}\right)-j_{2,3}\left(\eta_{t N^{2}}\right)\right)\right. \\
& \left.+\cdots+H_{N-1}\left(j_{N-2, N-1}\left(\eta_{t N^{2}}\right)-j_{N-1, N}\left(\eta_{t N^{2}}\right)\right)\right]
\end{aligned}
$$




$$
\begin{aligned}
& =\frac{N^{2}}{N}\left[H_{1}\left(\alpha-\eta_{t N^{2}}(1)-\tau_{(-1)} h\left(\eta_{t N^{2}}\right)+h\left(\eta_{t N^{2}}\right)\right)+H_{2}\left(\tau_{(-1)} h\left(\eta_{t N^{2}}\right)\right.\right. \\
& \left.-2 h\left(\eta_{t N^{2}}\right)+\tau_{1} h\left(\eta_{t N^{2}}\right)\right)+H_{3}\left(h\left(\eta_{t N^{2}}\right)-2 \tau_{1} h\left(\eta_{t N^{2}}\right)+\tau_{2} h\left(\eta_{t N^{2}}\right)\right) \\
& \left.+\cdots+H_{N-1}\left(\tau_{N-4} h\left(\eta_{t N^{2}}\right)-\tau_{N-3} h\left(\eta_{t N^{2}}\right)-\eta_{t N^{2}}(N-1)+\beta\right)\right] \\
& =\frac{1}{N}\left[N^{2}\left(H_{0}-H_{1}+H_{2}\right) \tau_{(-1)} h\left(\eta_{t N^{2}}\right)+N^{2}\left(H_{1}-2 H_{2}+H_{3}\right) h\left(\eta_{t N^{2}}\right)\right. \\
& +N^{2}\left(H_{2}-2 H_{3}+H_{4}\right) \tau_{1} h\left(\eta_{t N^{2}}\right)+N^{2}\left(H_{3}-2 H_{4}+H_{5}\right) \tau_{2} h\left(\eta_{t N^{2}}\right) \\
& +\cdots+N^{2}\left(H_{N-3}-2 H_{N-2}+H_{N-1}\right) \tau_{N-4} h\left(\eta_{t N^{2}}\right) \\
& +N^{2}\left(H_{N-2}-H_{N-1}+H_{N}\right) \tau_{N-3} h\left(\eta_{t N^{2}}\right)+N^{2} H_{1}\left(\alpha-\eta_{t N^{2}}(1)\right) \\
& \left.+N^{2} H_{N-1}\left(\beta-\eta_{t N^{2}}(N-1)\right)\right] \\
& =\frac{1}{N} \sum_{x=2}^{N-2}\left(\Delta_{N} H_{x}\right) \tau_{x-2} h\left(\eta_{t}\right)+\frac{1}{N}\left[N^{2}\left(H_{0}-H_{1}+H_{2}\right) \tau_{(-1)} h\left(\eta_{t N^{2}}\right)\right. \\
& +N^{2}\left(H_{N-2}-H_{N-1}+H_{N}\right) \tau_{N-3} h\left(\eta_{t N^{2}}\right)+N^{2} H_{1}\left(\alpha-\eta_{t N^{2}}(1)\right) \\
& \left.+N^{2} H_{N-1}\left(\beta-\eta_{t N^{2}}(N-1)\right)\right] \\
& =\frac{1}{N} \sum_{x=2}^{N-2}\left(\Delta_{N} H_{x}\right) \tau_{x-2} h\left(\eta_{t N^{2}}\right)+\frac{1}{N}\left[N^{2}\left(H_{0}-2 H_{1}+H_{2}\right) \tau_{(-1)} h\left(\eta_{t N^{2}}\right)\right. \\
& +N^{2}\left(H_{N-2}-2 H_{N-1}+H_{N}\right) \tau_{N-3} h\left(\eta_{t N^{2}}\right)+N^{2} H_{1} \tau_{(-1)} h\left(\eta_{t N^{2}}\right) \\
& +N^{2} H_{N-1} \tau_{N-3} h\left(\eta_{t N^{2}}\right)+N^{2} H_{1}\left(\alpha-\eta_{t N^{2}}(1)\right) \\
& \left.+N^{2} H_{N-1}\left(\beta-\eta_{t N^{2}}(N-1)\right)\right] \\
& =\frac{1}{N} \sum_{x=1}^{N-1}\left(\Delta_{N} H_{x}\right) \varphi_{x}\left(\eta_{t N^{2}}\right)+\frac{1}{N}\left[N^{2} H_{1}\left(\alpha-\eta_{t N^{2}}(1)+\tau_{(-1)} h\left(\eta_{t N^{2}}\right)\right)\right. \\
& \left.+N^{2} H_{N-1}\left(\beta-\eta_{t N^{2}}(N-1)+\tau_{N-3} h\left(\eta_{t N^{2}}\right)\right)\right] \\
& =\frac{1}{N} \sum_{x=1}^{N-1}\left(\Delta_{N} H_{x}\right) \varphi_{x}\left(\eta_{t N^{2}}\right)+\nabla_{N}^{+} H_{0}\left(\alpha-\eta_{t N^{2}}(1)+\tau_{(-1)} h\left(\eta_{t N^{2}}\right)\right) \\
& -\nabla_{N}^{-} H_{N}\left(\beta-\eta_{t N^{2}}(N-1)+\tau_{N-3} h\left(\eta_{t N^{2}}\right)\right)
\end{aligned}
$$

onde $\varphi_{x}\left(\eta_{t N^{2}}\right)=\tau_{x-2} h\left(\eta_{t N^{2}}\right)$ para $x=1, \cdots, N-1$, e como $H \in C_{0}^{2}([0,1])$,

$$
\begin{aligned}
\nabla_{N}^{+} H_{0} & =N\left(H_{1}-H_{0}\right)=N H_{1} \\
\nabla_{N}^{-} H_{N} & =-N\left(H_{N-1}-H_{N}\right)=-N H_{N-1} .
\end{aligned}
$$

Logo,

$$
N^{2} L_{N}\left\langle\pi_{t}^{N}, H\right\rangle=\frac{1}{N} \sum_{x=1}^{N-1}\left(\Delta_{N} H_{x}\right) \varphi_{x}\left(\eta_{t N^{2}}\right)+\mathrm{c}_{1}(t, H)+\mathrm{c}_{N-1}(t, H),
$$


onde

$$
\begin{aligned}
\mathrm{c}_{1}(t, H)=\nabla_{N}^{+} & H_{0}\left(\alpha-\eta_{t N^{2}}(1)+\alpha \eta_{t N^{2}}(1)+\eta_{t N^{2}}(1) \eta_{t N^{2}}(2)-\alpha \eta_{t N^{2}}(2)\right), \\
\mathrm{c}_{N-1}(t, H)= & -\nabla_{N}^{-} H_{N}\left(\beta-\eta_{t N^{2}}(N-1)+\eta_{t N^{2}}(N-2) \eta_{t N^{2}}(N-1)\right. \\
& \left.+\eta_{t N^{2}}(N-1) \beta-\eta_{t N^{2}}(N-2) \beta\right) .
\end{aligned}
$$

Para continuarmos a nossa procura pela solução da equação diferencial parcial desejada, precisaremos usar o Teorema B.1 presente no Apêndice B. Para simplificar os cálculos vamos fixar uma função $H \in C_{0}^{2}([0,1])$ que não depende do tempo. Tomando a função $F\left(t, \eta_{t}\right)=\frac{1}{N} \sum_{x=1}^{N-1} H_{x} \eta_{t N^{2}}(x)$, do Teorema B.1, temos que

$$
M_{t}^{N}(H)=\left\langle\pi_{t}^{N}, H\right\rangle-\left\langle\pi_{0}^{N}, H\right\rangle-\int_{0}^{t}\left(\partial_{s}+N^{2} L_{N}\right)\left\langle\pi_{s}^{N}, H\right\rangle d s,
$$

é um martingal com respeito à filtração natural $\mathcal{F}_{t}:=\sigma\left(\eta_{s}: s \leq t\right)$. Como $F(s, \cdot)$ não depende do tempo, então $\partial_{s} F(s, \cdot)=0$. Assim, por $(3.9)$ podemos escrever o martingal (3.10) como

$$
\begin{aligned}
M_{t}^{N}(H) & =\left\langle\pi_{t}^{N}, H\right\rangle-\left\langle\pi_{0}^{N}, H\right\rangle-\int_{0}^{t} N^{2} L_{N}\left\langle\pi_{s}^{N}, H\right\rangle d s \\
& =\left\langle\pi_{t}^{N}, H\right\rangle-\left\langle\pi_{0}^{N}, H\right\rangle-\int_{0}^{t} \frac{1}{N} \sum_{x=1}^{N-1}\left(\Delta_{N} H_{x}\right) \varphi_{x}\left(\eta_{s N^{2}}\right) d s \\
& +\int_{0}^{t} \mathrm{c}_{1}(s, H)+\mathrm{c}_{N-1}(s, H) d s .
\end{aligned}
$$

Agora precisamos analisar os termos $c_{1}(s, H)$ e $c_{N-1}(s, H)$ de (3.11). Vamos começar olhando para $c_{1}(s, H)$. De fato,

$$
\int_{0}^{t} c_{1}(s, H) d s=\int_{0}^{t} \nabla_{N}^{+} H_{0}\left(\alpha-\eta_{s N^{2}}(1)+\alpha \eta_{s N^{2}}(1)+\eta_{s N^{2}}(1) \eta_{s N^{2}}(2)-\alpha \eta_{s N^{2}}(2)\right) d s .
$$

Seja $\mu_{N}$ uma medida qualquer. Se provarmos que

$$
\mathbb{E}_{\mu_{N}}\left|\int_{0}^{t}\left(\eta_{s N^{2}}(1)-\alpha\right) d s\right| \rightarrow 0
$$

quando $N \rightarrow \infty$, então poderemos trocar $\eta_{s N^{2}}(1)$ por $\alpha$ em (3.12). Nesta dissertação não iremos provar este fato (o leitor interessado pode ver estes lemas para outro modelo parecido, ou seja, os lemas 5.4 e 5.5 de [1]), isto ficará para um estudo futuro. Entretanto, se assumirmos que podemos fazer esta troca com um certo erro $e_{1}$, que vai para zero quando $N \rightarrow \infty$, e tomando a esperança com respeito a uma medida $\mu_{N}$, podemos reescrever (3.12) da seguinte forma 


$$
\begin{aligned}
\mathbb{E}_{\mu_{N}}\left[\int_{0}^{t} \mathrm{c}_{1}(s, H) d s\right] & =\mathbb{E}_{\mu_{N}}\left[\int_{0}^{t} \nabla_{N}^{+} H_{0}\left(\alpha-\alpha+\alpha^{2}+\alpha \eta_{s N^{2}}(2)-\alpha \eta_{s N^{2}}(2)\right) d s\right] \\
& +e_{1} \\
& =\int_{0}^{t} \nabla_{N}^{+} H_{0} \alpha^{2} d s+e_{1} \\
& =t \nabla_{N}^{+} H_{0} \alpha^{2}+e_{1} .
\end{aligned}
$$

De modo análogo ao anterior, se provarmos que

$$
\mathbb{E}_{\mu_{N}}\left|\int_{0}^{t}\left(\eta_{s N^{2}}(N-1)-\beta\right) d s\right| \rightarrow 0
$$

quando $N \rightarrow \infty$, poderemos trocar $\eta_{s N^{2}}(N-1)$ por $\beta$ com um certo erro $e_{N-1}$ que vai para zero quando $N \rightarrow \infty$, obtendo

$$
\mathbb{E}_{\mu_{N}}\left[\int_{0}^{t} \mathrm{c}_{N-1}(s, H) d s\right]=-t \nabla_{N}^{-} H_{N} \beta^{2} d s+e_{N-1} .
$$

Assim, com os resultados obtidos em (3.14) e (3.16), e tomando a esperança com respeito a qualquer medida $\mu_{N}$ que satisfaça (3.13) e (3.15), temos que o martingal (3.11) será dado por

$$
\begin{aligned}
\mathbb{E}_{\mu_{N}}\left[M_{t}^{N}(H)\right] & =\frac{1}{N} \sum_{x=1}^{N-1} H_{x}\left(\mathbb{E}_{\mu_{N}}\left[\eta_{t N^{2}}(x)\right]-\mathbb{E}_{\mu_{N}}\left[\eta_{0}(x)\right]\right) \\
& -\int_{0}^{t} \frac{1}{N} \sum_{x=1}^{N-1} \Delta_{N} H_{x} \mathbb{E}_{\mu_{N}}\left[\varphi_{x}\left(\eta_{s N^{2}}\right)\right] d s+t \nabla_{N}^{+} H_{0} \alpha^{2} \\
& +e_{1}-t \nabla_{N}^{-} H_{N} \beta^{2}+e_{N-1} .
\end{aligned}
$$

Note que em (3.17) estamos utilizando a linearidade da esperança e o Teorema de Fubini. Como $M_{0}^{N}(H)=0$ e a esperança de um martingal é constante, então $\mathbb{E}_{\mu_{N}}\left[M_{t}^{N}(H)\right]=\mathbb{E}_{\mu_{N}}\left[M_{0}^{N}(H)\right]=0$,

$$
\begin{aligned}
0 & =\frac{1}{N} \sum_{x=1}^{N-1} H_{x}\left(\mathbb{E}_{\mu_{N}}\left[\eta_{t N^{2}}(x)\right]-\mathbb{E}_{\mu_{N}}\left[\eta_{0}(x)\right]\right)-\int_{0}^{t} \frac{1}{N} \sum_{x=1}^{N-1} \Delta_{N} H_{x} \mathbb{E}_{\mu_{N}}\left[\varphi_{x}\left(\eta_{s N^{2}}\right)\right] d s \\
& +t \nabla_{N}^{+} H_{0} \alpha^{2}-t \nabla_{N}^{-} H_{N} \beta^{2}+e_{1}+e_{N-1} .
\end{aligned}
$$

Seja $\rho_{t}$ um perfil de densidade o qual é solução da equação diferencial parcial que procuramos e seja $\rho_{t}^{N}(x)=\mathbb{E}_{\mu_{N}}\left[\eta_{t N^{2}}(x)\right]$. Esperamos que $\rho_{t}^{N}(x)$ esteja próximo de $\rho_{t}\left(\frac{x}{N}\right)$, o qual denotaremos com a notação $\rho_{t}^{N}(x) \sim \rho_{t}\left(\frac{x}{N}\right)$. Agora, considerando $\rho_{0}^{N}(x) \sim \rho_{0}\left(\frac{x}{N}\right)$, e assumindo que a esperança do produto é o 
produto das esperanças segue que

$$
\begin{aligned}
& \mathbb{E}_{\mu_{N}}\left[\varphi_{1}\left(\eta_{t N^{2}}\right)\right]=\mathbb{E}_{\mu_{N}}\left[\tau_{(-1)} h\left(\eta_{t N^{2}}\right)\right] \\
& =\mathbb{E}_{\mu_{N}}\left[\eta_{t N^{2}}(1) \eta_{t N^{2}}(2)+\eta_{t N^{2}}(1) \alpha-\eta_{t N^{2}}(2) \alpha\right] \\
& =\mathbb{E}_{\mu_{N}}\left[\eta_{t N^{2}}(1) \eta_{t N^{2}}(2)\right]+\mathbb{E}_{\mu_{N}}\left[\alpha \eta_{t N^{2}}(1)\right]-\mathbb{E}_{\mu_{N}}\left[\alpha \eta_{t N^{2}}(2)\right] \\
& \sim \rho_{t}\left(\frac{1}{N}\right) \rho_{t}\left(\frac{2}{N}\right)+\alpha \rho_{t}\left(\frac{1}{N}\right)+\alpha \rho_{t}\left(\frac{2}{N}\right) \\
& \mathbb{E}_{\mu_{N}}\left[\varphi_{2}\left(\eta_{t N^{2}}\right)\right]=\mathbb{E}\left[\eta_{t N^{2}}(2) \eta_{t N^{2}}(1)+\eta_{t N^{2}}(3) \eta_{t N^{2}}(2)-\eta_{t N^{2}}(3) \eta_{t N^{2}}(1)\right] \\
& =\mathbb{E}_{\mu_{N}}\left[\eta_{t N^{2}}(2) \eta_{t N^{2}}(1)\right]+\mathbb{E}_{\mu_{N}}\left[\eta_{t N^{2}}(3) \eta_{t N^{2}}(2)\right] \\
& -\mathbb{E}_{\mu_{N}}\left[\eta_{t N^{2}}(3) \eta_{t N^{2}}(1)\right] \\
& \sim \rho_{t}\left(\frac{2}{N}\right) \rho_{t}\left(\frac{1}{N}\right)+\rho_{t}\left(\frac{3}{N}\right) \rho_{t}\left(\frac{2}{N}\right)-\rho_{t}\left(\frac{3}{N}\right) \rho_{t}\left(\frac{1}{N}\right) \\
& \mathbb{E}_{\mu_{N}}\left[\varphi_{x}\left(\eta_{t N^{2}}\right)\right]=\mathbb{E}_{\mu_{N}}\left[\eta_{t N^{2}}(x) \eta_{t N^{2}}(x-1)\right]+\mathbb{E}_{\mu_{N}}\left[\eta_{t N^{2}}(x+1) \eta_{t N^{2}}(x)\right] \\
& -\mathbb{E}_{\mu_{N}}\left[\eta_{t N^{2}}(x+1) \eta_{t N^{2}}(x-1)\right] \\
& \sim \rho_{t}\left(\frac{x}{N}\right) \rho_{t}\left(\frac{x-1}{N}\right)+\rho_{t}\left(\frac{x+1}{N}\right) \rho_{t}\left(\frac{x}{N}\right)-\rho_{t}\left(\frac{x+1}{N}\right) \rho_{t}\left(\frac{x-1}{N}\right) .
\end{aligned}
$$

Assumindo que

$$
\rho_{t}\left(\frac{x \pm 1}{N}\right) \sim \rho_{t}\left(\frac{x}{N}\right)
$$

podemos escrever para cada $x \in \Sigma_{N}$

$$
\mathbb{E}_{\mu_{N}}\left[\varphi_{x}\left(\eta_{t N^{2}}\right)\right] \sim \rho_{t}\left(\frac{x}{N}\right)^{2}
$$

Portanto, por (3.18) temos

$$
\begin{aligned}
0 & =\frac{1}{N} \sum_{x=1}^{N-1} H_{x}\left(\rho_{t}\left(\frac{x}{N}\right)-\rho_{0}\left(\frac{x}{N}\right)\right)-\int_{0}^{t} \frac{1}{N} \sum_{x=1}^{N-1}\left(\Delta_{N} H_{x}\right) \rho_{s}\left(\frac{x}{N}\right)^{2} d s \\
& -t \nabla_{N}^{-} H_{N} \beta^{2}+t \nabla_{N}^{+} H_{0} \alpha^{2}+e_{1}+e_{N-1} .
\end{aligned}
$$

Por fim, fazendo $N \rightarrow \infty$ e usando o fato de que $e_{1}$ e $e_{N-1}$ são negligíveis, temos

$$
\begin{aligned}
& 0=\int_{0}^{1}\left(\rho_{t}(u)-\rho_{0}(u)\right) H(u) d u-\int_{0}^{t} \int_{0}^{1} \Delta_{N} H(u)\left(\rho_{s}(u)\right)^{2} d u d s \\
& \quad-t\left(\partial_{u} H(1) \beta^{2}-\partial_{u} H(0) \alpha^{2}\right) .
\end{aligned}
$$

Na próxima seção faremos a formulação fraca da equação em meios porosos com condições de Dirichlet para uma função $H$ que depende do espaço e do tempo. Quando essa função não depender do tempo, veremos que a formulação fraca corresponderá à (3.20). 


\section{3}

\section{Equação em meios porosos}

A equação em meios porosos é uma equação diferencial parcial parabólica que aparece de maneira muito natural para descrever processos envolvendo difusão ou transferência de calor [20]. Ela é muito utilizada, por exemplo, para descrever a radiação de calor em plasmas de acordo com o espaço e o tempo. O modelo que introduzimos no Capítulo 2 está relacionado com a equação em meios porosos com condições de Dirichlet, que é dada por

$$
\begin{cases}\partial_{t} \rho(t, u)=\partial_{u}^{2} \rho^{2}(t, u), & \text { para } t>0, u \in(0,1), \\ \rho(t, 0)=\alpha, & \text { para } t>0, \\ \rho(t, 1)=\beta, & \text { para } t>0, \\ \rho(0, u)=\rho_{0}(u), & u \in[0,1],\end{cases}
$$

onde $\rho$ é uma função definida em $[0, T] \times[0,1]$ e que toma valores em $[0,1]$, e $\rho_{0}:[0,1] \rightarrow[0,1]$ é uma função mensurável.

Uma equação diferencial parcial pode ter soluções que não são diferenciáveis, são as chamadas soluções fracas. Para definir tais soluções temos que reescrever a equação diferencial parcial na chamada formulação fraca [14]. Denotaremos por $C_{c}^{\infty}(0,1)$ o espaço de todas as funções reais $\phi \in C^{\infty}$ com suporte compacto. A derivada de $H \in C^{1,2}([0, T] \times[0,1])$ será denotada por $\partial_{t} H$ (primeira variável) e $\partial_{u} H$ (segunda variável). Escreveremos $\Delta H$ para $\partial_{u}^{2} H$. O conjunto $C^{\infty}([0,1])$ denota o conjunto das funções suaves no intervalo $[0,1]$. O semi-produto interno $\langle\cdot, \cdot\rangle_{1}$ é definido no conjunto $C^{\infty}([0,1])$ por

$$
\langle G, H\rangle_{1}=\int_{0}^{1}\left(\partial_{u} G\right)(u)\left(\partial_{u} H\right)(u) d u .
$$

A semi-norma correspondente é denotada por $\|\cdot\|_{1}$.

Definição 3.4. Seja $f \in L^{2}(0,1)$. Dizemos que $f$ é fracamente diferenciável se existir $g \in L^{2}(0,1)$ tal que

$$
\int_{0}^{1} f(u) \varphi^{\prime}(u) d u=-\int_{0}^{1} g(u) \varphi(u) d u, \forall \varphi \in C_{c}^{\infty}(0,1) .
$$

Nesse caso, dizemos que $g$ é derivada fraca ou derivada generalizada de $f$ e escrevemos $g=f^{\prime}$.

Teorema 3.5. A derivada fraca de $f \in L^{2}(0,1)$, se existir, é única.

Demonstração. Suponha que existam $g, h \in L^{2}(0,1)$ tais que

$$
\int_{0}^{1} f(u) \varphi^{\prime}(u) d u=-\int_{0}^{1} g(u) \varphi(u) d u=-\int_{0}^{1} h(u) \varphi(u) d u, \forall \varphi \in C_{c}^{\infty}(0,1) .
$$


Então

$$
\int_{0}^{1}(g(u)-h(u)) \varphi(u) d u=0, \quad \forall \varphi \in C_{c}^{\infty}(0,1) .
$$

A igualdade acima significa que $(g-h)$ é ortogonal (em $\left.L^{2}\right)$ a toda função $\varphi \in C_{c}^{\infty}(0,1) \subset L^{2}(0,1)$. Como $C_{c}^{\infty}(0,1)$ é denso em $L^{2}$ (vide [4]), isso implica que $g-h$ é ortogonal a qualquer função de $L^{2}(0,1)$. Assim, $g-h=0 \mathrm{em}$ $L^{2}(0,1)$, ou seja, $g=h$.

Definição 3.6. Definimos o espaço de Sobolev $\mathcal{H}^{1}(0,1)$ como o conjunto de todas as funções $f \in L^{2}(0,1)$ que possuem derivada fraca.

Em $\mathcal{H}^{1}(0,1)$ definimos a norma $\|f\|_{\mathcal{H}^{1}(0,1)}:=\left(\|f\|_{L^{2}(0,1)}+\|f\|_{1}\right)$, que é gerada pelo produto interno $\langle f, g\rangle_{\mathcal{H}^{1}(0,1)}=\langle f, g\rangle_{L^{2}(0,1)}+\langle f, g\rangle_{1}$. O espaço $\left(\mathcal{H}^{1}(0,1),\|\cdot\|_{\mathcal{H}^{1}(0,1)}\right)$ é um espaço de Hilbert, veja [4].

Definição 3.7. O espaço $L^{2}\left(0, T ; \mathcal{H}^{1}(0,1)\right)$ é o conjunto das funções mensuráveis $f:[0, T] \rightarrow \mathcal{H}^{1}(0,1)$ tal que

$$
\int_{0}^{T}\left\|f_{t}\right\|_{\mathcal{H}^{1}(0,1)}^{2} d t<\infty
$$

Agora estamos interessados em encontrar a formulação fraca da equação em meios porosos com condições de Dirichlet. Para isso vamos fazer uma formulação heurística. Consideramos a equação (3.21) e fixamos um tempo $T>0$ e $H \in C_{0}^{1,2}([0, T] \times[0,1])$. Multiplicamos ambos os membros da equação $\partial_{t} \rho(t, u)=\partial_{u}^{2} \rho^{2}(t, u)$ por $H(t, u)$, e integrando ambos os membros dessa equação sobre a região $[0, T] \times[0,1]$ obtemos:

$$
\underbrace{\int_{0}^{1} \int_{0}^{t} \partial_{s} \rho(s, u) H(s, u) d s d u}_{=I_{1}}=\underbrace{\int_{0}^{1} \int_{0}^{t} \partial_{u}^{2} \rho^{2}(s, u) H(s, u) d s d u}_{=I_{2}} .
$$

Integrando $I_{1}$ por partes temos

$$
I_{1}=\int_{0}^{1}(\rho(t, u) H(t, u)-\rho(0, u) H(0, u)) d u-\int_{0}^{1} \int_{0}^{t} \rho(s, u) \partial_{s} H(s, u) d s d u
$$

Utilizando o Teorema de Fubini e fazendo integração por partes duas vezes, temos

$$
\begin{aligned}
l_{2} & =\int_{0}^{t} \partial_{u} \rho^{2}(s, 1) \underbrace{H(s, 1)}_{=0}-\partial_{u} \rho^{2}(s, 0) \underbrace{H(s, 0)}_{=0} d s-\int_{0}^{t} \int_{0}^{1} \partial_{u} \rho^{2}(s, u) \partial_{u} H(s, u) d u d s \\
& =-\int_{0}^{t} \int_{0}^{1} \partial_{u} \rho^{2}(s, u) \partial_{u} H(s, u) d u d s=-\int_{0}^{t}\left[\rho^{2}(s, 1) \partial_{u} H(s, 1)\right. \\
& \left.-\rho^{2}(s, 0) \partial_{u} H(s, 0) d s\right]+\int_{0}^{t} \int_{0}^{1} \rho^{2}(s, u) \partial_{u}^{2} H(s, u) d u d s .
\end{aligned}
$$


Como $\rho(t, 0)=\alpha, \rho(t, 1)=\beta$ e $H \in C_{0}^{1,2}([0, T] \times[0,1]),(3.23)$ pode ser escrita como

$$
\begin{aligned}
& \int_{0}^{1} \rho(t, u) H(t, u)-\rho(0, u) H(0, u) d u-\int_{0}^{1} \int_{0}^{t} \rho(s, u) \partial_{s} H(s, u) d s d u \\
& =-\int_{0}^{t} \beta^{2} \partial_{u} H(s, 1)-\alpha^{2} \partial_{u} H(s, 0) d s+\int_{0}^{t} \int_{0}^{1} \rho^{2}(s, u) \partial_{u}^{2} H(s, u) d u d s
\end{aligned}
$$

para todo $t \in[0, T]$.

Definição 3.8. Seja $\rho_{0}:[0,1] \rightarrow \mathbb{R}$ uma função mensurável. Dizemos que uma função não-negativa $\rho:[0, T] \times[0,1] \rightarrow[0,1]$ é uma solução fraca da equação (3.21) se

(i) $\rho^{2} \in L^{2}\left(0, T: \mathcal{H}^{1}(0,1)\right)$;

(ii) $\rho$ satisfaz a identidade

$$
\begin{aligned}
& \int_{0}^{1}(\rho(t, u) H(t, u)-\rho(0, u) H(0, u)) d u-\int_{0}^{1} \int_{0}^{t} \rho(s, u) \partial_{s} H(s, u) d s d u \\
& =\int_{0}^{1} \int_{0}^{t} \rho^{2}(s, u) \partial_{u}^{2} H(s, u) d s d u-\int_{0}^{t}\left(\beta^{2} \partial_{u} H(s, 1)-\alpha^{2} \partial_{u} H(s, 0)\right) d s
\end{aligned}
$$

para toda $H \in C_{0}^{1,2}([0, T] \times[0,1])$ e todo $t \in[0, T]$;

(iii) $\rho(t, 0)=\alpha$ e $\rho(t, 1)=\beta$ para todo $t \in[0, T]$.

Teorema 3.9. Se existe uma solução fraca da equação em meios porosos com condições de Dirichlet dada em (3.21), então esta solução é única.

Demonstração. Suponha que $\rho_{1}$ e $\rho_{2}$ são soluções fracas de (3.21) partindo da mesma condição inicial. Então, fazendo somente uma integração por partes em (3.23) temos que

$$
\begin{aligned}
& \int_{0}^{1}\left(\rho_{1}(t, u)-\rho_{2}(t, u)\right) H(t, u) d u-\int_{0}^{1} \underbrace{\left(\rho_{1}(0, u)-\rho_{2}(0, u)\right)}_{=0} H(0, u) d u \\
& +\int_{0}^{1} \int_{0}^{t}\left(\partial_{u} \rho_{1}^{2}(s, u)-\partial_{u} \rho_{2}^{2}(s, u)\right) \partial_{u} H(s, u) d s d u \\
& -\int_{0}^{1} \int_{0}^{t}\left(\rho_{1}(s, u)-\rho_{2}(s, u)\right) \partial_{s} H(s, u) d s d u=0
\end{aligned}
$$

para toda função teste $H \in C_{0}^{1,2}([0, T] \times[0,1])$. Assim, consideremos a seguinte função teste

$$
\zeta(t, u)= \begin{cases}\int_{t}^{T}\left(\rho_{1}^{2}(r, u)-\rho_{2}^{2}(r, u)\right) d r, & \text { se } 0<t<T \\ 0, & \text { se } t \geq T\end{cases}
$$


onde $T>0$. Observe pelo item $i i i)$ da Definição 3.8 que $\zeta(t, 0)=\zeta(t, 1)=0$. Assim, por simplicidade, suponha que $\zeta \in C_{0}^{1,2}([0, T] \times[0,1])$ (para mais detalhes vide [20]). Agora, de (3.25) segue que

$$
\begin{aligned}
& \partial_{t} \zeta(t, u)=-\left(\rho_{1}^{2}(t, u)-\rho_{2}^{2}(t, u)\right) \in L^{2}([0, T] \times[0,1]) \\
& \partial_{u} \zeta(t, u)=\int_{t}^{T}\left(\partial_{u} \rho_{1}^{2}(r, u)-\partial_{u} \rho_{2}^{2}(r, u)\right) d r \in L^{2}([0, T] \times[0,1]) .
\end{aligned}
$$

Consequentemente substituindo $H$ por $\zeta$ em (3.24), temos

$$
\begin{aligned}
& \int_{0}^{1}\left(\rho_{1}(t, u)-\rho_{2}(t, u)\right) \underbrace{\zeta(t, u)}_{=0} d u+\int_{0}^{t} \int_{0}^{1}\left(\partial_{u} \rho_{1}^{2}(s, u)-\partial_{u} \rho_{2}^{2}(s, u)\right) \partial_{u} \zeta(s, u) d u d s \\
& -\int_{0}^{t} \int_{0}^{1}\left(\rho_{1}(s, u)-\rho_{2}(s, u)\right) \partial_{s} \zeta(s, u) d u d s=0 .
\end{aligned}
$$

Portanto, usando (3.26) segue que

$$
\begin{aligned}
& \int_{0}^{1} \int_{0}^{t}\left\{\left(\rho_{1}(s, u)-\rho_{2}(s, u)\right)\left(\rho_{1}^{2}(s, u)-\rho_{2}^{2}(s, u)\right)\right. \\
& \left.+\left(\partial_{u} \rho_{1}^{2}(s, u)-\partial_{u} \rho_{2}^{2}(s, u)\right)\left(\int_{t}^{T}\left(\partial_{u} \rho_{1}^{2}(r, u)-\partial_{u} \rho_{2}^{2}(r, u)\right) d r\right)\right\} d s d u=0 \\
& \Leftrightarrow \int_{0}^{1} \int_{0}^{t}\left\{\left(\rho_{1}(s, u)-\rho_{2}(s, u)\right)^{2}\left(\rho_{1}(s, u)+\rho_{2}(s, u)\right)\right. \\
& +\underbrace{\left(\partial_{u} \rho_{1}^{2}(s, u)-\partial_{u} \rho_{2}^{2}(s, u)\right)}_{=g(s)} \underbrace{\left(\int_{t}^{T}\left(\partial_{u} \rho_{1}^{2}(r, u)-\partial_{u} \rho_{2}^{2}(r, u)\right) d r\right)}_{\int_{t}^{T} g(r) d t}\} d s d u=0 \\
& \Leftrightarrow \int_{0}^{1} \int_{0}^{t}\left\{\left(\rho_{1}(s, u)-\rho_{2}(s, u)\right)^{2}\left(\rho_{1}(s, u)+\rho_{2}(s, u)\right) d s d u\right. \\
& +\int_{0}^{1} \underbrace{\left(\int_{0}^{t} \int_{0}^{t} g(s) g(r) \mathbb{1}_{s \leq r \leq t} d r d s\right)}_{=G} d u=0 .
\end{aligned}
$$

Como

$$
\left(\int_{0}^{t} g(r) d r\right)^{2}=\int_{0}^{t} \int_{0}^{t} g(r) g(s) \mathbb{1}_{s \leq r \leq t} d r d s+\int_{0}^{t} \int_{0}^{t} g(r) g(s) \mathbb{1}_{t \geq s \geq r} d r d s
$$

podemos escrever (3.3) como

$$
\begin{aligned}
\int_{0}^{1} \int_{0}^{t} & \left\{\left(\rho_{1}(s, u)-\rho_{2}(s, u)\right)^{2}\left(\rho_{1}(s, u)+\rho_{2}(s, u)\right) d s d u\right. \\
+ & \frac{1}{2} \int_{0}^{1}\left(\int_{0}^{t}\left(\partial_{u} \rho_{1}^{2}(r, u)-\partial_{u} \rho_{2}^{2}(r, u)\right) d r\right)^{2} d u=0 .
\end{aligned}
$$

Portanto, como os integrandos em (3.27) são não-negativos e a soma das integrais se anula, concluímos que $\rho_{1}(t, u)=\rho_{2}(t, u)$ quase certamente em $[0, T] \times[0,1]$. 


\section{4}

\section{Estados estacionários fora do equilíbrio}

Este capítulo tem como objetivo dar uma visão geral sobre o SSEP em contato com reservatórios e sobre estados estacionários fora do equilíbrio. Dividimos este capítulo em duas Seções. Na Seção 4.1, faremos a construção do SSEP em contato com reservatórios via gerador infinitesimal e calcularemos sua corrente microscópica. Por fim, na Seção 4.2, falaremos sobre sistemas em equilíbrio e fora do equilíbrio, e calcularemos o perfil de densidade e a evolução temporal do perfil para o SSEP em contato com reservatórios.

\section{1}

\section{SSEP em contato com reservatórios}

No Capítulo 2 fizemos uma definição para o SSEP em contato com reservatórios. Agora, vamos defini-lo formalmente via gerador infinitesimal. Fixando $\alpha, \beta \in(0,1)$, definimos o SSEP em contato com reservatórios como um processo de Markov $\left\{\eta_{t}\right\}_{t \geq 0}$ com espaço de estados $\Omega_{N}=\{0,1\}^{\Sigma_{N}}$ onde $\Sigma_{N}=\{1, \cdots, N-1\}$, o qual pode ser descrito em termos do seu gerador infinitesimal $\tilde{L}_{N}$ agindo sobre uma função $f: \Omega_{N} \longrightarrow \mathbb{R}$ como

$$
\left(\tilde{L}_{N} f\right)(\eta)=\left(\tilde{L}_{N, b u l k} f\right)(\eta)+\left(\tilde{L}_{N, b} f\right)(\eta)
$$

onde o gerador infinitesimal da dinâmica no bulk é dado por

$$
\left(\tilde{L}_{N, b u l k} f\right)(\eta)=\sum_{x=1}^{N-2}\left[f\left(\eta^{x, x+1}\right)-f(\eta)\right]
$$

e o gerador infinitesimal da dinâmica na fronteira é o mesmo que em (2.3).

Exemplo 4.1. Considere $z \in \Sigma_{N}$ e $f_{z}: \Omega_{N} \longrightarrow\{0,1\}$ definida por $f_{z}(\eta)=$ $\eta(z)$. Calculemos $\left(\tilde{L}_{N} f_{z}\right)(\eta)$. Como no PMM em contato com reservatórios, a seguir calcularemos a corrente microscópica para o SSEP em contato com reservatórios.

No caso $z \in\{2, \cdots, N-2\}$ temos

$$
\begin{aligned}
\tilde{L}_{N, b u l k} \eta(z) & =\sum_{x=1}^{N-2}\left\{\eta^{x, x+1}(z)-\eta(z)\right\} \\
& =\eta(z-1)-\eta(z)+\eta(z+1)-\eta(z)
\end{aligned}
$$




$$
=\eta(z-1)-2 \eta(z)+\eta(z+1) .
$$

No caso $z=1$ temos $\tilde{L}_{N, b u l k} \eta(1)=\sum_{x=1}^{N-2}\left\{\eta^{x, x+1}(1)-\eta(1)\right\}=\eta(2)-\eta(1)$. Similarmente, se $z=N-1$ temos $\tilde{L}_{N, b u l k} \eta(N-1)=\eta(N-2)-\eta(N-1)$. Assim, por (4.1) e pelos cálculos apresentados no Exemplo 3.1 para o gerador na fronteira, temos que

$$
\begin{aligned}
\tilde{L}_{N} \eta(1) & =\alpha-2 \eta(1)+\eta(2), \\
\tilde{L}_{N} \eta(z) & =\eta(z-1)-2 \eta(z)+\eta(z+1) \text { para } z \in\{2, \cdots, N-2\}, \\
\tilde{L}_{N} \eta(N-1) & =\eta(N-2)-2 \eta(N-1)+\beta
\end{aligned}
$$

Portanto, usando a convenção (2.1) temos

$$
\begin{aligned}
\tilde{L}_{N} \eta(z) & =\eta(z-1)-2 \eta(z)+\eta(z+1) \\
& =\underbrace{(\eta(z-1)-\eta(z))}_{j_{z-1, z}(\eta)}+\underbrace{(\eta(z+1)-\eta(z))}_{j_{z, z+1}(\eta)}, \forall z \in \Sigma_{N}
\end{aligned}
$$

onde $j_{z, z+1}(\eta)$ é a diferença entre a taxa de salto de $z$ para $z+1$ e a taxa de salto de $z+1$ para $z$, que representa a corrente microscópica associada ao elo $\{z, z+1\}$.

\section{2}

\section{Sistemas em equilíbrio e fora do equilíbrio}

Com as definições apresentadas acima em mente, nossa ideia agora será analisar o que acontece com o sistema à medida que o tempo evolui. Podemos dizer que temos duas correntes de partículas no SSEP em contato com reservatórios, uma corrente externa que vem dos reservatórios, e uma corrente vinda diretamente da dinâmica das partículas do bulk. Como dito anteriormente no Capítulo 2, Seção 2.2, temos dois reservatórios de partículas nas fronteiras onde as partículas podem entrar para o sítio 1 com taxa $\alpha$ (respectivamente para o sítio $N-1 \mathrm{com}$ taxa $\beta$ ), e podem sair do sítio $1 \mathrm{com}$ taxa $(1-\alpha)$ (respectivamente do sítio $N-1$ com taxa $(1-\beta)$ ), respeitando a regra de exclusão. Dessa maneira, podem surgir algumas perguntas no que diz respeito à densidade de partículas do nosso sistema, por exemplo: como as partículas vão se comportar se $\alpha$ for muito próximo de 1 e $\beta$ muito próximo de 0 ? E se eles forem iguais, como as partículas se comportarão?

Ora, se $\alpha$ estiver muito próximo de 0 e $\beta$ muito próximo de 1 , quer dizer que estão entrando muitas partículas do lado direito do sistema e saindo muitas partículas do lado esquerdo do sistema. Desta forma, os reservatórios estão criando uma espécie de assimetria no sistema, isto é, quando $\alpha \neq \beta$ os reservatórios induzem uma corrente externa de partículas no sistema, mas no 
bulk, como a taxa de salto é simétrica, não existe uma direção privilegiada para o salto das partículas e, dizemos que a corrente gerada pela dinâmica no bulk é zero. Se $\alpha$ for igual a $\beta$, então os reservatórios não criam uma corrente externa pois a densidade de partículas em cada um dos reservatórios é a mesma.

$\mathrm{Na}$ física, quando os reservatórios não criam uma corrente em um determinado sistema de partículas, o sistema é chamado de sistema em equilíbrio. Caso contrário, ele é chamado de sistema fora do equilíbrio. Nosso propósito para esta Seção é caracterizar as medidas estacionárias para o SSEP em contato com reservatórios em ambos os casos, em equilíbrio $(\alpha=\beta)$ e fora do equilíbrio $(\alpha \neq \beta)$. Com esta caracterização, podemos verificar facilmente utilizando a Proposição 2.6 que a única medida estacionária do SSEP no equilíbrio é a medida Bernoulli produto. Por outro lado, um dos grandes problemas de sistema de partículas é saber quais são as medidas estacionárias do sistema fora do equilíbrio, por isso nosso interesse em estudá-las.

Para o SSEP em contato com reservatórios, o cálculo da densidade ou das funções de correlação podem ser feitos diretamente da definição do modelo. Ao longo do texto denotaremos por $\left\langle\eta_{t}(x)\right\rangle_{\mu}^{N}$ a densidade de partículas no sítio $x$ no tempo $t$ com respeito a uma medida de probabilidade inicial qualquer $\mu$, a qual coincide com $\rho_{t}^{N}(x)$ introduzido no capítulo anterior. Quando estiver claro qual o valor de $N$ que estamos considerando, podemos omiti-lo escrevendo $\left\langle\eta_{t}(x)\right\rangle_{\mu}$ para a densidade de partículas. Para calcular esta densidade, vamos analisar para a evolução temporal da mesma, que é dada por

$$
\begin{aligned}
\frac{d}{d t}\left\langle\eta_{t}(1)\right\rangle_{\mu} & =\left\langle\tilde{L} \eta_{t}(1)\right\rangle_{\mu}=\left\langle\alpha-2 \eta_{t}(1)+\eta_{t}(2)\right\rangle_{\mu}=\alpha-2\left\langle\eta_{t}(1)\right\rangle_{\mu}+\left\langle\eta_{t}(2)\right\rangle_{\mu}, \\
\frac{d}{d t}\left\langle\eta_{t}(x)\right\rangle_{\mu} & =\left\langle\tilde{L} \eta_{t}(x)\right\rangle_{\mu}=\left\langle\eta_{t}(x-1)-2 \eta_{t}(x)+\eta_{t}(x+1)\right\rangle_{\mu} \\
& =\left\langle\eta_{t}(x-1)\right\rangle_{\mu}-2\left\langle\eta_{t}(x)\right\rangle_{\mu}+\left\langle\eta_{t}(x+1)\right\rangle_{\mu} \text { para } 2 \leq x \leq N-2, \\
\frac{d}{d t}\left\langle\eta_{t}(N-1)\right\rangle_{\mu} & =\left\langle\tilde{L} \eta_{t}(N-1)\right\rangle_{\mu}=\left\langle\eta_{t}(N-2)-2 \eta_{t}(N-1)+\beta\right\rangle_{\mu} \\
& =\left\langle\eta_{t}(N-2)\right\rangle_{\mu}-2\left\langle\eta_{t}(N-1)\right\rangle_{\mu}+\beta,
\end{aligned}
$$

onde para obtermos a igualdade $\frac{d}{d t}\left\langle\eta_{t}(x)\right\rangle_{\mu}=\left\langle\tilde{L} \eta_{t}(x)\right\rangle_{\mu}$ para cada $x$ em (4.4) usamos (A.7), que está no Apêndice A. Já a segunda igualdade vem dos cálculos efetuados no Exemplo 4.1. Cada expressão em (4.4) nos diz qual é o tempo médio de ocupação de cada sítio do nosso sistema. Assim, estamos interessados agora em calcular a densidade de partículas do sistema no estado estacionário, ou seja, quando a média ocupacional em cada sítio $x$ não evolui com o tempo. Para isso, sendo $\mu_{s s}$ a medida estacionária e fazendo $\rho^{N}(x)=\left\langle\eta_{t}(x)\right\rangle_{\mu_{s s}}$, o problema em (4.4) reduz-se a verificar que $\rho^{N}$ é solução da equação discreta 
dada por

$$
\left\{\begin{array}{cc}
\Delta_{N} \rho^{N}(x)=0 & \text { para } x \in \Sigma_{N}, \\
\rho^{N}(0)=\alpha, & \rho^{N}(N)=\beta,
\end{array}\right.
$$

onde definimos aqui o laplaciano discreto de $\rho^{N}$ em $x \in \Sigma_{N}$ como

$$
\Delta_{N} \rho^{N}(x)=\rho^{N}(x-1)-2 \rho^{N}(x)+\rho^{N}(x+1) .
$$

Para encontrar a solução de (4.5) basta resolver o sistema linear representado pela matriz de ordem $(N-1) \times(N+1)$ dada abaixo

$$
\left[\begin{array}{ccccccc}
1 & -2 & 1 & 0 & 0 & \cdots & 0 \\
0 & 1 & -2 & 1 & 0 & \cdots & 0 \\
& \ddots & \ddots & \ddots & \ddots & \ddots & \\
0 & \cdots & 0 & 1 & -2 & 1 & 0 \\
0 & \cdots & 0 & 0 & 1 & -2 & 1
\end{array}\right]\left[\begin{array}{c}
\alpha \\
\rho^{N}(1) \\
\vdots \\
\rho^{N}(N-1) \\
\beta
\end{array}\right]=\left[\begin{array}{c}
0 \\
0 \\
\vdots \\
0 \\
0
\end{array}\right] .
$$

Utilizando a eliminação de Gauss em (4.7) temos

$$
\left[\begin{array}{ccccccc}
1 & 0 & 0 & \cdots & 0 & -(N) & N-1 \\
0 & 1 & 0 & \cdots & 0 & -(N-1) & N-2 \\
0 & 0 & 1 & \cdots & 0 & -(N-2) & N-3 \\
\vdots & & & \ddots & \vdots & \vdots \\
0 & 0 & 0 & \cdots & 1 & -2 & 1
\end{array}\right]\left[\begin{array}{c}
\alpha \\
\rho^{N}(1) \\
\vdots \\
\rho^{N}(N-1) \\
\beta
\end{array}\right]=\left[\begin{array}{c}
0 \\
0 \\
\vdots \\
0 \\
0
\end{array}\right]
$$

Assim,

$$
\alpha-N \rho^{N}(N-1)+(N-1) \beta=0 \Longleftrightarrow \rho^{N}(N-1)=\frac{\alpha+\beta(N-1)}{N} .
$$

Logo,

$$
\begin{aligned}
\rho^{N}(1) & =(N-1) \rho^{N}(N-1)-\beta(N-2)=\frac{\alpha(N-1)+\beta}{N}, \\
\rho^{N}(2) & =(N-2) \rho^{N}(N-1)-\beta(N-3)=\frac{\alpha(N-2)+2 \beta}{\beta}, \\
& \vdots \\
\rho^{N}(N-2) & =\frac{2 \alpha+(N-2) \beta}{N} .
\end{aligned}
$$

Portanto o perfil de densidade microscópica é dado por

$$
\rho^{N}(x)=\frac{\alpha(N-x)+\beta x}{N} \text { para } 1 \leq x \leq N-1,
$$

onde $\alpha$ e $\beta$ são as densidades dos reservatórios. Para calcular a densidade macroscópica basta introduzir uma coordenada macroscópica $u=\frac{x}{N-1}$, assim, no caso macroscópico (4.8) torna-se 


$$
\begin{aligned}
\rho^{N}(u(N-1)) & =\frac{\alpha(N-(N-1) u)+\beta(N-1) u}{N} \\
& =\alpha-\alpha u+\frac{\alpha u}{N}+\beta u-\frac{\beta u}{N} .
\end{aligned}
$$

Note que para $N$ grande, (4.9) é dada por

$$
\rho^{N}(x) \sim \rho^{*}(u)=(1-u) \alpha+u \beta,
$$

e $\rho^{N}(1) \rightarrow \alpha$ e $\rho^{N}(N-1) \rightarrow \beta$ quando $N \rightarrow \infty$. Para calcularmos a corrente média do sistema no estado estacionário, lembremos de (4.3) que, dada uma configuração $\eta \in\{0,1\}^{\Sigma_{N}}$ a corrente microscópica entre os sítios $x$ e $x+1$ no SSEP é dada por

$$
j_{x, x+1}(\eta)=\eta(x)(1-\eta(x+1))-\eta(x+1)(1-\eta(x))
$$

Assim, a corrente média entre os sítios $x$ e $x+1$ com respeito a medida estacionária $\mu_{s s}$ é dada por

$$
\begin{aligned}
\left\langle j_{x, x+1}(\eta)\right\rangle_{\mu_{s s}} & =\langle\eta(x)(1-\eta(x+1))-\eta(x+1)(1-\eta(x))\rangle_{\mu_{s s}} \\
& =\langle\eta(x)\rangle_{\mu_{s s}}-\langle\eta(x+1)\rangle_{\mu_{s s}} \\
& =\frac{\alpha(N-x)+\beta x}{N}-\frac{\alpha(N-(x+1))+\beta(x+1)}{N} \\
& =\frac{\alpha-\beta}{N} .
\end{aligned}
$$

Observação 4.2. Observemos no cálculo acima que a corrente média no estado estacionário independe de $x$.

Agora estamos interessados em calcular funções de correlação de ordens superiores. A ideia aqui seria continuar com a mesma abordagem exposta anteriormente para calcular essas funções de correlação, porém, resolver essas equações da mesma forma que fizemos acima torna-se uma tarefa trabalhosa (o leitor interessado pode ver o cálculo de $\langle\eta(x) \eta(y)\rangle_{\mu}$ feito em [13]).

Assim, no próximo capítulo estudaremos o método da matriz ansatz, que nos dá um procedimento algébrico para calcular essas funções de correlação de uma maneira mais fácil do que a utilizada anteriormente. 


\section{Matriz ansatz}

Neste capítulo trabalharemos com o método da representação matricial, a chamada "matriz ansatz", que será utilizado para caracterizar as medidas estacionárias de sistemas de partículas fora do equilíbrio, como pode ser visto em $[3,5,6]$. Por ser um modelo clássico na literatura de sistema de partículas, escolhemos o processo de exclusão simples assimétrico (ASEP) em contato com reservatórios (definido no Capítulo 2) para apresentar as técnicas do método. Dividimos este capítulo em quatro Seções. Na Seção 5.1, apresentamos a definição da master equation. Na Seção 5.2, explicamos como representar os pesos das configurações do ASEP com respeito ao estado estacionário como um produto de matrizes, e provamos que esta formulação matricial nos fornece a distribuição estacionária do processo. Já na Seção 5.3, apresentamos as propriedades para as matrizes obtidas na representação matricial e, na Seção 5.4, apresentamos exemplos para estas matrizes. Esta abordagem servirá de motivação para, no próximo capítulo, obtermos informações sobre a distribuição estacionária do SSEP em contato com reservatórios, do PMM em contato com reservatórios, e por fim, do SSEP em contato com reservatórios lentos.

\section{1}

\section{A master equation}

Para preparar o leitor, em vez de começarmos esta Seção com uma abordagem sobre a matriz ansatz, começaremos definindo o que é uma master equation. A evolução de um sistema com dinâmicas estocásticas dadas por um processo de Markov é especificada por uma matriz de transição $W\left(C^{\prime}, C\right)$, que representa a taxa na qual o sistema muda de uma configuração $C^{\prime}$ para uma configuração $C$. A probabilidade $P_{t}(C)$ de encontrar o sistema em uma configuração $C$ no tempo $t$ evolui de acordo com a equação abaixo

$$
\frac{d P_{t}(C)}{d t}=\sum_{C^{\prime} \neq C} \underbrace{P_{t}\left(C^{\prime}\right) W\left(C^{\prime}, C\right)}_{\text {ganho }}-\sum_{C^{\prime} \neq C} \underbrace{P_{t}(C) W\left(C, C^{\prime}\right)}_{\text {perda }},
$$

à qual chamamos master equation.

Exemplo 5.1. Para ilustrar a definição de master equation acima, usando (5.1) e o Exemplo 2.2, vamos calcular agora $\frac{d P_{t}\left(\eta_{2}\right)}{d t}$ para o PMM em contato 
com reservatórios com espaço de estados $\{0,1\}^{\Sigma_{4}}$.

De fato, por (5.1) e observando que $W(\eta, \xi)$ corresponde ao $Q_{N}(\eta, \xi)$ do Exemplo 2.2 temos

$$
\begin{aligned}
& \frac{d P_{t}\left(\eta_{2}\right)}{d t}=P_{t}\left(\eta_{1}\right) \underbrace{W\left(\eta_{1}, \eta_{2}\right)}_{=\alpha}+P_{t}\left(\eta_{3}\right) \underbrace{W\left(\eta_{3}, \eta_{2}\right)}_{=\alpha}+P_{t}\left(\eta_{4}\right) \underbrace{W\left(\eta_{4}, \eta_{2}\right)}_{=0} \\
& +P_{t}\left(\eta_{5}\right) \underbrace{W\left(\eta_{5}, \eta_{2}\right)}_{=0}+P_{t}\left(\eta_{6}\right) \underbrace{W\left(\eta_{6}, \eta_{2}\right)}_{=1-\beta}+P_{t}\left(\eta_{7}\right) \underbrace{W\left(\eta_{7}, \eta_{2}\right)}_{=0} \\
& +P_{t}\left(\eta_{8}\right) \underbrace{W\left(\eta_{8}, \eta_{2}\right)}_{=0}-P_{t}\left(\eta_{2}\right)(\underbrace{W\left(\eta_{2}, \eta_{1}\right)}_{=1-\alpha}+\underbrace{W\left(\eta_{2}, \eta_{3}\right)}_{=\alpha}+\underbrace{W\left(\eta_{2}, \eta_{4}\right)}_{=0} \\
& +\underbrace{W\left(\eta_{2}, \eta_{5}\right)}_{=0}+\underbrace{W\left(\eta_{2}, \eta_{6}\right)}_{=\beta}+\underbrace{W\left(\eta_{2}, \eta_{7}\right)}_{=0}+\underbrace{W\left(\eta_{2}, \eta_{8}\right)}_{=0}) \\
& =P_{t}\left(\eta_{1}\right) \alpha+P_{t}\left(\eta_{3}\right) \alpha+P_{t}\left(\eta_{6}\right)(1-\beta)-P_{t}\left(\eta_{2}\right)(1+\beta) .
\end{aligned}
$$

Como dito anteriormente, estamos interessados em estudar os estados estacionários do nosso sistema de partículas, ou seja, estudar exatamente o caso onde temos um equilíbrio entre os termos de ganho e perda de (5.1). Neste caso, a solução da master equation não depende do tempo $t$, e denotaremos as soluções de (5.1) por $P(C)$. Sabemos por [15] que se $\left\{\eta_{t}\right\}_{t \geq 0}$ é um processo de Markov irredutível definido em um espaço de estados finito, então ele tem a propriedade de ser ergódico, isto é, um único estado estacionário é atingido a partir de qualquer condição inicial. Assim, uma vez que a distribuição de probabilidade $P(C)$ é única para este tipo de processo, vamos nos concentrar sobre a forma como ela é obtida analiticamente e como os valores esperados dos observáveis são calculados. Na verdade, estamos interessados na estrutura de produto matricial que essa distribuição pode ser representada quando o sistema se encontra no estado estacionário fora do equilíbrio, como será visto a seguir.

\section{2}

\section{A matriz ansatz}

Nossa abordagem para descrever o estado estacionário do ASEP em contato com reservatórios é motivada pelas técnicas apresentadas em $[3,5,6]$. A ideia consiste em escrever o peso $f_{N-1}(\eta(1), \cdots, \eta(N-1))$ da configuração $(\eta(1), \cdots, \eta(N-1))^{1}$ no estado estacionário como

$$
f_{N-1}(\eta(1), \eta(2), \cdots, \eta(N-1))=\mathbf{w}^{T} X_{\eta(1)} X_{\eta(2)} \cdots X_{\eta(N-1)} \mathbf{v},
$$

onde

${ }^{1}$ Indexamos $f$ em $N-1$ para notar que temos $N-1$ sítios. Para tornar a leitura mais simples, às vezes vamos denotar uma configuração $\eta \in\{0,1\}^{\Sigma_{N}}$ por $(\eta(1), \cdots, \eta(N-1))$ 


$$
X_{\eta(x)}=\eta(x) D+(1-\eta(x)) E,
$$

$D$ e $E$ são matrizes, e os vetores $\mathbf{w}^{T}$ e $\mathbf{v}$ realizam a conversão necessária do produto matricial para um escalar. A figura abaixo ilustra essa representação.

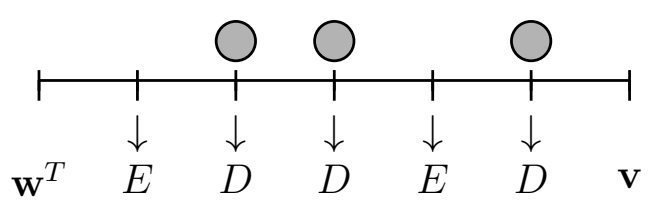

Figura 5.1: O procedimento pelo qual uma configuração de partículas é representada por um produto de matrizes.

Em geral, uma vez que as matrizes $D$ e $E$ não comutam $^{2}$, o peso $f_{N-1}(\eta(1), \cdots, \eta(N-1))$ da configuração $(\eta(1), \cdots, \eta(N-1))$ é uma função complicada. Como geralmente esse peso $f_{N-1}(\eta(1), \cdots, \eta(N-1))$ não é normalizado, a probabilidade $P(\eta(1), \eta(2), \cdots, \eta(N-1))$ de uma configuração $(\eta(1), \cdots, \eta(N-1))$ com respeito ao estado estacionário é dada por

$$
P(\eta(1), \eta(2), \cdots, \eta(N-1))=\frac{f_{N-1}(\eta(1), \eta(2), \cdots, \eta(N-1))}{Z_{N-1}},
$$

onde $Z_{N-1}$ é a soma dos pesos das $2^{N-1}$ possíveis configurações em $\{0,1\}^{\Sigma_{N}}$, isto é,

$$
Z_{N-1}=\sum_{\eta(1) \in\{0,1\}} \cdots \sum_{\eta(N-1) \in\{0,1\}} f_{N-1}(\eta(1), \eta(2), \cdots, \eta(N-1)) .
$$

Por (5.3) podemos escrever (5.5) como

$$
P(\eta(1), \eta(2), \cdots, \eta(N-1))=\frac{\mathbf{w}^{T} X_{\eta(1)} X_{\eta(2)} \cdots X_{\eta(N-1)} \mathbf{v}}{Z_{N-1}},
$$

e a normalização em (5.6) poderá ser escrita como

$$
\begin{aligned}
Z_{N-1} & =\sum_{\eta(1) \in\{1,0\}} \cdots \sum_{\eta(N-1) \in\{1,0\}} \mathbf{w}^{T} X_{\eta(1)} X_{\eta(2)} \cdots X_{\eta(N-1)} \mathbf{v} \\
& =\sum_{\eta(1) \in\{1,0\}} \cdots \sum_{\eta(N-2) \in\{1,0\}} \mathbf{w}^{T} X_{\eta(1)} X_{\eta(2)} \cdots X_{\eta(N-2)}(D+E) \mathbf{v} \\
& =\sum_{\eta(1) \in\{1,0\}} \cdots \sum_{\eta(N-3) \in\{1,0\}} \mathbf{w}^{T} X_{\eta(1)} X_{\eta(2)} \cdots X_{\eta(N-3)}(D+E)(D+E) \mathbf{v} \\
& =\cdots=\mathbf{w}^{T}(D+E)^{N-1} \mathbf{v} .
\end{aligned}
$$

Evidentemente, ao olhar para as expressões anteriores não é óbvio que as matrizes $D, E$ e vetores $\mathbf{w}^{T}, \mathbf{v}$ existam. Veremos, no entanto, que é possível

${ }^{2}$ Falaremos sobre isso na Seção 5.3. 
escolher essas matrizes e vetores de modo que $f_{N-1}(\eta(1), \cdots, \eta(N-1))$ dada em (5.3) corresponda, de fato, ao peso da configuração $(\eta(1), \cdots, \eta(N-1))$ com respeito ao estado estacionário. Mais adiante, mostraremos quais são as condições que essas matrizes precisam satisfazer para cada tipo de modelo que trataremos nesta dissertação.

Antes de apresentarmos propriedades e formas explícitas para essas matrizes e vetores envolvidos, vamos discutir as vantagens dessa abordagem. Fixemos $\mu$ uma medida de probabilidade inicial qualquer em $\{0,1\}^{\Sigma_{N}}$. De fato, se definirmos a matriz $C=D+E$, a densidade de partículas $\langle\eta(x)\rangle_{\mu} \mathrm{com}$ respeito ao estado estacionário definida por

$$
\langle\eta(x)\rangle_{\mu}=\frac{\sum_{\eta(1) \in\{1,0\}} \cdots \sum_{\eta(N-1) \in\{1,0\}} \eta(x) f(\eta(1), \cdots, \eta(N-1))}{Z_{N-1}},
$$

pode ser calculada através da seguinte expressão

$$
\begin{aligned}
\langle\eta(x)\rangle_{\mu} & =\sum_{\eta(1) \in\{1,0\}} \cdots \sum_{\eta(x-1), \eta(x+1) \in\{0,1\}} \cdots \sum_{\eta(N-1) \in\{1,0\}} \frac{\mathbf{w}^{T} \prod_{j=1}^{x-1} X_{\eta(j)} D \prod_{j=x+1}^{N-1} X_{\eta(j)} \mathbf{v}}{Z_{N-1}} \\
& =\frac{\mathbf{w}^{T} C^{x-1} D C^{N-1-x} \mathbf{v}}{\mathbf{w}^{T} C^{N-1} \mathbf{v}} .
\end{aligned}
$$

Similarmente, quando $x<y$, a esperança entre as variáveis aleatórias $\eta(x)$ e $\eta(y)$ com respeito ao estado estacionário será dada por

$$
\begin{aligned}
\langle\eta(x) \eta(y)\rangle_{\mu} & =\frac{\sum_{\eta(1) \in\{0,1\}} \cdots \sum_{\eta(N-1) \in\{0,1\}} \eta(x) \eta(y) f(\eta(1), \cdots, \eta(N-1))}{Z_{N-1}} \\
& =\frac{\mathbf{w}^{T} C^{x-1} D C^{y-x-1} D C^{N-1-y} \mathbf{v}}{\mathbf{w}^{T} C^{N-1} \mathbf{v}}
\end{aligned}
$$

Também podemos estudar a correlação entre dois sítios, por exemplo, utilizando (5.9) e (5.10), a correlação entre $\eta(x)$ e $\eta(y)$ com respeito ao estado estacionário para $1 \leq x<y \leq N-1$ é dada por

$$
\begin{aligned}
\langle\eta(x) ; \eta(y)\rangle_{\mu} & \equiv\langle\eta(x) \eta(y)\rangle_{\mu}-\langle\eta(x)\rangle_{\mu}\langle\eta(y)\rangle_{\mu} \\
& =\frac{\mathbf{w}^{T} C^{x-1} D C^{y-x-1} D C^{N-1-y} \mathbf{v}}{\mathbf{w}^{T} C^{N-1} \mathbf{v}} \\
& -\frac{\mathbf{w}^{T} C^{x-1} D C^{N-1-x} \mathbf{v}}{\mathbf{w}^{T} C^{N-1} \mathbf{v}} \cdot \frac{\mathbf{w}^{T} C^{y-1} D C^{N-1-y} \mathbf{v}}{\mathbf{w}^{T} C^{N-1} \mathbf{v}} .
\end{aligned}
$$

Além disso, a corrente entre os sítios $x$ e $x+1$ no bulk do ASEP em contato com reservatórios, é dada por

$$
j_{x, x+1}(\eta)=p \eta(x)(1-\eta(x+1))-q \eta(x+1)(1-\eta(x))
$$


Assim, a corrente média entre os sítios $x$ e $x+1$ com respeito ao estado estacionário no bulk do ASEP em contato com reservatórios, é dada por

$$
\left\langle j_{x, x+1}(\eta)\right\rangle_{\mu}=\frac{\mathbf{w}^{T} C^{x-1}(p D E-q E D) C^{N-2-x} \mathbf{v}}{\mathbf{w}^{T} C^{N-1} \mathbf{v}} .
$$

Portanto, se tivermos formas convenientes para as matrizes $D, E$ e para os vetores $\mathbf{w}^{T}, \mathbf{v}$ tal que quaisquer potências de $C$ tenham expressões simples, então as equações (5.9), (5.10), (5.11) e (5.13) serão calculadas facilmente.

Teorema 5.2. Considere o ASEP em $\{1, \cdots, N-1\}$ com taxas de fronteira $\alpha, \beta \in(0,1)$ e taxas $p, q$ no bulk. Suponha que as matrizes $D, E$ e os vetores $\boldsymbol{w}^{T}, \boldsymbol{v}$ satisfazem

$$
\begin{aligned}
p D E-q E D & =D+E \\
\boldsymbol{w}^{T}[\alpha p E-(1-\alpha) q D] & =\boldsymbol{w}^{T} \\
{[(1-\beta) p D-\beta q E] \boldsymbol{v} } & =\boldsymbol{v} .
\end{aligned}
$$

Essas relações são chamadas de álgebra de matrizes. Se para toda configuração $\eta \in\{0,1\}^{\Sigma_{N}}$ a função (5.3) estiver bem definida em $\mathbb{R}^{+}$e a normalização

$$
Z_{N-1}=\sum_{\eta \in \Omega_{N}} f_{N-1}(\eta) \neq 0,
$$

então a medida estacionária do ASEP será dada por

$$
\mu_{s s}(\eta)=\frac{f_{N-1}(\eta)}{Z_{N-1}}
$$

Demonstração. Seja $W(\eta, \xi)$ a taxa na qual o ASEP muda de uma configuração $\eta$ para uma configuração $\xi$ :

$$
W\left(\eta, \eta^{x, x+1}\right)= \begin{cases}p, & \text { se } \eta(x)=1, \eta(x+1)=0 \\ q, & \text { se } \eta(x)=0, \eta(x+1)=1\end{cases}
$$

para $1 \leq x \leq N-2$,

$$
\begin{gathered}
W\left(\eta, \eta^{1}\right)= \begin{cases}\alpha p, & \text { se } \eta(1)=0, \\
(1-\alpha) q, & \text { se } \eta(1)=1,\end{cases} \\
W\left(\eta, \eta^{N-1}\right)= \begin{cases}\beta q, & \text { se } \eta(N-1)=0, \\
(1-\beta) p, & \text { se } \eta(N-1)=1,\end{cases}
\end{gathered}
$$

e $W(\eta, \xi)=0$ caso contrário. Como $\left\{\eta_{t}\right\}_{t \geq 0}$ é uma cadeia de Markov irredutível com espaço de estados finito, ela possui uma única medida estacionária $\mu_{s s}$, 
dada pela solução estacionária da master equation

$$
\frac{d}{d t} \mu_{s s}(\eta)=0=\sum_{\xi \neq \eta}\left(\mu_{s s}(\xi) W(\xi, \eta)-\mu_{s s}(\eta) W(\eta, \xi)\right) \text { para todo } \eta \in \Omega_{N}
$$

Portanto, para mostrar que (5.16) é medida estacionária, é suficiente mostrar que $f_{N-1}$ dada em (5.3) satisfaz (5.17), e então pode ser automaticamente normalizada. No nosso caso, os termos individuais (não normalizados) da soma (5.17) para o bulk são da forma

$$
f_{N-1}\left(\eta^{x, x+1}\right) W\left(\eta^{x, x+1}, \eta\right)-f_{N-1}(\eta) W\left(\eta, \eta^{x, x+1}\right),
$$

similarmente para as fronteiras temos

$$
\begin{aligned}
& f_{N-1}\left(\eta^{1}\right) W\left(\eta^{1}, \eta\right)-f_{N-1}(\eta) W\left(\eta, \eta^{1}\right) \text { e } \\
& f_{N-1}\left(\eta^{N-1}\right) W\left(\eta^{N-1}, \eta\right)-f_{N-1}(\eta) W\left(\eta, \eta^{N-1}\right) .
\end{aligned}
$$

Esses termos podem ser simplificados utilizando a álgebra de matrizes (5.14). De fato, utilizando (5.14a), no caso em que $\eta(x)=1$ e $\eta(x+1)=0$, temos para o bulk

$$
\begin{aligned}
& f_{N-1}(\cdots, \eta(x-1), 0,1, \eta(x+2) \cdots) q-f_{N-1}(\cdots, \eta(x-1), 1,0, \eta(x+2) \cdots) p \\
= & \mathbf{w}^{T} \cdots X_{\eta(x-1)} \underbrace{[q E D-p D E]}_{=-(D+E)} X_{\eta(x+2)} \cdots \mathbf{v} \\
= & -\mathbf{w}^{T} \cdots X_{\eta(x-1)}[D] X_{\eta(x+2)} \cdots \mathbf{v}-\mathbf{w}^{T} \cdots X_{\eta(x-1)}[E] X_{\eta(x+2)} \cdots \mathbf{v} \\
= & -f_{N-2}(\cdots, \eta(x-1), 1, \eta(x+2) \cdots)-f_{N-2}(\cdots, \eta(x-1), 0, \eta(x+2), \cdots) .
\end{aligned}
$$

De modo análogo ao anterior, caso $\eta(x)=0$ e $\eta(x+1)=1$, temos

$$
\begin{aligned}
& f_{N-1}(\cdots, \eta(x-1), 1,0, \eta(x+2), \cdots) p-f_{N-1}(\cdots, \eta(x-1), 0,1, \eta(x+2), \cdots) q \\
& =f_{N-2}(\cdots, \eta(x-1), 1, \eta(x+2), \cdots)+f_{N-2}(\cdots, \eta(x-1), 0, \eta(x+2), \cdots) .
\end{aligned}
$$

Dessa forma, podemos escrever os termos individuais de (5.18) para o bulk como

$$
\begin{aligned}
f_{N-1}\left(\eta^{x, x+1}\right) & W\left(\eta^{x, x+1}, \eta\right)-f_{N-1}(\eta) W\left(\eta, \eta^{x, x+1}\right) \\
& =(1-2 \eta(x)) f_{N-2}(\cdots, \eta(x-1), \eta(x), \eta(x+2), \cdots) \\
& -(1-2 \eta(x+1)) f_{N-2}(\cdots, \eta(x-1), \eta(x+1), \eta(x+2), \cdots) .
\end{aligned}
$$


Para a fronteira esquerda, caso $\eta(1)=1$, temos

$$
\begin{aligned}
\alpha p f_{N-1}(0, \cdots)-(1-\alpha) q f_{N-1}(1, \cdots) & =\mathbf{w}^{T}[\alpha p E] \cdots \mathbf{v}-\mathbf{w}^{T}[(1-\alpha) q D] \cdots \mathbf{v} \\
& =\underbrace{\mathbf{w}^{T}[\alpha p E-(1-\alpha) q D]}_{=\mathbf{w}^{T}} \cdots \mathbf{v} \\
& =\mathbf{w}^{T} \underbrace{\cdots}_{\text {N-2 termos }} \mathbf{v} \\
& =f_{N-2}(\eta(2), \cdots),
\end{aligned}
$$

e caso $\eta(1)=0$

$$
\begin{aligned}
(1-\alpha) q f_{N-1}(1, \cdots)-\alpha p f_{N-1}(0, \cdots) & =\mathbf{w}^{T}[(1-\alpha) q D-\alpha p E] \cdots \mathbf{v} \\
& =-\underbrace{\mathbf{w}^{T}[\alpha p E-(1-\alpha) q D]}_{=\mathbf{w}^{T}} \cdots \mathbf{v} \\
& =-\mathbf{w}^{T} \underbrace{\cdots}_{\mathrm{N}-2 \text { termos }} \mathbf{v} \\
& =-f_{N-2}(\eta(2), \cdots) .
\end{aligned}
$$

Assim, podemos representar os termos da fronteira esquerda por

$$
f_{N-1}\left(\eta^{1}\right) W\left(\eta^{1}, \eta\right)-f_{N-1}(\eta) W\left(\eta, \eta^{1}\right)=-(1-2 \eta(1)) f_{N-2}(\eta(2), \cdots) .
$$

De modo análogo ao realizado anteriormente, podemos representar os termos da fronteira direita por

$$
\begin{aligned}
& f_{N-1}\left(\eta^{N-1}\right) W\left(\eta^{N-1}, \eta\right)-f_{N-1}(\eta) W\left(\eta, \eta^{N-1}\right) \\
& =(1-2 \eta(N-1)) f_{N-2}(\cdots, \eta(N-2)) .
\end{aligned}
$$

A soma sobre todos os $x \in \Sigma_{N}$ em (5.18) mais os termos vindos de (5.22) e (5.23) corresponde ao lado direito de (5.17), e como a soma em (5.18) é telescópica, sobram-se dois termos dessa soma, os quais se cancelam com os termos vindos das equações (5.22) e (5.23), mostrando assim que $\mu_{s s}$ é medida estacionária para o ASEP, como queríamos.

Visto que em um primeiro contato a origem da álgebra de matrizes pode parecer um tanto quanto estranha para o leitor, nossa ideia agora é apresentar uma maneira de obter essa álgebra para um sistema de partículas. Inicialmente, o sistema de partículas de interesse será o ASEP em contato com reservatórios. Com efeito, a maneira mais natural de obtermos a álgebra de matrizes para esse sistema, é utilizando o fato de que a corrente media nas fronteiras e no bulk com respeito ao estado estacionário coincidem, e isso segue da observação abaixo. 
Observação 5.3. Se $\mu_{s s}$ é uma medida estacionária em $\{0,1\}^{\Sigma_{N}}$, então $\int L_{N} f(\eta) d \mu_{s s}=0$ para qualquer $f:\{0,1\}^{\Sigma_{N}} \rightarrow \mathbb{R}$, veja [18] para mais detalhes. Assim, tomando $f_{x}:\{0,1\}^{\Sigma_{N}} \rightarrow \mathbb{R}$ onde $f_{x}(\eta)=\eta(x)$, temos que

$$
\int L_{N} f_{x}(\eta) d \mu_{s s}=\int\left(j_{x-1, x}(\eta)-j_{x, x+1}(\eta)\right) d \mu_{s s}=0
$$

para $x \in \Sigma_{N}$. Logo, para $x \in \Sigma_{N}$,

$$
\int j_{x-1, x}(\eta) d \mu_{s s}=\int j_{x, x+1}(\eta) d \mu_{s s}
$$

isto é, no estado estacionário a corrente média no bulk e nas fronteiras é constante.

Por exemplo, por (5.13), a corrente microscópica média com respeito à medida estacionária $\mu_{s s}$ no bulk e nas fronteiras para o ASEP é dada por

$$
\begin{aligned}
\underbrace{\frac{\mathbf{w}^{T}[\alpha p E-(1-\alpha) q D] C^{N-2} \mathbf{v}}{Z_{N-1}}}_{\text {fronteira esquerda }} & =\underbrace{\frac{\mathbf{w}^{T} C^{x-1}(p D E-q E D) C^{(N-1)-(x+1)} \mathbf{v}}{Z_{N-1}}}_{\text {bulk }} \\
& =\underbrace{\frac{\mathbf{w}^{T} C^{N-2}[(1-\beta) p D-\beta q E-] \mathbf{v}}{Z_{N-1}}}_{\text {fronteira direita }} .
\end{aligned}
$$

Assim, podemos verificar que as condições que as matrizes precisam satisfazer para garantir que a corrente em (5.24) seja constante, são exatamente as dadas em (5.14). Além disso, motivados pela descrição apresentada acima, no Capítulo 6, seremos capazes de obter essa álgebra de matrizes para o PMM em contato com reservatórios, e para o SSEP em contato com reservatórios lentos.

Neste ponto da dissertação restam ainda algumas perguntas a serem respondidas no que diz respeito às propriedades que as matrizes $D, E$ e os vetores $\mathbf{w}^{T}, \mathbf{v}$ devem satisfazer. Qual seria a dimensão dessas matrizes? Será que elas comutam? Essas são algumas perguntas que serão respondidas na próxima Seção.

\section{3}

\section{Propriedades e exemplos das matrizes}

Para que o Teorema 5.2 seja útil, devemos ser capazes de encontrar matrizes e vetores que satisfaçam (5.14). O primeiro passo que daremos nesta direção é saber se as matrizes $D$ e $E$ podem comutar e/ou serem de dimensão finita. 
Proposição 5.4. Suponha que D, E, $\boldsymbol{w}^{T}$ e $\boldsymbol{v}$ satisfazem (5.14) e $f_{N-1}$ definida em (5.3) é estritamente positiva.

(a) Se D e E comutam, então

(i) $\alpha=\beta$, ou

(ii) $p=1$ e $\alpha=0$, ou $p=1$ e $\beta=1$, ou

(iii) $p=0$ e $\alpha=1$, ou $p=0$ e $\beta=0$.

No caso que (i), a única medida estacionária é $\nu_{\alpha}^{N}$ (Bernoulli produto, que foi definida na Definição 2.5), enquanto nos casos (ii) e (iii), as medidas estacionários concentram-se em configurações da forma

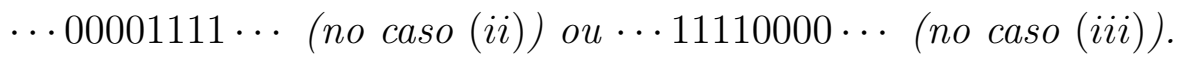

(b) Se $p=1$ e $D$ e E não comutam, então elas são necessariamente de dimensão infinita.

Demonstração. Vide [16] página 264.

Observação 5.5. De agora em diante vamos excluir o caso trivial (a) da Proposição 5.4. Portanto, as matrizes $D$ e E (se elas existirem) não necessariamente comutarão e serão geralmente de dimensão infinita.

As matrizes mais simples que podemos pensar para $D$ e $E$ são as matrizes diagonais, porém, assim sendo, elas comutam. Dessa forma, tendo em vista a Observação 5.5, não será útil para nós que essas matrizes sejam diagonais. O próximo caso mais simples é o das matrizes bi-diagonais, que pode ser visto em [16] página 266. 


\section{6}

\section{Utilizando a matriz ansatz}

O objetivo deste capítulo é obtermos informações sobre o estado estacionário de um sistema de partículas de interesse. Assim, utilizaremos o método da matriz ansatz para obtermos essas informações para os seguintes sistemas de partículas: para o SSEP em contato com reservatórios, para o PMM em contato com reservatórios, e por fim, para o SSEP em contato com reservatórios lentos (todos esses processos foram definidos no Capítulo 2). Os resultados obtidos no capítulo anterior utilizando o método da matriz ansatz nos dão uma maneira simples para escrever a densidade de partículas, a corrente microscópica média, e as funções de correlação em ordens superiores para o processo de interesse. Mas estamos interessados em saber qual o real valor dessas expressões, isto é, dado um resultado na forma matricial, como obter o seu real valor? Para isso, precisaremos fazer o processo contrário ao que foi feito no Capítulo 5, e o primeiro passo neste sentido é encontrar uma expressão para a normalização (5.8).

Antes de apresentarmos a expressão para $Z_{N-1}$, vamos introduzir algumas notações. Considere o SSEP em contato com reservatórios com as seguintes taxas nas fronteiras: as partículas entram para o sítio 1 com taxa $\alpha$ (respectivamente para o sítio $N-1 \mathrm{com}$ taxa $\beta$ ), e saem do sítio $1 \mathrm{com}$ taxa $\gamma$ (respectivamente do sítio $N-1$ com taxa $\delta$ ), como pode ser visto na figura abaixo.

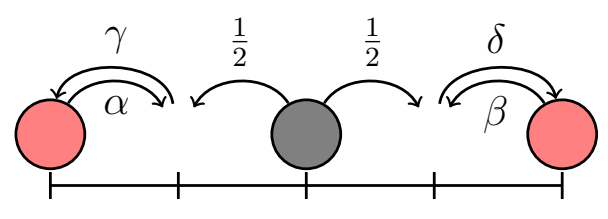

Figura 6.1: SSEP em contato com reservatórios.

De [7] a expressão para a normalização $Z_{N-1}$ é dada por

$$
Z_{N-1}=\frac{1}{\left(\rho_{a}-\rho_{b}\right)^{N-1}} \frac{\Gamma\left(N-1+\frac{1}{\alpha+\gamma}+\frac{1}{\beta+\delta}\right)}{\Gamma\left(\frac{1}{\alpha+\gamma}+\frac{1}{\beta+\delta}\right)},
$$

onde $\rho_{a}=\frac{\alpha}{\alpha+\gamma}, \rho_{b}=\frac{\delta}{\beta+\delta}, \Gamma(z)$ é a função Gama usual dada por $\Gamma(z+1)=z$ ! 
para $z$ sendo um número inteiro positivo, e $\Gamma(z)=\int_{0}^{\infty} x^{z-1} e^{-x} d x$ para qualquer $z \in \mathbb{C} \operatorname{com} \operatorname{Re}(z)>0$.

\section{1}

\section{A matriz ansatz para SSEP em contato com reservatórios}

Utilizando os resultados apresentados no capítulo anterior para $p=q=$ 1, observamos que, após algumas manipulações algébricas, as equações (5.14b) e (5.14c) podem ser escritas como

$$
\mathbf{w}^{T} D=\mathbf{w}^{T}(\alpha C-1), \quad D \mathbf{v}=(\beta C+1) \mathbf{v} .
$$

Outro fato que devemos observar manipulando essas equações é que

$$
C(D+I)=(D+E)(D+I)=D D+D+E D+E
$$

e como por (5.14a) $E D=D E-C$, segue que $C(D+I)=D D+D E=D C$. De modo análogo obtemos que $C D=(D-I) C$. Logo, por (5.14a) temos

$$
C(D+I)=D C \quad \text { e } \quad C D=(D-I) C .
$$

Utilizando (6.1), a normalização $Z_{N-1}$ para o SSEP em contato com reservatórios é dada por

$$
Z_{N-1}=\frac{1}{(\alpha-\beta)^{N-1}} \frac{\Gamma(N+1)}{\Gamma(2)}=\frac{N !}{(\alpha-\beta)^{N-1}} .
$$

Sendo $\mu_{s s}$ uma medida estacionária, e utilizando (5.14a) com $p=q=1$, (6.2) e (6.3), podemos escrever a densidade de partículas em um sítio $x \in \Sigma_{N}$ como

$$
\begin{aligned}
\langle\eta(x)\rangle_{\mu_{s s}}^{N-1} & =\frac{\mathbf{w}^{T} \overbrace{C^{x-1} D} C^{N-1-x} \mathbf{v}}{Z_{N-1}} \\
& =\frac{\mathbf{w}^{T} C^{x-2}(D-I) C^{N-x} \mathbf{v}}{Z_{N-1}} \\
& =\frac{\mathbf{w}^{T} \overbrace{C^{x-2} D} C^{N-x} \mathbf{v}}{Z_{N-1}}-\frac{\mathbf{w}^{T} C^{N-2} \mathbf{v}}{Z_{N-1}} \\
& =\frac{\mathbf{w}^{T} C^{x-3}(D-I) C^{N-x+1} \mathbf{v}-\mathbf{w}^{T} C^{N-2} \mathbf{v}}{Z_{N-1}} \\
& =\cdots=\frac{\mathbf{w}^{T} D C^{(N-1-x)+(x-1)} \mathbf{v}}{Z_{N-1}}-(x-1) \frac{\mathbf{w}^{T} C^{N-2} \mathbf{v}}{Z_{N-1}} \\
& =\frac{\mathbf{w}^{T} D C^{N-2} \mathbf{v}}{Z_{N-1}-(x-1) \frac{\mathbf{w}^{T} C^{N-2} \mathbf{v}}{Z_{N-1}}} \\
& =\frac{\alpha \mathbf{w}^{T} C^{N-1} \mathbf{v}-x \mathbf{w}^{T} C^{N-2} \mathbf{v}}{Z_{N-1}}
\end{aligned}
$$




$$
\begin{aligned}
& =\alpha-\frac{x(\alpha-\beta)^{N-1}}{N !} \frac{(N-1) !}{(\alpha-\beta)^{N-2}} \\
& =\frac{\alpha(N-x)+x \beta}{N},
\end{aligned}
$$

que está de acordo com o resultado obtido em (4.8). Já a esperança nos sítios $x, y \in \Sigma_{N}$ para $x<y$ com respeito à medida estacionária $\mu_{s s}$ é dada por

$$
\begin{aligned}
& \langle\eta(x) \eta(y)\rangle_{\mu_{s s}}^{N-1}=\frac{\mathbf{w}^{T} \overbrace{C^{x-1} D} C^{y-x-1} D C^{N-1-y} \mathbf{v}}{Z_{N-1}} \\
& =\frac{\mathbf{w}^{T} C^{x-2}(D-I) C^{y-x} D C^{N-1-y} \mathbf{v}}{Z_{N-1}} \\
& =\frac{\mathbf{w}^{T} \overbrace{C^{x-2} D} C^{y-x} D C^{N-1-y} \mathbf{v}-\mathbf{w}^{T} C^{y-2} D C^{N-1-y} \mathbf{v}}{Z_{N-1}} \\
& =\frac{\mathbf{w}^{T} C^{x-3} D C^{y-x+1} D C^{N-1-y} \mathbf{v}}{Z_{N-1}}-\frac{2 \mathbf{w}^{T} C^{y-2} D C^{N-1-y} \mathbf{v}}{Z_{N-1}} \\
& =\cdots=\frac{\mathbf{w}^{T} C^{(x-1)-(x-1)} D C^{(y-x-1)+(x-1)} D C^{N-1-y} \mathbf{v}}{Z_{N-1}} \\
& -\frac{(x-1) \mathbf{w}^{T} C^{y-2} D C^{N-1-y}}{Z_{N-1}} \\
& =\frac{\overbrace{\mathbf{w}^{T} D} C^{y-2} D C^{N-1-y} \mathbf{v}-(x-1) \mathbf{w}^{T} C^{y-2} D C^{N-1-y}}{Z_{N-1}} \\
& =\frac{\mathbf{w}^{T}(\alpha C-1) C^{y-2} D C^{N-1-y} \mathbf{v}-(x-1) \mathbf{w}^{T} C^{y-2} D C^{N-1-y} \mathbf{v}}{Z_{N-1}} \\
& =\alpha \frac{\mathbf{w}^{T} C^{y-1} D C^{N-1-y} \mathbf{v}}{Z_{N-1}}-x \frac{\mathbf{w}^{T} \overbrace{C^{y-2} D} C^{N-1-y} \mathbf{v}}{Z_{N-1}} \\
& =\alpha\langle\eta(y)\rangle_{\mu_{s s}}^{N-1}-x \alpha \frac{\mathbf{w}^{T} C^{N-2} \mathbf{v}}{Z_{N-1}}+x(y-1) \frac{\mathbf{w}^{T} C^{N-3} \mathbf{v}}{Z_{N-1}} \\
& =\alpha\langle\eta(y)\rangle_{\mu_{s s}}^{N-1}-x \alpha \frac{Z_{N-2}}{Z_{N-1}}+x(y-1) \frac{Z_{N-3}}{Z_{N-1}} \\
& =\alpha\left[\frac{\alpha(N-y)+y \beta}{N}\right]-\alpha x\left[\frac{(N-1) !}{(\alpha-\beta)^{N-2}} \frac{(\alpha-\beta)^{N-1}}{N !}\right] \\
& +x(y-1)\left[\frac{(N-2) !}{(\alpha-\beta)^{N-3}} \frac{(\alpha-\beta)^{N-1}}{N !}\right] \\
& =\frac{\alpha^{2}(N-y)+\alpha \beta y}{N}-\alpha x \frac{(\alpha-\beta)}{N}+x(y-1) \frac{(\alpha-\beta)^{2}}{N(N-1)} \\
& =\frac{\alpha^{2}(N-y)+\alpha \beta y-x \alpha(\alpha-\beta)}{N}+x(y-1) \frac{(\alpha-\beta)^{2}}{N(N-1)} \\
& =\frac{\alpha^{2}[(N-y)(N-1)+x(y-1)-x(N-1)]}{N(N-1)} \\
& +\frac{\alpha \beta[y(N-1)+x(N-1)-2 x(y-1)]+\beta^{2} x(y-1)}{N(N-1)} .
\end{aligned}
$$


Portanto

$$
\begin{aligned}
\langle\eta(x) \eta(y)\rangle_{\mu_{s s}}^{N-1} & =\frac{\alpha^{2}(N-y)(N-x-1)+\alpha \beta((N-1)(x+y)-2 x(y-1))}{N(N-1)} \\
& +\frac{\beta^{2} x(y-1)}{N(N-1)} .
\end{aligned}
$$

Lembremos que a corrente microscópica entre os sítios $x$ e $x+1$ para o SSEP em contato com reservatórios, calculada no Capítulo 4, Seção 4.1, é dada por

$$
j_{x, x+1}(\eta)=\eta(x)(1-\eta(x+1))-\eta(x+1)(1-\eta(x)) .
$$

Assim, fazendo o cálculo da corrente microscópica média entre os sítios $x$ e $x+1$ temos

$$
\begin{aligned}
\left\langle j_{x, x+1}(\eta)\right\rangle_{\mu_{s s}} & =\frac{\mathbf{w}^{T} C^{x-1}(D E-E D) C^{N-x-2} \mathbf{v}}{\mathbf{w}^{T} C^{N-1} \mathbf{v}} \\
& =\frac{\mathbf{w}^{T} C^{N-2} \mathbf{v}}{\mathbf{w}^{T} C^{N-1} \mathbf{v}} \\
& =\frac{Z_{N-2}}{Z_{N-1}} \\
& =\frac{(N-1) !}{(\alpha-\beta)^{N-2}} \frac{(\alpha-\beta)^{N-1}}{N !} \\
& =\frac{\alpha-\beta}{N},
\end{aligned}
$$

que também está de acordo com a corrente microscópica média calculada em (4.11). Queremos calcular agora a correlação entre $\eta(x)$ e $\eta(y)$ com respeito à medida estacionária. Para isso, consideremos a seguinte definição.

Definição 6.1. A correlação (ou covariância) entre duas variáveis aleatórias $X$ e $Y$ é igual à esperança do produto menos o produto das esperanças, isto é:

$$
\operatorname{Cov}(X, Y)=\mathbb{E}[X Y]-\mathbb{E}[X] \mathbb{E}[Y]
$$

sempre que as esperanças envolvidas estejam bem definidas.

Denotaremos a correlação entre $\eta(x)$ e $\eta(y)$ sob a medida estacionária $\mu_{s s}$ como

$$
\langle\eta(x) ; \eta(y)\rangle_{\mu_{s s}}^{N-1}=\langle\eta(x) \eta(y)\rangle_{\mu_{s s}}^{N-1}-\langle\eta(x)\rangle_{\mu_{s s}}^{N-1}\langle\eta(y)\rangle_{\mu_{s s}}^{N-1} .
$$

Portanto, como consequência de (6.5) e (6.6), a correlação entre $\eta(x)$ e $\eta(y)$ sob a medida estacionária $\mu_{s s}$ é dada por 


$$
\begin{aligned}
\langle\eta(x) ; \eta(y)\rangle_{\mu_{s s}}^{N-1} & =\frac{\alpha^{2}(N-y)(N-x-1)+\alpha \beta((N-1)(x+y)-2 x(y-1))}{N(N-1)} \\
& +\frac{\beta^{2} x(y-1)}{N(N-1)}-\left[\frac{\alpha(N-x)+x \beta}{N}\right]\left[\frac{\alpha(N-y)+y \beta}{N}\right] \\
& =\alpha^{2} \frac{[(N-y)(N-x-1) N-(N-x)(N-y)(N-1)]}{N^{2}(N-1)} \\
& +\alpha \beta \frac{[((N-1)(x+y)-2 x(y-1)) N-(N x+N y-2 x y)(N-1)]}{N^{2}(N-1)} \\
& +\beta^{2} \frac{[x(y-1) N-x y(N-1)]}{N^{2}(N-1)} \\
& =-\frac{x(N-y)}{N^{2}(N-1)}(\alpha-\beta)^{2} .
\end{aligned}
$$

\section{2}

\section{A matriz ansatz para o PMM em contato com reservatórios}

Nesta Seção pretendemos repetir os cálculos feitos no Capítulo 4 para encontrar a álgebra de matrizes para o PMM em contato com reservatórios. Podemos notar que para o PMM a abordagem usada na equação (4.4) não é bem sucedida. De fato, a evolução temporal da densidade de partículas em cada sítio $x \in \Sigma_{N}$ com respeito a uma medida $\mu$ qualquer, é dada por

$$
\begin{aligned}
\frac{d}{d t}\left\langle\eta_{t}(1)\right\rangle_{\mu}= & \left\langle\alpha-\eta_{t}(1)-\left(\eta_{t}(1)-\eta_{t}(2)\right)\left(\alpha+\eta_{t}(3)\right)\right\rangle_{\mu} \\
= & \alpha-(1+\alpha)\left\langle\eta_{t}(1)\right\rangle_{\mu}+\alpha\left\langle\eta_{t}(2)\right\rangle_{\mu} \\
- & \left\langle\eta_{t}(1) \eta_{t}(3)\right\rangle_{\mu}+\left\langle\eta_{t}(2) \eta_{t}(3)\right\rangle_{\mu}, \\
\frac{d}{d t}\left\langle\eta_{t}(x)\right\rangle_{\mu}= & \left\langle\eta_{t}(x-2) \eta_{t}(x-1)\right\rangle_{\mu}-\left\langle\eta_{t}(x-2) \eta_{t}(x)\right\rangle_{\mu} \\
- & \left\langle\eta_{t}(x-1) \eta_{t}(x)\right\rangle_{\mu}+2\left\langle\eta_{t}(x-1) \eta_{t}(x+1)\right\rangle_{\mu} \\
- & \left\langle\eta_{t}(x) \eta_{t}(x+1)\right\rangle_{\mu}-\left\langle\eta_{t}(x) \eta_{t}(x+2)\right\rangle_{\mu} \\
+ & \left\langle\eta_{t}(x+1) \eta_{t}(x+2)\right\rangle_{\mu} \\
& \operatorname{para} x \in\{2, \cdots, N-2\} \\
\frac{d}{d t}\left\langle\eta_{t}(N-1)\right\rangle_{\mu}= & \left\langle\eta_{t}(N-3) \eta_{t}(N-2)\right\rangle_{\mu}-\left\langle\eta_{t}(N-3) \eta_{t}(N-1)\right\rangle_{\mu} \\
+ & \beta\left\langle\eta_{t}(N-2)\right\rangle_{\mu}-(\beta+1)\left\langle\eta_{t}(N-1)\right\rangle_{\mu}+\beta
\end{aligned}
$$

Em princípio podemos calcular a evolução temporal da densidade de partículas. Entretanto, o problema é que o cálculo da densidade $\left\langle\eta_{t}(x)\right\rangle_{\mu}$ requer o conhecimento da esperança entre dois sítios quaisquer $\left\langle\eta_{t}(x) \eta_{t}(y)\right\rangle_{\mu}$, que por sua vez requer o conhecimento da esperança entre três sítios quaisquer e assim 
sucessivamente, o que torna o problema cada vez mais difícil.

Dada uma configuração $\eta \in\{0,1\}^{\Sigma_{N}}$ a corrente microscópica média entre os sítios $x$ e $x+1$ no bulk do PMM em contato com reservatórios com respeito a uma medida $\mu$ qualquer é dada por

$$
\begin{aligned}
\left\langle j_{x, x+1}(\eta)\right\rangle_{\mu} & =\langle(\eta(x)-\eta(x+1))(\eta(x-1)+\eta(x+2))\rangle_{\mu} \\
& =\langle\eta(x-1)(\eta(x)(1-\eta(x+1))-\eta(x+1)(1-\eta(x))) \\
& +(\eta(x)(1-\eta(x+1))-\eta(x+1)(1-\eta(x))) \eta(x+2)\rangle_{\mu} .
\end{aligned}
$$

A segunda igualdade de (6.10) representa uma forma de escrever a corrente que será útil abaixo. Dada uma medida estacionária $\mu_{s s}$ podemos escrever (6.10) na forma matricial como

$$
\begin{aligned}
\left\langle j_{x, x+1}(\eta)\right\rangle_{\mu_{s s}} & =\frac{\mathbf{w}^{T} C^{x-2} D[D E-E D] C^{(N-1)-(x+1)} \mathbf{v}}{Z_{N-1}} \\
& +\frac{\mathbf{w}^{T} C^{x-1}[D E-E D] D C^{(N-1)-(x+2)} \mathbf{v}}{Z_{N-1}} \\
& =\frac{\mathbf{w}^{T} C^{x-2}[D D E C-D E D C+C D E D-C E D D] C^{(N-1)-(x+2)}}{Z_{N-1}} \\
& =\frac{\mathbf{w}^{T} C^{x-2}[(D D E-D E D) C+C(D E D-E D D)] C^{(N-1)-(x+2)} \mathbf{v}}{Z_{N-1}} .
\end{aligned}
$$

Nas fronteiras temos

$$
\begin{aligned}
\left\langle j_{0,1}(\eta)\right\rangle_{\mu_{s s}} & =\frac{\mathbf{w}^{T}(\alpha E-(1-\alpha) D) C^{N-2} \mathbf{v}}{Z_{N-1}} \\
\left\langle j_{N-1, N}(\eta)\right\rangle_{\mu_{s s}} & =\frac{\mathbf{w}^{T} C^{N-2}((1-\beta) D-\beta E) \mathbf{v}}{Z_{N-1}} .
\end{aligned}
$$

Lembrando da Observação 5.3, a esperança com respeito ao estado estacionário da corrente no bulk e nas fronteiras devem coincidir. Assim

$$
\begin{aligned}
\left\langle j_{x, x+1}(\eta)\right\rangle_{\mu_{s s}} & =\frac{\mathbf{w}^{T}(\alpha E-(1-\alpha) D) C^{N-2} \mathbf{v}}{Z_{N-1}}=\frac{\mathbf{w}^{T} C^{N-2}((1-\beta) D-\beta E) \mathbf{v}}{Z_{N-1}} \\
& =\frac{\mathbf{w}^{T} C^{x-2}[(D D E-D E D) C+C(D E D-E D D)] C^{(N-1)-(x+2)} \mathbf{v}}{Z_{N-1}} .
\end{aligned}
$$

Para termos a igualdade em (6.13) as matrizes precisam satisfazer as seguintes 
condições

$$
\begin{array}{r}
D D E-D E D=D D+E D=C D \\
D E D-E D D=E E+E D=E C, \\
\mathbf{w}^{T}(\alpha E-(1-\alpha) D)=\mathbf{w}^{T}, \\
((1-\beta) D-\beta E) \mathbf{v}=\mathbf{v} .
\end{array}
$$

Portanto, (6.14) é a álgebra de matrizes para o PMM em contato com reservatórios. As propriedades (6.14c) e (6.14d) são obtidas do mesmo modo que no SSEP em contato com reservatórios. Já as propriedades (6.14a) e (6.14b) são obtidas analisando o produto matricial $C C C$ e perguntando-se quais as propriedades que as expressões $D D E-D E D$ e $D E D-E D D$ devem satisfazer de modo que o numerador de (6.13) seja constante, isto é, dado por $\mathbf{w}^{T} C^{N-2} \mathbf{v}$.

\section{3}

\section{A matriz ansatz para o SSEP em contato com reservatórios lentos}

Nesta seção vamos utilizar o método da matriz ansatz para obtermos informações com respeito ao estado estacionário do processo de exclusão simples simétrico em contato com reservatórios lentos, que definimos no Capítulo 2. Esse processo foi estudado em [1], onde foi apresentada uma expressão para a densidade de partículas, bem como uma cota superior para a função de correlação de segunda ordem, ambas com respeito ao estado estacionário.

Assim, vamos utilizar o método da matriz ansatz para calcular a densidade de partículas com respeito ao estado estacionário, e verificaremos que o valor encontrado está de acordo com o Lema 3.1 de [1]. Depois, obteremos pela primeira vez uma expressão explícita para a função de correlação de segunda ordem. Deste modo, utilizando o método visto nas seções anteriores, e calculando a normalização dada em (6.1) para este processo, segue que a álgebra de matrizes para o processo é dada por

$$
\begin{gathered}
D E-E D=D+E \\
\mathbf{w}^{T}\left[\frac{\alpha}{N^{\theta}} E-\frac{1-\alpha}{N^{\theta}} D\right]=\mathbf{w}^{T},\left[\frac{1-\beta}{N^{\theta}} D-\frac{\beta}{N^{\theta}} E\right] \mathbf{v}=\mathbf{v} .
\end{gathered}
$$

Fazendo algumas manipulações de interesse em (6.15) e (6.16) temos

$$
\mathbf{w}^{T} D=\mathbf{w}^{T}\left[\alpha C-N^{\theta}\right], D \mathbf{v}=\left[N^{\theta}+\beta C\right] \mathbf{v}, \mathbf{w}^{T} E=\mathbf{w}^{T}\left[\frac{N^{\theta}}{\alpha}+\frac{1-\alpha}{\alpha} D\right] .
$$

Utilizando (6.1), a normalização $Z_{N-1}$ do SSEP em contato com reservatórios 
lentos será dada por

$$
Z_{N-1}=\frac{1}{(\alpha-\beta)^{N-1}} \frac{\Gamma\left(2 N^{\theta}+N-1\right)}{\Gamma\left(2 N^{\theta}\right)} .
$$

Assim, a densidade de partículas em um sítio $x$ com respeito a medida estacionária $\mu_{s s}$ é dada por

$$
\begin{aligned}
& \langle\eta(x)\rangle_{\mu_{s s}}^{N-1}=\frac{\mathbf{w}^{T} C^{x-1} \overparen{\overbrace{D C^{N-1-x}}} \mathbf{v}}{Z_{N-1}} \\
& =\frac{\mathbf{w}^{T} C^{x-1} C(D+I) C^{N-2-x} \mathbf{v}}{Z_{N-1}} \\
& =\frac{\mathbf{w}^{T} C^{x} \overbrace{D C^{N-2-x}} \mathbf{v}}{Z_{N-1}}+\frac{\mathbf{w}^{T} C^{N-2} \mathbf{v}}{Z_{N-1}} \\
& =\frac{\mathbf{w} C^{x+1} D C^{N-3-x} \mathbf{v}}{Z_{N-1}}+2 \frac{\mathbf{w}^{T} C^{N-2} \mathbf{v}}{Z_{N-1}} \\
& =\cdots=\frac{\mathbf{w}^{t} C^{(x-1)+N-1-x} D C^{(N-1-x)-(N-1-x)} \mathbf{v}}{Z_{N-1}}+(N-1-x) \frac{Z_{N-2}}{Z_{N-1}} \\
& =\frac{\mathbf{w}^{T} C^{N-2} D \mathbf{v}}{Z_{N-1}}+(N-1-x) \frac{Z_{N-2}}{Z_{N-1}} \\
& =\frac{\mathbf{w}^{T} C^{N-2}\left(N^{\theta}+\beta C\right) \mathbf{v}}{Z_{N-1}}+(N-1-x) \frac{Z_{N-2}}{Z_{N-1}} \\
& =\frac{\beta \mathbf{w}^{T} C^{N-1} \mathbf{v}}{Z_{N-1}}+\left(N-x+N^{\theta}-1\right) \frac{Z_{N-2}}{Z_{N-1}} \\
& =\beta+\left(N-x+N^{\theta}-1\right) \frac{Z_{N-2}}{Z_{N-1}} \\
& =\beta+\left(N-x+N^{\theta}-1\right) \frac{\Gamma\left(2 N^{\theta}+N-2\right)}{(\alpha-\beta)^{N-2} \Gamma\left(2 N^{\theta}\right)} \frac{(\alpha-\beta)^{N-1} \Gamma\left(2 N^{\theta}\right)}{\Gamma\left(2 N^{\theta}+N-1\right)} \\
& =\beta+\left(N-x+N^{\theta}-1\right) \frac{\Gamma\left(2 N^{\theta}+N-2\right)(\alpha-\beta)}{\Gamma\left(2 N^{\theta}+N-2\right)\left(2 N^{\theta}+N-2\right)} .
\end{aligned}
$$

Então

$$
\begin{aligned}
\langle\eta(x)\rangle_{\mu_{s s}}^{N-1} & =\beta+\frac{(\alpha-\beta)\left(N-x+N^{\theta}-1\right)}{2 N^{\theta}+N-2} \\
& =\beta+(N-x) \frac{\alpha-\beta}{2 N^{\theta}+N-2}+\left(N^{\theta}-1\right) \frac{\alpha-\beta}{2 N^{\theta}+N-2}
\end{aligned}
$$

que coincide com o resultado obtido no Lema 3.1 de [1]. Agora vamos calcular a média de partículas nos sítios $x$ e $y$ com respeito à medida estacionária $\mu_{s s}$

$$
\begin{aligned}
\langle\eta(x) \eta(y)\rangle_{\mu_{s s}}^{N-1} & =\frac{\mathbf{w}^{T} C^{x-1} D C^{y-x-1} \overbrace{D C^{N-1-y}} \mathbf{v}}{Z_{N-1}} \\
& =\frac{\mathbf{w}^{T} C^{x-1} D C^{y-x} \overbrace{D C^{N-2-y}} \mathbf{v}}{Z_{N-1}}+\frac{\mathbf{w}^{T} C^{x-1} D C^{N-2-x} \mathbf{v}}{Z_{N-1}}
\end{aligned}
$$




$$
\begin{aligned}
& =\frac{\mathbf{w}^{T} C^{x-1} D C^{y-x+1} D C^{N-3-y} \mathbf{v}}{Z_{N-1}}+2 \frac{\mathbf{w}^{T} C^{x-1} D C^{N-2-x} \mathbf{v}}{Z_{N-1}} \\
& =\cdots=\frac{\mathbf{w}^{T} C^{x-1} D C^{N-2-x} \overbrace{D \mathbf{v}}}{Z_{N-1}}+(N-1-y) \frac{\mathbf{w}^{T} C^{x-1} D C^{N-2-x} \mathbf{v}}{Z_{N-1}} \\
& =\frac{\mathbf{w}^{T} C^{x-1} D C^{N-2-x}\left[N^{\theta}+\beta C\right] \mathbf{v}}{Z_{N-1}}+(N-1-y) \frac{\mathbf{w}^{T} C^{x-1} D C^{N-2-x} \mathbf{v}}{Z_{N-1}} \\
& =\beta \frac{\mathbf{w}^{T} C^{x-1} D C^{N-1-x} \mathbf{v}}{Z_{N-1}}+\left(N-y+N^{\theta}-1\right) \frac{\mathbf{w}^{T} C^{x-1} D C^{N-2-x} \mathbf{v}}{Z_{N-1}} \\
& =\beta\langle\eta(x)\rangle_{\mu_{s s}}^{N-1}+\left(N-y+N^{\theta}-1\right) \frac{(\alpha-\beta)}{\left(2 N^{\theta}+N-2\right)} \frac{\mathbf{w}^{T} C^{x-1} D C^{N-2-x} \mathbf{v}}{Z_{N-2}} \\
& =\beta\langle\eta(x)\rangle_{\mu_{s s}}^{N-1}+\left(N-y+N^{\theta}-1\right) \frac{\alpha-\beta}{2 N^{\theta}+N-2}\langle\eta(x)\rangle_{\mu_{s s}}^{N-2} .
\end{aligned}
$$

Então, usando (6.19), a última igualdade torna-se

$$
\begin{aligned}
\langle\eta(x) \eta(y)\rangle_{\mu_{s s}}^{N-1} & =\beta\left[\beta+\frac{(\alpha-\beta)\left(N-x+N^{\theta}-1\right)}{2 N^{\theta}+N-2}\right] \\
& +\frac{\left(N-y+N^{\theta}-1\right)(\alpha-\beta)}{2 N^{\theta}+N-2}\left[\beta+\frac{(\alpha-\beta)\left(N-x+N^{\theta}-2\right)}{2 N^{\theta}+N-3}\right] \\
& =\beta\left[\frac{\beta\left(x+N^{\theta}-1\right)+\alpha\left(N-x+N^{\theta}-1\right)}{2 N^{\theta}+N-2}\right] \\
& +\frac{\left(N-y+N^{\theta}-1\right)(\alpha-\beta)}{2 N^{\theta}+N-2}\left[\frac{\beta\left(x+N^{\theta}-1\right)+\alpha\left(N-x+N^{\theta}-2\right)}{2 N^{\theta}+N-3}\right] .
\end{aligned}
$$

Com as expressões (6.19) e (6.20), somos capazes de calcular a correlação entre dois sítios quaisquer $x, y \in \Sigma_{N}$ temos

$$
\begin{aligned}
\langle\eta(x) ; \eta(y)\rangle_{\mu_{s s}}^{N-1} & =\langle\eta(x) \eta(y)\rangle_{\mu_{s s}}^{N-1}-\langle\eta(x)\rangle_{\mu_{s s}}^{N-1}\langle\eta(y)\rangle_{\mu_{s s}}^{N-1} \\
& =\left[\left(\beta^{2}\left(x+N^{\theta}-1\right)+\alpha \beta\left(N-x+N^{\theta}-1\right)\right)\left(2 N^{\theta}+N-3\right)\right. \\
& +\left(\alpha \beta\left(x+N^{\theta}-1\right)+\alpha^{2}\left(N-x+N^{\theta}-2\right)-\beta^{2}\left(x+N^{\theta}-1\right)\right. \\
& \left.\left.-\alpha \beta\left(N-x+N^{\theta}-2\right)\right)\left(N-y+N^{\theta}-1\right)\right]\left[\left(2 N^{\theta}+N\right.\right. \\
& \left.-2)\left(2 N^{\theta}+N-3\right)\right]^{-1} \\
& -\left[\beta\left(N^{\theta}+x-1\right)+\alpha\left(N-x+N^{\theta}-1\right)\right]\left[\beta\left(N^{\theta}+y-1\right)\right. \\
& \left.+\alpha\left(N-y+N^{\theta}-1\right)\right]\left[\left(2 N^{\theta}+N-2\right)^{2}\right] \\
& =\left[\beta^{2}\left(x+N^{\theta}-1\right)\left(2 N^{\theta}+N-2\right)\left(2 N^{\theta}+N-3\right)\right. \\
& +\alpha \beta\left(N-x+N^{\theta}-1\right)\left(2 N^{\theta}+N-2\right)\left(2 N^{\theta}+N-3\right)
\end{aligned}
$$




$$
\begin{aligned}
& +\alpha \beta\left(x+N^{\theta}-1\right)\left(N-y+N^{\theta}-1\right)\left(2 N^{\theta}+N-2\right) \\
& +\alpha^{2}\left(N-x+N^{\theta}-2\right)\left(N-y+N^{\theta}-1\right)\left(2 N^{\theta}+N-2\right) \\
& -\beta^{2}\left(x+N^{\theta}-1\right)\left(N-y+N^{\theta}-1\right)\left(2 N^{\theta}+N-2\right) \\
& -\alpha \beta\left(N-x+N^{\theta}-2\right)\left(N-y+N^{\theta}-1\right)\left(2 N^{\theta}+N-2\right) \\
& -\beta^{2}\left(x+N^{\theta}-1\right)\left(y+N^{\theta}-1\right)\left(2 N^{\theta}+N-3\right) \\
& -\alpha \beta\left(x+N^{\theta}-1\right)\left(N-y+N^{\theta}-1\right)\left(2 N^{\theta}+N-3\right) \\
& -\alpha \beta\left(y+N^{\theta}-1\right)\left(N-x+N^{\theta}-1\right)\left(2 N^{\theta}+N-3\right) \\
& \left.-\alpha^{2}\left(N-x+N^{\theta}-1\right)\left(N-y+N^{\theta}-1\right)\left(2 N^{\theta}+N-3\right)\right] \\
& \quad\left[\left(2 N^{\theta}+N-2\right)^{2}\left(2 N^{\theta}+N-3\right)\right]^{-1} .
\end{aligned}
$$

Colocando $\alpha^{2}, \alpha \beta$ e $\beta^{2}$ em evidência, e simplificando os termos associados a cada uma dessas constantes temos

$$
\begin{aligned}
\langle\eta(x) ; \eta(y)\rangle_{\mu_{s s}}^{N-1}= & {\left[-\alpha^{2}\left(N-y+N^{\theta}-1\right)\left(x+N^{\theta}-1\right)\right.} \\
+ & 2 \alpha \beta\left(N-y+N^{\theta}-1\right)\left(x+N^{\theta}-1\right) \\
- & \left.\beta^{2}\left(N-y+N^{\theta}-1\right)\left(x+N^{\theta}-1\right)\right] \\
& {\left[\left(2 N^{\theta}+N-2\right)^{2}\left(2 N^{\theta}+N-3\right)\right]^{-1} } \\
= & -\frac{(\alpha-\beta)^{2}\left(x+N^{\theta}-1\right)\left(N-y+N^{\theta}-1\right)}{\left(2 N^{\theta}+N-2\right)^{2}\left(2 N^{\theta}+N-3\right)} .
\end{aligned}
$$

Seja agora $V=\left\{(x, y) \in\{0, \cdots, N\}^{2}: 0<x<y<N\right\}$, sua fronteira $\partial V=$ $\left\{(x, y) \in\{0, \cdots, N\}^{2}: x=0\right.$ ou $\left.y=N\right\}$ e $M=\left\{f: V \cup \partial V \rightarrow \mathbb{R} ;\left.f\right|_{\partial V}=0\right\}$.

Definição 6.2. O laplaciano discreto $\Delta_{V}^{N}: M \rightarrow \mathbb{R}$ é definido por

$\left(\Delta_{V}^{N} f\right)(x, y)=N^{2}\{f(x+1, y)+f(x-1, y)+f(x, y-1)+f(x, y+1)-$ $4 f(x, y)\}$, se $|x-y|>1$,

$\left(\Delta_{V}^{N} f\right)(x, x+1)=N^{2}\{f(x-1, x+1)+f(x, x+2)-2 f(x, x+1)$,

$e\left(\Delta_{V}^{N}\right)(x, y)=0$ se $(x, y) \in \partial V$.

Precisamos verificar que (6.21) é de fato a correlação para o SSEP em contato com reservatórios lentos. Inicialmente seja $\rho_{t}^{N}(x)=\left\langle\eta_{t}(x)\right\rangle$ como em (6.19), e $\varphi_{t}(x, y)=\left\langle\eta_{t}(x) ; \eta_{t}(y)\right\rangle$ como em (6.21). A ideia para a verificação é usar a técnica apresentada em [10], onde se mostra que a correlação do SSEP em contato com reservatórios lentos no caso $\theta=1$ satisfaz a equação discreta dada pela equação 8.3 de [10]. Fazendo algumas manipulações algébricas, a equação 8.3 de [10] para qualquer $\theta \geq 0$ é a dada por 


$$
\begin{cases}\partial_{s} \varphi_{s}(x, y)=\Delta_{V}^{N} \varphi_{s}(x, y)+g_{s}(x, y)+f_{s}(x, y), & \text { para }(x, y) \in V, \forall s \geq 0, \\ \varphi_{0}(x, y)=\left\langle\eta_{t}(x) ; \eta_{t}(y)\right\rangle, & \text { para }(x, y) \in V, \\ \varphi_{s}(x, y)=0, & \text { para }(x, y) \in \partial V, \forall s \geq 0,\end{cases}
$$

onde

$$
\begin{gathered}
g_{t}(x, y)+-\left(\nabla_{N}^{+} \rho_{t}^{N}(x)\right)^{2} \delta_{y=x+1}, \\
\nabla_{N}^{+} \rho_{t}^{N}(x)=N\left(\rho_{t}^{N}(x+1)-\rho_{t}^{N}(x)\right), \\
f_{s}(x, y)=\left(N^{2}-\frac{N^{2}}{N^{\theta}}\right) \varphi_{t}(x, y) \delta_{\{|y-x|=1, x=1, \text { ou } y=N-1\}},
\end{gathered}
$$

e não é difícil mostrar que a correlação $\varphi_{t}(x, y)$ que encontramos satisfaz (6.22). O leitor interessado pode verificar que a função obtida em (6.21) satisfaz a equação discreta (6.22).

Desta forma, concluímos que a expressão (6.21) é a correlação exata entre dois sítios quaisquer $x, y \in \Sigma_{N}$ para o SSEP em contato com reservatórios lentos. Este resultado sintetiza a grande versatilidade e eficiência do método da matriz ansatz para obter informações com respeito ao estado estacionário fora de equilíbrio de um sistema de partículas. Por exemplo, em [1] Lema 3.2 prova-se que

$$
\max _{0<x<y<N}\left|\varphi^{N}(x, y)\right| \leq \frac{C}{N^{\theta}+N}
$$

para provar o limite hidrostático, e em [10] esse resultado também é usado para provar as flutuações. Já com o resultado obtido acima, isso segue diretamente de (6.22). 


\section{Referências bibliográficas}

[1] BALdASSO, R.; MENEZES, O.; NEUMANN, A.; SOUZA, R. R. Exclusion process with slow boundary. Online, ArXiv, https://arxiv.org/abs/1407.7918 (2016).

[2] BERnARDIN, C.; GONÇALVES, P; OVIEDO, B. Slow to fast infinitely extended reservoirs for the symmetric exclusion process with long jumps. Online, ArXiv, https://arxiv.org/abs/1702.07216 (2017).

[3] BLYTHE, R. A.; EVANS, M. R. Non-equilibrium steady states of matrix-product form: a solver's guide. Journal of Physics: A Mathematical and Theoretical 40 (2007).

[4] BREZIS, H. Functional Analysis, Sobolev Spaces and Partial Differential Equations. Springer New York, 2010.

[5] DERRIDA, B. Non-equilibrium steady states: fluctuations and large deviations of the density and of the current. Journal of Statistical Mechanics Theory and Experiment (2007).

[6] DERRIDA, B.; EVANS, M. R.; HAKIM, V.; PASQUIER, V. Exact solution of a $1 \mathrm{~d}$ asymmetric exclusion model using a matrix formulation. Journal of Physics: A Mathematical and General Physics (1993).

[7] DERRIDA, B.; LEBOWITZ, J. L.; SPEER, E. R. Large deviation of the density profile in the steady state of the open symmetric simple exclusion process. Journal of Statistical Physics 107 (2002), 599-634.

[8] FRANCO, T.; GONÇALVES, P.; NEUMANN, A. Hydrodynamical behavior of symmetric exclusion with slow bonds. Ann. Inst. H. Poincaré Probab. Statist. 49, 2 (05 2013), 402-427.

[9] FRAnCO, T.; GONÇALVES, P.; NEUMANN, A. Phase transition of a heat equation with robin's boundary conditions and exclusion process. Transactions of the American Mathematical Society 367, 9 (2015), 61316158. 
[10] FRANCO, T.; GONÇALVES, P.; NEUMANN, A. Non-equilibrium and stationary fluctuations of a slowed boundary symmetric exclusion. Online, ArXiv, https://arxiv.org/abs/1608.04317 (2016).

[11] GONÇALVES, P.; LANDIM, C.; TONINELLI, C. Hydrodynamic limit for a particle system with degenerate rates. Annales de l'Institut Henri Poincaré: Probability and Statistics 45, 4 (2009), 887-909.

[12] HARRIS, T. E. Additive set-valued markov processes and graphical methods. The Annals of Probability (1978).

[13] LANDIM, C.; MILANES, A.; OLLA, S. Stationary and nonequilibrium fluctuations in boundary driven exclusion processes. Markov Processes and Related Fields 14, 2 (2008), 165-184.

[14] LAWRENCE C. EVANS. Partial Differential Equations: Second Edition, 2 ed. Graduate Studies in Mathematics. AMS, 2010.

[15] LEVIN D. A.; PERES Y., WILMER E. L. Markov Chains and Mixing Times. American Mathematical Society, 2007.

[16] LIGGETT, M. T. Stochastic Interacting Systems: Contact, Voter and Exclusion Processes, 1 ed. Springer-Verlag Berlin Heidelberg, 1999.

[17] LigGETT, M. T. Interacting Particle Systems. Springer, 2005.

[18] LIGGetT, M. T. Continuous Time Markov Processes. American Mathematical Society, 2010.

[19] SPOHN, H. Large Scale Dynamics of Interacting Particles. Theoretical and Mathematical Physics. Springer Berlin Heidelberg, 2011.

[20] VELÁZQUEZ, J. J. L. The porous medium equation: mathematical theory. Clarendon, 2007. 


\section{Semigrupos e geradores}

Abordaremos aqui apenas a parte da teoria de semigrupos que será utilizada na dissertação.

Assuma que $E$ é um espaço métrico e denote por $C(E)$ o conjunto das funções contínuas $f: E \rightarrow \mathbb{R}$, o qual é um espaço de Banach quando munido da norma $\|f\|_{\infty}=\sup _{\eta \in E}|f(\eta)|$. As funções $f$ podem ser vistas como observáveis, e estamos interessados em sua evolução temporal.

Seja $D[0, \infty)$ o espaço das funções definidas em $[0, \infty)$ com valores em $E$ contínuas à direita com limite à esquerda, isto é, $\xi:[0, \infty) \rightarrow E$ tal que $t \longmapsto \xi(t)$, com a propriedade $\xi(t)=\lim _{s \downarrow t} \xi(s)$ (contínua à direita) e $\xi\left(t^{-}\right)=\lim _{s \uparrow t} \xi(s)$ (limite à esquerda) existe. Chamaremos os conjuntos de funções com estas propriedades de funções càdlàg ${ }^{1}$. Para definir uma estrutura mensurável em $D[0, \infty)$, seja $\mathcal{F}$ a menor $\sigma$-álgebra em $D[0, \infty)$ tal que todas as funções $\eta$. $\eta_{s}$ para $s \geq 0$ são mensuráveis com respeito à $\mathcal{F}$. Se $\mathcal{F}_{t}$ é a menor $\sigma$-álgebra em $D[0, \infty)$ na qual todas as funções $\eta$. $\eta_{s}$ para $s \leq t$ são mensuráveis, então $\left\{\mathcal{F}_{t}\right\}_{t \geq 0}$ fornece uma filtração para o processo. $\mathrm{O}$ espaço filtrado $\left(D[0, \infty), \mathcal{F},\left\{\mathcal{F}_{t}\right\}_{t \geq 0}\right)$ fornece uma escolha genérica para espaço de probabilidade do processo estocástico, o qual pode ser definido como uma medida de probabilidade $\mathbb{P}$ em $D[0, \infty)$.

Definição A.1. Um processo de Markov em E é uma coleção $\left\{\mathbb{P}^{\zeta}\right\}_{\zeta \in E}$ de medidas de probabilidades em $D[0, \infty)$ com as seguintes propriedades:

a) $\mathbb{P}^{\zeta}\left(\eta . \in D[0, \infty): \eta_{0}=\zeta\right)=1$.

a) $\forall A \in \mathcal{F}$ a função $\zeta \longmapsto \mathbb{P}^{\zeta}(A)$ é mensurável em $E$.

c) $\mathbb{P}^{\zeta}\left(\tau_{t}^{-1}(A) \mid \mathcal{F}_{t}\right)(\eta)=\mathbb{P}^{\eta_{t}}(A)$ para toda $\zeta \in E, A \in \mathcal{F}$ e $t>0$, onde a função $\tau$ é a função translação definida na equação (3.4).

Definição A.2. Seja $\left\{\eta_{t}\right\}_{t \geq 0}$ um processo de Markov em E. Para cada $t \geq 0$ definimos o operador $S_{t}: C(E) \rightarrow C(E)$ por

$$
S_{t} f(\eta):=\mathbb{E}^{\eta} f\left(\eta_{t}\right),
$$

${ }^{1}$ do francês "continue à droite, limite à gauche". 
onde $\mathbb{E}$ é a esperança com respeito a medida do processo de Markov. Em geral $f \in C(E)$ não implica $S_{t} f \in C(E)$. Quando isso ocorre o processo é chamado processo de Feller.

Definição A.3. Seja $\left\{\eta_{t}\right\}_{t \geq 0}$ um processo de Feller em E. Então a família de operadores lineares $\left\{S_{t}\right\}_{t \geq 0}$ em $C(E)$ é um semigrupo de Markov, isto é

a) $S_{0}=I$, o operador identidade em $C(E)$.

b) A função $t \mapsto S_{t} f$ é contínua à direita para toda $f \in C(E)$.

c) $S_{t+s} f=S_{t} S_{s} f$ para toda $f \in C(E)$ e todo $s, t \geq 0$.

d) $S_{t} 1=1$ para todo $t \geq 0$.

e) $S_{t} f \geq 0$ para toda $f \in C(E)$ não negativa.

Os operadores $S_{t}$ determinam a evolução temporal dos observáveis. A importância do semigrupo de Markov está no fato que cada um corresponde a um processo de Markov, como é formulado no próximo teorema.

Teorema A.4. Suponha que $\left\{S_{t}\right\}_{t \geq 0}$ é um semigrupo de Markov em $C(E)$. Então, existe um único processo de Markov $\left\{\mathbb{P}^{\eta}, \eta \in E\right\}$ em E tal que

$$
S_{t} f(\eta)=\mathbb{E}^{\eta} f\left(\eta_{t}\right), \text { para toda } f \in C(E), \xi \in E \text {, e } t \geq 0 \text {. }
$$

Demonstração. Vide Teorema 1.1.5 de [17].

Definição A.5. Seja $\left\{S_{t}\right\}_{t>0}$ um semigrupo de Markov no espaço de Banach $C(E)$. Definimos $D(L):=\left\{f \in C(E): \exists \lim _{t \downarrow 0} \frac{S_{t} f-f}{t}\right\}$. O gerador infinitesimal do semigrupo $\left\{S_{t}\right\}_{t \geq 0}$ é definido como sendo o operador $L: D(L) \rightarrow E$ dado por

$$
L f:=\lim _{t \downarrow 0} \frac{S_{t} f-f}{t} .
$$

Teorema A.6. O operador L como definido acima é um gerador de Markov, isto é,

(a) $1 \in D(L)$ e $L 1=0$,

(b) para $f \in D(L), \lambda \geq 0: \min _{\xi \in X} f(\xi) \geq \min _{\xi \in X}(f-\lambda L f)(\xi)$,

(c) $D(L)$ é denso em $C(E)$ com respeito à norma $\|.\|_{\infty}$ e a imagem $\mathcal{R}(I d-$ $\lambda L)=C(E)$ para $\lambda>0$ suficientemente pequeno.

Demonstração. (a) é imediata de (A.3) e de $S_{t} 1=1$, os outros itens são bastante técnicos e podem ser encontrados na Seção 1.2 de [17]. 
Teorema A.7 (Hille-Yosida). Existe uma correspondência biunívoca entre geradores de Markov em $C(E)$ e semigrupos de Markov em $C(E)$. Essa correspondência é dada por (A.3) e por

$$
S_{t} f=e^{t L} f:=\lim _{n \rightarrow \infty}\left(I d-\frac{t}{n} L\right)^{-n} f \text { para } f \in C(E), t \geq 0 .
$$

Além disso, para $f \in D(L)$ e $S_{t} f \in D(L)$ e para todo $t \geq 0$,

$$
\frac{d}{d t} S_{t} f=S_{t} L f=L S_{t} f
$$

Demonstração. Para uma referência veja, por exemplo, [17], Teorema 1.2.9.

Lema A.8. Seja $\left\{\eta_{t}\right\}_{t \geq 0}$ um processo de Markov com semigrupo $\left\{S_{t}\right\}_{t \geq 0}$ e gerador infinitesimal L. Se fizermos uma mudança na escala de tempo por um fator $\theta(n)$, então o gerador infinitesimal para o processo $\left\{\eta_{t \theta(n)}\right\}_{t \geq 0}$ será dado por $\theta(n) L$.

Demonstração. Note da Definição A.5 que

$$
\theta(n) L f=\lim _{t \rightarrow 0} \theta(n) \frac{S_{t} f-f}{t} .
$$

Fazendo a mudança de variável $t=s \theta(n)$, temos

$$
\begin{aligned}
\theta(n) L f & =\lim _{t \rightarrow 0} \theta(n) \frac{S_{t} f-f}{t} \\
& =\lim _{s \rightarrow 0} \theta(n) \frac{S_{s \theta(n)} f-f}{s \theta(n)} \\
& =\lim _{s \rightarrow 0} \frac{S_{s \theta(n)} f-f}{s} \\
& =\tilde{L} f .
\end{aligned}
$$

Portanto, $\tilde{L}$ é o gerador para o processo $\left\{\eta_{t \theta(n)}\right\}_{t \geq 0}$, onde $\tilde{L}=\theta(n) L$. 
B

\section{Martingais e Processos de Markov}

Teorema B.1. Seja $\left\{X_{t}\right\}_{t \geq 0}$ um processo de Markov com gerador $L$ e com espaço de estados $E$ enumerável. Consideremos $F: \mathbb{R}^{+} \times E \rightarrow \mathbb{R}$ limitada, tal que:

(i) $\forall x \in E, F(\cdot, x) \in C^{2}\left(\mathbb{R}^{+}\right)$,

(ii) $\exists C<\infty$, tal que $\sup _{(s, x)}\left|\partial_{s}^{j} F(s, x)\right| \leq C$, para $j=1,2$.

Para $t \geq 0$, seja

$$
M_{t}^{F}:=F\left(t, X_{t}\right)-F\left(0, X_{0}\right)-\int_{0}^{t}\left(\partial_{s}+L\right) F\left(s, X_{s}\right) d s .
$$

Então, $\left\{M_{t}^{F}\right\}_{t \geq 0}$ é martingal com respeito à filtração natural do processo $\left\{X_{t}\right\}_{t \geq 0}$.

Demonstração. Vamos começar mostrando que $\left\{M_{t}^{F}\right\}_{t \geq 0}$ é um martingal com respeito à filtração natural do processo de Markov $\left\{X_{t}\right\}_{t \geq 0}$. Fixemos $0 \leq s \leq t$. Mostraremos que $\mathbb{E}_{x}\left[M_{t}^{F} \mid \mathcal{F}_{s}\right]=M_{s}^{F}$. Note que

$$
\begin{aligned}
\mathbb{E}_{x}\left[M_{t}^{F} \mid \mathcal{F}_{s}\right] & =\mathbb{E}_{x}\left[F\left(t, X_{t}\right)-F\left(0, X_{0}\right)-\int_{0}^{t}\left(\partial_{s}+L\right) F\left(s, X_{s}\right) d s \mid \mathcal{F}_{s}\right] \\
& =\mathbb{E}_{x}\left[F\left(t, X_{t}\right) \mid \mathcal{F}_{s}\right]-\mathbb{E}_{x}[\underbrace{F\left(0, X_{0}\right)}_{\mathcal{F}_{s}-\text { mensurável }} \mid \mathcal{F}_{s}] \\
& -\mathbb{E}_{x}\left[\int_{0}^{t}\left(\partial_{s}+L\right) F\left(s, X_{s}\right) d s \mid \mathcal{F}_{s}\right] \\
& =\mathbb{E}_{x}\left[F\left(t, X_{t}\right) \mid \mathcal{F}_{s}\right]-F\left(0, X_{0}\right)-\mathbb{E}_{x}\left[\int_{0}^{t}\left(\partial_{s}+L\right) F\left(s, X_{s}\right) d s \mid \mathcal{F}_{s}\right] \\
& =\mathbb{E}_{x}\left[F\left(t, X_{t}\right) \mid \mathcal{F}_{s}\right]-F\left(0, X_{0}\right)-\mathbb{E}_{x}\left[\int_{0}^{s}\left(\partial_{r}+L\right) F\left(r, X_{r}\right) d r\right. \\
& \left.+\int_{s}^{t}\left(\partial_{r}+L\right) F\left(r, X_{r}\right) d r \mid \mathcal{F}_{s}\right] \\
& =\mathbb{E}_{x}\left[F\left(t, X_{t}\right) \mid \mathcal{F}_{s}\right]-F\left(0, X_{0}\right)-\mathbb{E}_{x}[\underbrace{\left.\int_{0}^{s}\left(\partial_{r}+L\right) F\left(r, X_{r}\right) d r \mid \mathcal{F}_{s}\right]}_{\mathcal{F}_{s}-\text { mensurável }} \\
& +\mathbb{E}_{x}\left[\int_{s}^{t}\left(\partial_{r}+L\right) F\left(r, X_{r}\right) d r \mid \mathcal{F}_{s}\right] \\
& =\mathbb{E}_{x}\left[F\left(t, X_{t}\right) \mid \mathcal{F}_{s}\right]-F\left(0, X_{0}\right)-\int_{0}^{s}\left(\partial_{r}+L\right) F\left(r, X_{r}\right) d r \\
& -\mathbb{E}_{x}\left[\int_{s}^{t}\left(\partial_{r}+L\right) F\left(r, X_{r}\right) d r \mid \mathcal{F}_{s}\right] .
\end{aligned}
$$


Desta forma, pelo teorema de Fubini, mostrar que $\mathbb{E}_{x}\left[M_{t}^{F} \mid \mathcal{F}_{s}\right]=M_{s}^{F}$ é equivalente a mostrar que

$$
\begin{aligned}
\mathbb{E}_{x}\left[F\left(t, X_{t}\right) \mid \mathcal{F}_{s}\right] & =F\left(s, X_{s}\right)+\mathbb{E}_{x}\left[\int_{s}^{t}\left(\partial_{r}+L\right) F\left(r, X_{r}\right) d r \mid \mathcal{F}_{s}\right] \\
& =F\left(s, X_{s}\right)+\int_{s}^{t} \mathbb{E}_{x}\left[\left(\partial_{r}+L\right) F\left(r, X_{r}\right) \mid \mathcal{F}_{s}\right] d r .
\end{aligned}
$$

Para cada $r \geq 0$, denote por $F_{r}: E \rightarrow \mathbb{R}^{+}$(resp. por $F_{r}^{\prime}: E \rightarrow \mathbb{R}$ ) a função que em $x$ toma o valor $F(r, x)$ (resp. $\left(\partial_{r} F\right)(r, x)$ ). Da propriedade de Markov temos que

$$
\mathbb{E}_{x}\left[F\left(t, X_{t}\right) \mid \mathcal{F}_{s}\right]=\mathbb{E}_{X_{s}}\left[F_{t}\left(X_{t-s}\right)\right]=\left(S_{t-s} F_{t}\right)\left(X_{s}\right)
$$

Assim, da linearidade da esperança, da definição de semigrupo e fazendo uma mudança de variável, o segundo termo do lado direito de (B.1) é dado por

$$
\begin{aligned}
\int_{s}^{t} \mathbb{E}_{x}\left[\left(\partial_{r}+L\right) F\left(r, X_{r}\right) \mid \mathcal{F}_{s}\right] d r & =\int_{s}^{t}\left\{\mathbb{E}_{x}\left[\partial_{r} F\left(r, X_{r}\right) \mid \mathcal{F}_{s}\right]\right. \\
& \left.+\mathbb{E}_{x}\left[L F\left(r, X_{r}\right) \mid \mathcal{F}_{s}\right]\right\} d r \\
& =\int_{s}^{t}\left\{\mathbb{E}_{x}\left[F_{r}^{\prime}\left(X_{r}\right) \mid \mathcal{F}_{s}\right]+\mathbb{E}_{x}\left[L F\left(r, X_{r}\right) \mid \mathcal{F}_{s}\right]\right\} d r \\
& =\int_{s}^{t}\left\{\left(S_{r-s} F_{r}^{\prime}\right)\left(X_{s}\right)+\left(S_{r-s} L F_{r}\right)\left(X_{s}\right)\right\} d r \\
& =\int_{0}^{t-s}\left\{\left(S_{r} F_{s+r}^{\prime}\right)\left(X_{s}\right)+\left(S_{r} L F_{s+r}\right)\left(X_{s}\right)\right\} d r
\end{aligned}
$$

Logo, (B.1) reduz-se

$$
\left(S_{t-s} F_{t}\right)\left(X_{s}\right)=F_{s}\left(X_{s}\right)+\int_{0}^{t-s}\left\{\left(S_{r} F_{s+r}^{\prime}\right)\left(X_{s}\right)+\left(S_{r} L F_{s+r}\right)\left(X_{s}\right)\right\} d r
$$

Precisamos verificar a igualdade acima ponto a ponto no espaço amostral. Para isso vamos verificá-la substituindo $X_{s}$ por $x$, para cada $x \in E$. Como para $t=s$ a igualdade é satisfeita, precisamos verificar agora que a derivada de ambas expressões são iguais, isto é, que

$$
\partial_{t}\left(S_{t-s} F_{t}\right)(x)=\left(S_{t-s} F_{t}^{\prime}\right)(x)+\left(S_{t-s} L F_{t}\right)(x)
$$

para todo $x \in E$ e $0 \leq s<t$.

Para provar essa identidade vamos começar calculando o lado esquerdo da equação anterior, isto é, $\partial_{t}\left(S_{t-s} F_{t}\right)(x)$. Tomemos $h>0$ pequeno o suficiente para que nenhum dos índices seja negativo e reescrevemos a diferença $h^{-1}\left\{\left(S_{t-s+h} F_{t+h}\right)(x)-\left(S_{t-s} F_{t}\right)(x)\right\}$ como 


$$
\underbrace{h^{-1} \mathbb{E}_{x}\left[F_{t+h}\left(X_{t-s+h}\right)-F_{t}\left(X_{t-s+h}\right)\right]}_{=g_{1}}+\underbrace{h^{-1} \mathbb{E}_{x}\left[F_{t}\left(X_{t-s+h}\right)-F_{t}\left(X_{t-s}\right)\right]}_{=g_{2}} .
$$

Utilizando o teorema de Fubini e fazendo algumas manipulações algébricas, observe que podemos reescrever $g_{1}$ da seguinte maneira

$$
\begin{aligned}
g_{1} & =\frac{1}{h} \mathbb{E}_{x}\left[\int_{t}^{t+h} F_{r}^{\prime}\left(X_{t-s+h}\right) d r\right] \\
& =\frac{1}{h} \int_{t}^{t+h} \mathbb{E}_{x}\left[F_{r}^{\prime}\left(X_{t-s+h}\right)\right] d r \\
& =\frac{1}{h} \int_{t}^{t+h} \mathbb{E}_{x}\left[F_{r}^{\prime}\left(X_{t-s+h}\right)\right] d r \\
& +\mathbb{E}_{x}\left[F_{t}^{\prime}\left(X_{t-s+h}\right)-F_{t}^{\prime}\left(X_{t-s+h}\right)+F_{t}^{\prime}\left(X_{t-s}\right)-F_{t}^{\prime}\left(X_{t-s}\right)\right] \\
& =\frac{1}{h} \int_{t}^{t+h} \mathbb{E}_{x}\left[F_{r}^{\prime}\left(X_{t-s+h}\right)-F_{t}^{\prime}\left(X_{t-s+h}\right)\right] d r \\
& +\mathbb{E}_{x}\left[F_{t}^{\prime}\left(X_{t-s+h}\right)-F_{t}^{\prime}\left(X_{t-s}\right)\right]+\mathbb{E}_{x}\left[F_{t}^{\prime}\left(X_{t-s}\right)\right] .
\end{aligned}
$$

A hipótese $(i i)$ nos diz que $F$ tem derivadas de primeira e segunda ordens limitadas uniformemente na segunda coordenada, isso nos assegura que $F_{r}^{\prime}(x)$ é Lipschitz em $r$. Assim, existe $C \in \mathbb{R}$ tal que

$$
\left|F_{r}^{\prime}\left(X_{t-s+h}\right)-F_{t}^{\prime}\left(X_{t-s+h}\right)\right| \leq C(r-t)
$$

$\forall r, t \in \mathbb{R}^{+}$onde $t \leq r \leq h$. Logo,

$$
\begin{aligned}
0 & \leq\left|\frac{1}{h} \int_{t}^{t+h} \mathbb{E}_{x}\left[F_{r}^{\prime}\left(X_{t-s+h}\right)-F_{t}^{\prime}\left(X_{t-s+h}\right)\right] d r\right| \\
& \leq \frac{1}{h} \int_{t}^{t+h} \mathbb{E}_{x}\left[\left|F_{r}^{\prime}\left(X_{t-s+h}\right)-F_{t}^{\prime}\left(X_{t-s+h}\right)\right|\right] d r \\
& \leq \frac{1}{h} \int_{t}^{t+h} \mathbb{E}_{x}[C(r-t)] d r=\frac{C}{h} \int_{t}^{t+h}(r-t) d r=\frac{C h}{2} \longrightarrow 0
\end{aligned}
$$

quando $h \downarrow$. Consequentemente o primeiro termo de (B.3) vai para zero quando $h \downarrow 0$. O segundo termo de (B.3) pode ser reescrito como $h^{-1}\left(\left(S_{t-s+h} F_{t}^{\prime}\right)(x)-\left(S_{t-s} F_{t}^{\prime}\right)(x)\right)$, e também vai para zero quando $h \downarrow 0$ pois o semigrupo $S_{t}$ é contínuo. Portanto, quando $h \downarrow 0$, a expressão $g_{1}$ em (B.3) converge para $\mathbb{E}_{x}\left[F_{t}^{\prime}\left(X_{t-s}\right)\right]=\left(S_{t-s} F_{t}^{\prime}\right)(x)$. Vamos analisar agora a expressão $g_{2}$ de (B.2). De fato,

$$
\begin{aligned}
\mathbb{E}_{x}\left[\frac{F_{t}\left(X_{t-s+h}\right)-F_{t}\left(X_{t-s}\right)}{h}\right] & =\frac{1}{h} \int_{t-s}^{t-s+h} \mathbb{E}_{x}\left[\frac{d}{d r} F_{t}\left(X_{r}\right)\right] d r \\
& =\frac{1}{h} \int_{t-s}^{t-s+h} \mathbb{E}_{x}\left[\left(\partial_{r} F_{t}\right)\left(X_{r}\right)\right] d r \\
& =\frac{1}{h} \int_{t-s}^{t-s+h} \frac{d}{d r}\left(S_{r} F_{t}\right)(x) d r
\end{aligned}
$$




$$
=\frac{1}{h}\left[\left(S_{t-s+h}\right)(x)-\left(S_{t-s} F_{t}\right)(x)\right] .
$$

Tomando o limite na expressão acima e utilizando o Teorema de Hille-Yosida (A.7), temos

$$
\begin{aligned}
\lim _{h \rightarrow 0} \frac{\left(S_{t-s+h} F_{t}\right)(x)-\left(S_{t-s} F_{t}\right)(x)}{h} & =\frac{d}{d(t-s)}\left(S_{t-s} F_{t}\right)(x) \\
& =\left(L S_{t-s} F_{t}\right)(x)=\left(S_{t-s} L F_{t}\right)(x) .
\end{aligned}
$$

Portanto,

$$
\partial_{t}\left(S_{t-s} F_{t}\right)(x)=\left(S_{t-s} F_{t}^{\prime}\right)(x)+\left(S_{t-s} L F_{t}\right)(x)
$$

para $0 \leq s \leq t$, como queríamos mostrar. 


\section{C \\ Gerando um processo de Poisson}

Proposição C.1 (Método da Transformada Inversa). Sejam $F: \mathbb{R} \rightarrow$ $[0,1]$ a função de distribuição de uma variável aleatória e $F^{-}$a sua inversa generalizada, dada por $F^{-}(t)=\inf \{x \in \mathbb{R}: t \leq F(x)\}$. Se $U$ é uma variável aleatória que segue uma lei uniforme no intervalo $(0,1)$, então $F$ é a função de distribuição da variável aleatória $F^{-}(U)$.

\section{Gerando uma variável aleatória com distribuição exponencial}

Se $X$ é uma variável aleatória com distribuição exponencial com taxa 1, então sua função de distribuição é dada por $F(x)=1-e^{-x}, x \geq 0$. Como $0 \leq F(x) \leq 1$, tomando $F(x)=U$, onde $U$ tem lei uniforme no intervalo $(0,1)$ temos $U=F(x)=1-e^{-x}$. Então, aplicando o logaritmo temos $x=\ln (1-U)$. Daí, pode-se gerar uma variável aleatória exponencial com parâmetro 1 gerando um número aleatório $U$ e em seguida fazendo $X=F^{-1}(U)=-\ln (1-U)$. Uma pequena economia de tempo também pode ser obtida notando que $1-U$ também é uniforme em $(0,1)$ e assim, $-\ln (1-U)$ tem a mesma distribuição que $-\ln (U)$.

Além disso, note que se $X$ é uma exponencial com média 1, então para qualquer constante $c, c X$ é uma variável aleatória com média $c$. Logo, uma variável aleatória exponencial $X$ de parâmetro $\lambda$ pode ser gerada através da geração de um número aleatório $U$ e fazendo $X=-\frac{1}{\lambda} \ln (U)$.

\section{Gerando de um processo de Poisson}

Suponha que se quer gerar os primeiros $n$ tempos dos eventos de um processo de Poisson com taxa $\lambda$. Para isso, lembremos que os tempos entre os eventos sucessivos de tal processo são variáveis aleatórias exponenciais independentes, cada uma com taxa $\lambda$. Assim, uma maneira de gerar o processo consiste em gerar os tempos entre os eventos. Se forem gerados $n$ números aleatórios $U_{1}, U_{2}, \ldots, U_{n}$ e calculados $X_{i}=-\frac{1}{\lambda} \ln U_{i}$, então $X_{i}$ pode ser considerado como o tempo entre o $(i-1)$-ésimo e o $i$-ésimo evento do processo de Poisson. Uma vez que o tempo atual do $j$-ésimo evento será igual à soma 
dos primeiros $j$ intervalos de tempo entre os eventos, segue-se que os valores gerados dos primeiros $n$ tempos dos eventos são $\sum_{i=1}^{j} X_{i}, j=1, \ldots, n$. Assim, para gerar as primeiras $T$ unidades de tempo do processo de Poisson, podese seguir o procedimento anterior de gerar sucessivamente os tempos entre os eventos, parando quando a soma ultrapassar $T$.

O algoritmo seguinte pode ser usado para gerar todos os tempos dos eventos que ocorrem no intervalo $[0, T]$ de um processo de Poisson com taxa $\lambda$. No algoritmo, $t$ refere-se ao tempo, $I$ é o número de eventos que ocorreram no tempo $t$, e $S(I)$ é o tempo do evento mais recente.

\section{Gerando as primeiras $T$ unidades de tempo de um Processo de} Poisson com taxa $\lambda$.

Passo 1. $t=0, I=0$.

Passo 2. Gere um número aleatório $U$.

Passo 3. $t=t-\frac{1}{\lambda} \ln U$. Se $t>T$, pare.

Passo 4. $I=I+1, S(I)=t$.

Passo 5. Vá para o passo 2.

O valor final de $I$ no algoritmo acima vai representar o número de eventos que ocorrem no tempo $T$, e os valores $S(1), \ldots, S(I)$ serão os tempos dos $I$ eventos em ordem crescente. 